\title{
Improving the limits of detection in sensor systems
}

J.F. van der Bent 


\section{Thesis committee}

\section{Promotor}

Prof. Dr C.J.M. van Rijn

Special Professor Microsystem and NanoTechnology for Agrofood and Health

Wageningen University \& Research

\section{Other members}

Prof. Dr W.G. van der Wiel, University of Twente, Enschede

Prof. Dr E.J. Woltering, Wageningen University \& Research

Dr L.J.M. van Moergestel, HU University of Applied Sciences Utrecht

Dr M. Tichem, Delft University of Technology

This research was conducted under the auspices of the Graduate School VLAG (Advanced studies in Food Technology, Agrobiotechnology, Nutrition and Health Sciences). 


\title{
Improving the limits of detection in sensor systems
}

\author{
J.F. van der Bent
}

\section{Thesis}

submitted in fulfilment of the requirements for the degree of doctor

at Wageningen University

by the authority of the Rector Magnificus,

Prof. Dr A.P.J. Mol,

in the presence of the

Thesis Committee appointed by the Academic Board

to be defended in public

on Monday 10 April 2017

at 4 p.m. in the Aula. 
J.F. van der Bent

Improving the limits of detection in sensor systems,

127 pages.

PhD thesis, Wageningen University, Wageningen, the Netherlands (2017)

With references, and summary in English

ISBN: 978-94-6343-055-5

DOI: http://dx.doi.org/10.18174/401509 


\section{Table of contents}

$\begin{array}{lll}\text { Chapter } 1 \quad \text { General Introduction } & 6\end{array}$

Chapter 2 Defining the Signal Measurement System

21

Chapter 3 Temperature Balanced Hydrogen Sensor System with

$\begin{array}{ll}\text { Coupled Palladium Nanowires } & 37\end{array}$

Chapter $4 \quad$ Improving the Limits of Detection in Potentiometric Sensors 57

Chapter 5 Improving the Limits of Detection in Capacitive Sensor Systems 71

Chapter $6 \quad$ General Discussion $\quad 91$

$\begin{array}{ll}\text { References } & 97\end{array}$

$\begin{array}{ll}\text { Appendix A } & 103\end{array}$

$\begin{array}{ll}\text { Appendix B } & 114\end{array}$

$\begin{array}{ll}\text { Summary } & 116\end{array}$

$\begin{array}{ll}\text { Acknowledgements } & 119\end{array}$

$\begin{array}{ll}\text { About the Author } & 121\end{array}$

$\begin{array}{ll}\text { List of Publications } & 123\end{array}$

$\begin{array}{ll}\text { Overview of Completed Training Activities } & 125\end{array}$ 


\section{Chapter 1}

\section{General Introduction}

A sensor's main task is to detect the occurrence of an event or presence of a target in a given environment and to provide a signal as a measure. This signal must be converted using a suitable signal adaptation method to produce a specific output. For example, in the field of chemical sensing, electrical or optical signals are typically processed and converted into a concentration of the target. The performance of sensors is generally characterized by sensitivity, selectivity, limit of detection, stability, power efficiency and response time. These performance criteria are explained in this first chapter and are then used as focal points in chapters 3, 4 and 5. Power efficiency is also briefly discussed in these chapters, while chapter 1 concentrates on the current developments from the analogue wired domain to the digital and wireless domain in the sensor landscape.

A sensor's sensitivity indicates how much its output changes in response to a change in the concentration of the target agents being measured. The selectivity of a sensor indicates the cross-sensitivity to agents other than the target. The higher the selectivity, the lower the cross-sensitivity. Sensor stability is indicated by several factors. A perfectly stable sensor would have a steady output level over time with unchanged test parameters, no drift of the baseline and no decay of the active material of the sensor over time. The response time and the limit of detection are strongly interrelated. An analogue signal response needs to be distinguished from noise in order to measure the response time after exposure.

The limits of detection of sensors are generally determined by several performance criteria, which may be interrelated. The noise floor of prototype sensors, for example, is not known beforehand, nor are the cross-sensitivities to other factors such as a changing environment. This means that a test environment is needed. The conceptualization, design and verification of a signal measurement system (SMS) are crucial steps in sensor research. Sensitivity to other variables than the detection agent or analyte (cf. selectivity) is often problematic and can only be countered when cross-sensitivity properties are properly charted. This means that, apart from developing the correct 
signal processor (cf. interfacing), the properties of the signal measurement system (cf. chamber) must also be selected, defined and verified.

In the last decade nanotechnology has enabled scientists to develop small nano-sensors of fair to good quality operating at reduced power consumption levels with, in many cases, improved sensitivity. Furthermore, with the maturing and downscaling of available micro-controller units (MCU's) and the improved power usage of these devices, low-power digital signal techniques can be integrated in the sensor device itself, improving its properties. This thesis focusses on exploring and designing the proper signal measurement system for these sensors and proposes different approaches to improving the limit of detection (LoD) (Figure 1).

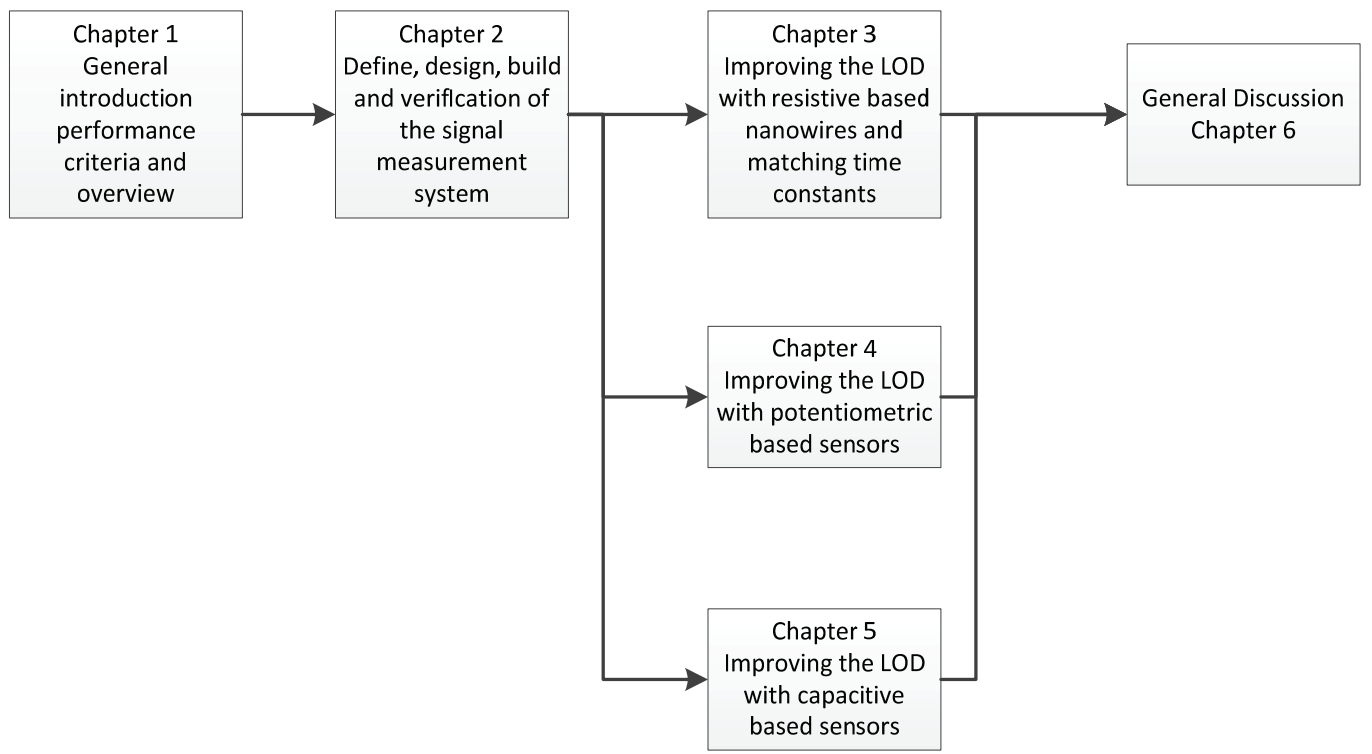

Figure 1. Schematic overview of the thesis structure

After the introduction in chapter 1, the signal measurement system is described in chapter 2. Here the different possibilities for testing and the description of the experimental setup are also presented in detail. Chapters 3, 4 and 5 address improvements in the limits of detection of various (micro- and nano-)sensors with different design solutions for the signal measurement systems. The discussion in chapter 6 concludes this thesis and connects all findings with a view towards future work and developments. Table 1 shows the performance criteria examined in each of the chapters. 
Table 1. Performance criteria and their global subdivision in the chapters

\begin{tabular}{lccccc} 
performance critera & chapter 1 & chapter 2 & chapter 3 & chapter 4 & chapter 5 \\
\hline sensitivity & $\mathrm{x}$ & $\mathrm{x}$ & & $\mathrm{x}$ \\
\hline selectivity & $\mathrm{x}$ & $\mathrm{x}$ & $\mathrm{x}$ & \\
\hline limit of detection & $\mathrm{x}$ & $\mathrm{x}$ & $\mathrm{x}$ & $\mathrm{x}$ \\
\hline stability & $\mathrm{x}$ & & $\mathrm{x}$ & $\mathrm{x}$ \\
\hline response time & $\mathrm{x}$ & $\mathrm{x}$ & & \\
\hline power efficiency & $\mathrm{x}$ & & $\mathrm{x}$ & $\mathrm{x}$ & \\
\hline
\end{tabular}

\subsection{Defining the LoD}

Although LoD is a term widely used in many disciplines, the definition can vary greatly. In chemistry, for instance, the definition of LoD used in spectral analysis is well established (Shrivastava \& Gupta, 2011) as:

$\mathrm{LoD}=\mathrm{X}_{\mathrm{b} 1}+3 \mathrm{~S}_{\mathrm{b} 1}$

Where $\mathrm{X}_{\mathrm{b} 1}$ is the mean concentration of a zero concentration sample and $\mathrm{S}_{\mathrm{b} 1}$ is the standard deviation from this blank. The weakness in this approach is the same throughout the various fields that use it. Somehow one has to prove what the minimal amount of analyte, or signal, would be to obtain the base level equivalent to the noise. Other methods include a more intuitive visual definition or a hybrid of the two as used in this work. Figure 2 shows signal with a significant measurable signal relative to the noise level where the signal to noise ratio $=2$.signal / noise: 


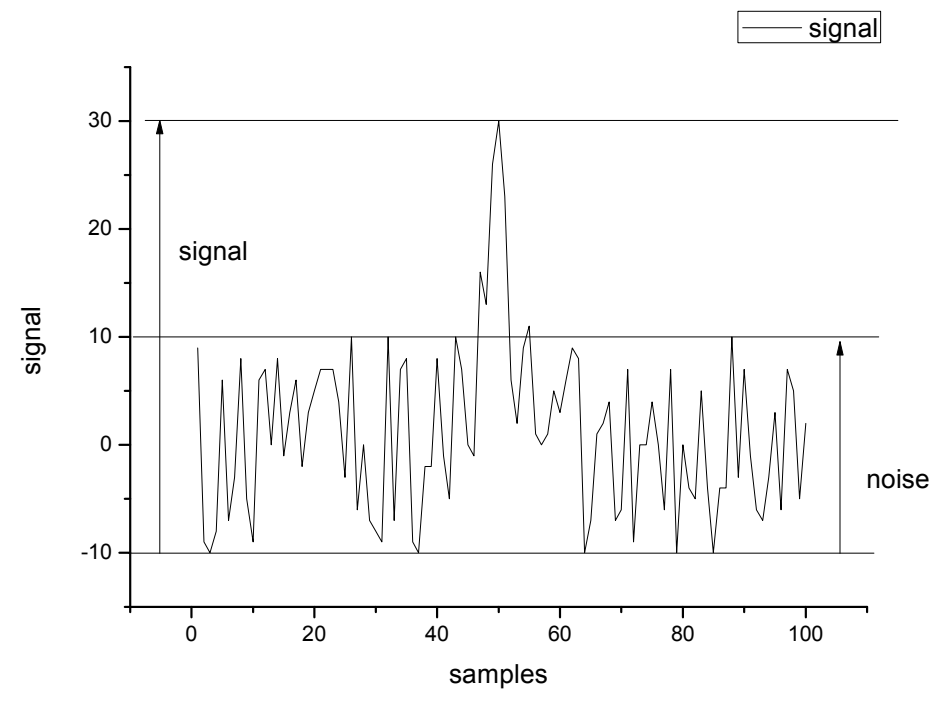

Figure 2. Relationship between signal to noise ratio and LoD

The usefulness of this data depends heavily on what is on the $\mathrm{x}$-axis. When the frequency domain is on the $\mathrm{x}$-axis, the data is more valid than when the time domain is on the $\mathrm{x}$ axis. There are many reasons for this. When, for instance, the cross-sensitivity of a sensor being tested is greater than the signal towards the agent, the limit of detection will be determined by the cross-sensitivity. When the drift of a sensor is greater than the signal, the detection limit will be determined by the amplitude of the drift. This means that, in order to gain insight into the properties of a sensor, it is vital that its crosssensitivities be properly measured. After these properties are known, counter-actions can be engineered.

\subsection{Sensing with Reduced Power Levels}

An aim throughout this thesis, focussed by their end application, is the ability to increase the limit of detection at reduced power levels. This may be accomplished by maintaining the active material of the sensor at room temperature or by designing a low-power signal measurement system. In this way sensing can be performed at a low power level for battery operated nodes (cf. wireless sensor network, WSN). For example, in the case of $\mathrm{CO}_{2}$ sensing, nearly all available commercial sensors operate with a preheated active sensor layer that consumes a relatively large amount of power (Harbeck, Szatvanyi, Barsan, Weimar, \& Hoffmann, 2003; Satyanarayana, Noh, Lee, Jin, \& Park, 2009). The 
advantage of a high operating temperature is that it makes the sensor less crosssensitive to humidity. However, it takes time before the sensor is thermally and electrically stabilized and ready to measure within specifications. Power usage can normally be lowered by duty cycling. However, when the active material needs to be preheated, the stabilization time of the sensor after power up can be relatively long, even up to hours.

During the stabilization period, power is drawn from the supply, i.e. a battery, and measurements cannot be performed. The well-known TGS4161 $\mathrm{CO}_{2}$ detector (Figaro USA, 2005), for instance, known for its stability and long term usage, draws $250 \mathrm{~mW}$ for the heater alone and needs at least one hour for stabilization. When the sensor is to be used in a WSN, $250 \mathrm{~mW}$ is not preferred, and recent work (Fadel et al., 2015; Mladen, 2007) shows that $100 \mu \mathrm{W}-250 \mu \mathrm{W}$ is more appropriate, depending on the application. Chapter 4 includes discussion of a $\mathrm{CO}_{2}$ sensor that is able to detect $\mathrm{CO}_{2}$ at room temperature without the need for a heating element in the sensor. With the use of digital signal processing, cross-sensitivity can also be further reduced. In Chapter 3, another cross-sensitivity reduction effect is proposed with a focus on hydrogen detection, including special attention to early detection in the event of a hydrogen leak. Here, digital signal processing is used to compensate for temperature fluctuations, although the modus operandi differs from the $\mathrm{CO}_{2}$ method.

Chapter 5 uses a capacitive sensing principle that is, apart from cross-sensitivity for temperature, also sensitive to humidity changes. Temperature compensation was also used in chapter 3, although here a more direct approach was taken by using the temperature response from one nanowire to compensate for another.

When new data is collected from an experimental sensor, data analysis can then be performed. This analysis may result, for instance, in filtering or/and linearization or compensation with temperature readings or humidity readings. These steps are performed after the data is collected and the calculations are done off-line. When off-line calculations are used during analysis of the data, power usage is no real issue. When battery-operated sensors are used in WSN, the power needed for on-line linearization algorithms, cross-sensitivity compensation and transmission of data, becomes an issue. Integration of sensor and compensation electronics together with a wireless transceiver (see figure 3) forms the basis for compensation, creating a smart sensor, with calculations needing to be performed on-line while collecting data. 


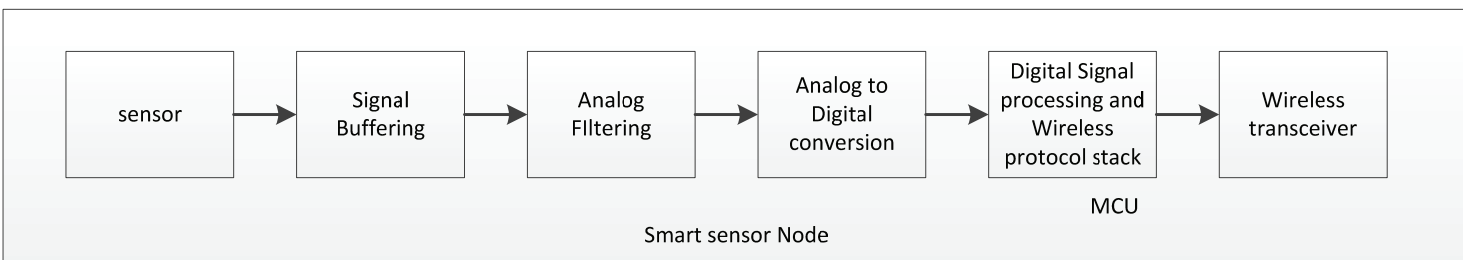

Figure 3. Simplified smart sensor node setup

Micro Computer Units (MCU), also known as microcontrollers, can currently be classified into three groups: low-end, mostly 8 bit processors; mid-range, mostly 16 bit processors; and high-end, mostly 32 bit processors. The common denominator of all these processors is that, in addition to the processing core itself, they incorporate random access memory (RAM) to hold program variables, electronically erasable programmable read only memory (EEPROM) or flash memory to store the program, and peripheral host devices such as serial interface controllers (RS232, SPI, $\mathrm{I}^{2} \mathrm{C}$ ), general purpose input/output pin controllers, and timers.

From the perspective of power usage, interfacing of sensors with the help of a low-end MCU has greatly improved over the last decade. Semiconductor microcontrollers are available in smaller packages. The 8051 microcontroller, for example, was only available in a DIP40 (53x16mm) package when it was launched in 1981, while comparable microcontrollers now come in ball grid (BGA) packages $(5 \times 5 \mathrm{~mm})$ using only a fraction of the space needed on a printed circuit board in comparison to the 8051 .

When comparing technology from, for example, 2006 to that of 2014 with regard to the power usage of the processing core, there is an improvement of over 50 times (Microchip PIC16F628 @ 4MHz, 8 bit 10 mW in 2006; Microchip PIC16LF1454 4MHz, 8bit, $180 \mu \mathrm{W}$ from 2014). This phenomenon is observed in the entire range of lowpower MCUs. Furthermore, most low-end MCUs have a wide array of ultra-low power modes, clearing the road for intricate duty cycling of its power (see figure 4). 


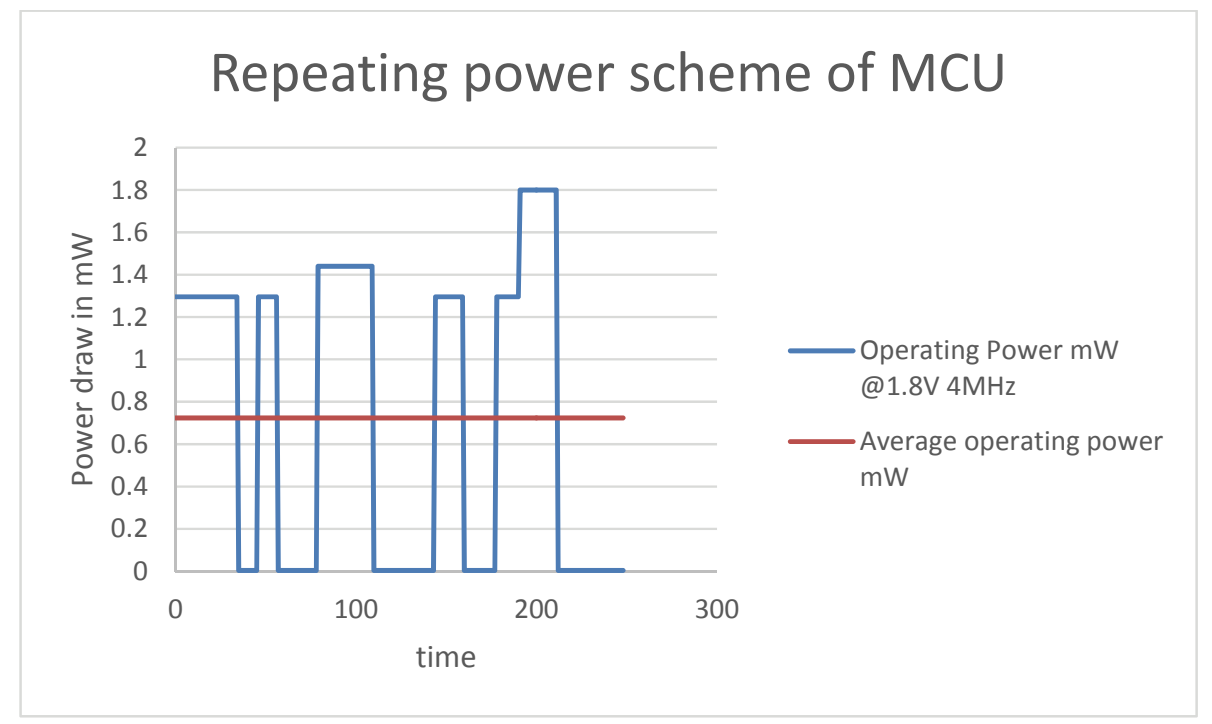

Figure 4. Power scheme of an MCU using different power modes

Most semiconductor vendors have support tools for these low-power modes in order to derive an estimation of the total power usage of the device. Unlike with sensors which need a fairly long stabilization time, the switching of an MCU from one power mode to another takes only a few processor cycles. Many operational amplifiers and analogue-todigital converters also have shutdown modes where the total current drawn from the supply drops to or below $1 \mu \mathrm{A}$, contributing to an extension of the battery life during low-power sleep modes. This means that a low-power sensor can meet the demand with the addition of low power MCUs, enabling the device for digital signal processing and incorporating the sensor in a WSN.

In conclusion, the idea of duty cycling has been welcomed and can be used to extend battery life if the stabilization time of the device that is switched on/off is less than the measurement interval. Furthermore, over the last decade the power consumption of MCUs in general, and low-end ones in particular, has greatly improved and their physical size has been dramatically reduced.

\subsection{The Added Advantage of an MCU in a WNS}

Over the last decade, many semiconductor companies have developed their own wireless transfer protocols. Texas Instruments, for example, has developed the ANT protocol, while Nordic Inc. has developed their so called 'shock burst' protocol. Although 
all of these are embedded in software and hardware based on the general open system interconnection (OSI) layer model, see figure 5, very few of them are interoperable.

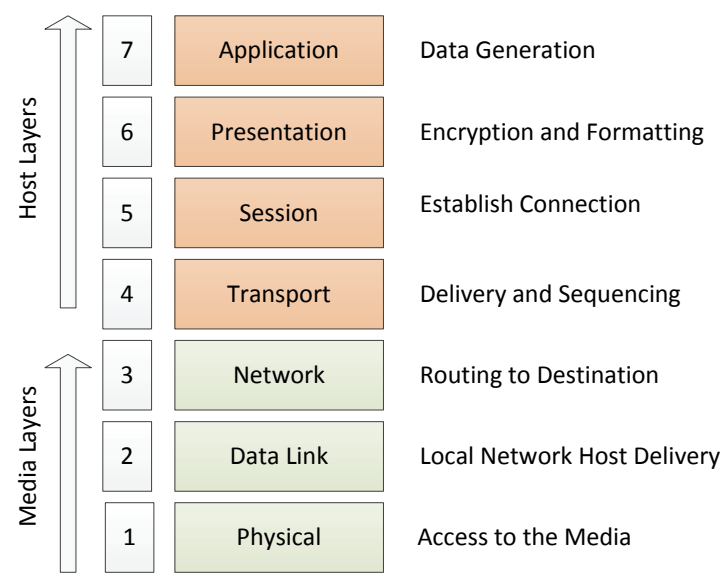

Figure 5. General OSI model layer presentation

Although the physical layer uses one of the same open frequency spaces $(2.4 \mathrm{GHz}, 868$ $\mathrm{MHz}, 433 \mathrm{MHz}$ in Europe), the different protocols cannot be integrated with each other. In a sense, all available protocols and methods try to perform the same task of sending data from A to B with the guarantee of data reaching the destination or notification of failure being sent, and do so with a limited amount of energy usage during the process. They differ in whether or not a mesh structure, a technique to transport data over multiple nodes, can be used and what part of the functionality based on the OSI model is placed in hardware (see figure 5). A larger stack, from the perspective of bytes used, comes with features such as data encryption and mesh capability such as Zigbee and LORA (long range low power), while a smaller stack only supports peer to peer (P2P) data transport from one node to another. In order to gain a larger market share, several consortia have been created. The larger ones, the Zigbee alliance and the LORA alliance for instance, have united many semiconductor companies to improve inter-operability in devices within the same domain.

Apart from the MCU task of running a certain software stack such as Zigbee in order to transfer wireless data from a node to a receiver, there is another advantage of having an MCU present. There is an ongoing reduction of the power needed to operate a MCU. With regard to the development of WSN transceiver chips during the last decade, there has 
been a reduction of the power draw with more efficient methods for wireless transport of data in a shorter period of time and also with the use of more sensitive highfrequency frontends. The bottleneck in these wireless developments remains the physical air path. In order to transfer information from point A to point $B$, a minimum of power is still needed. Literature (Fadel et al., 2015; Wang, Lu, Narayanan, Redmond, \& Lovell, 2015) shows that a transmitter and a receiver still need at least $17 \mathrm{~mW} @ 3.3 \mathrm{~V}$, nRF52832, to operate at a short distance of less than 10 metres in an open field. This means that sending information over the air places a significant demand on the power budget. The signal processing needed for analysis of the signal from the sensor and adequate compensation for cross-sensitivity and linearizing is, from the perspective of the power budget, more effectively handled on the sensor node itself.

\subsection{DSP and Typical MCU Sensor Tasks Used in This Work}

Digital signal processing can be performed with every available MCU that has a time slice remaining. The digital signal processor, however, was designed to effectively handle DSP functions. At the core of these DSP functions, such as DFT (digital Fourier transformation) of a FIR (finite impulse response) filters, is the multiply and accumulate function. Two data values are multiplied and added to another value. Because this core routine is executed constantly, the von Neumann-based architectures have their bottleneck in the address bus and the data-bus of the MCU system, because they need to be accessed three times for every multiply and accumulate cycle. The digital signal processor solves this problem by adding an extra data and address bus connected to a different memory. This means that the digital signal processor is best equipped for the job, but all microcontrollers can execute the algorithms. The reason that they can be used in combination with sensors is that the majority of the sensors do not have a large data throughput. This is of course related to the sampling frequency of the converters, which, for a temperature sensor, for example, is less than once every 10 seconds.

Signal processing needed in sensor nodes ranges from linearization to filtering techniques. Linearization of a response means that the signals from both experimental sensors and those used in industry exhibit a deviation from an ideal relationship between the detection response and the actual measure. In this example, a signal was recorded along with a reference signal from a calibrated humidity sensor. When the 
response data from the prototype sensor is plotted on one axis in a graph, and the response of the calibrated sensor on the other axis, a relation can be found by fitting. Ideally, the fitted response has a standard deviation, denoted as $\sigma^{2}$ or $\mathrm{R}^{2}$, of one, indicating a perfect fit. When the standard deviation is close to 1 and the maximum deviation from the reference is considered to be within specifications, this relation can be used for linearization. Figure 6 shows an example of the linearization of a Figaro TGS822 acetone sensor for humidity changes.

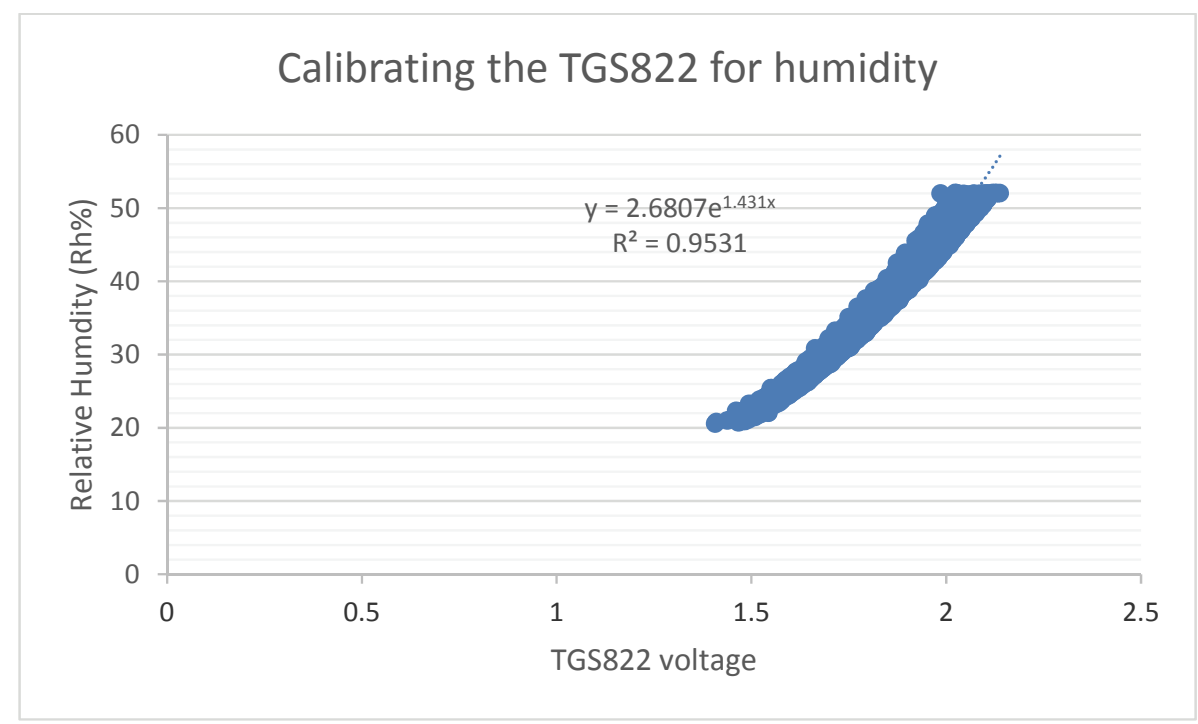

Figure 6. The linearization graph of a TGS822 for humidity

In figure 6, an exponential relationship was found between the voltage signal produced by the TGS822 and the relative humidity. During this measurement the temperature was kept stable at $25.06^{\circ} \mathrm{C} \pm 0.07^{\circ} \mathrm{C}$ to ensure that only the humidity cross-sensitivity was addressed. The exponential correction of $2.6807 \mathrm{e}^{1.431 \mathrm{x}}$ with a standard deviation of 0.95 was calculated using the fitting function of Excel. The task of the MCU in the system is now restricted to a minor modification of the recoded sensor data to the linearization formula.

If the standard deviation is outside the specified boundaries and an acceptable fit cannot be found, the data can be used by compiling a specific reference lookup table (see figure 7). 


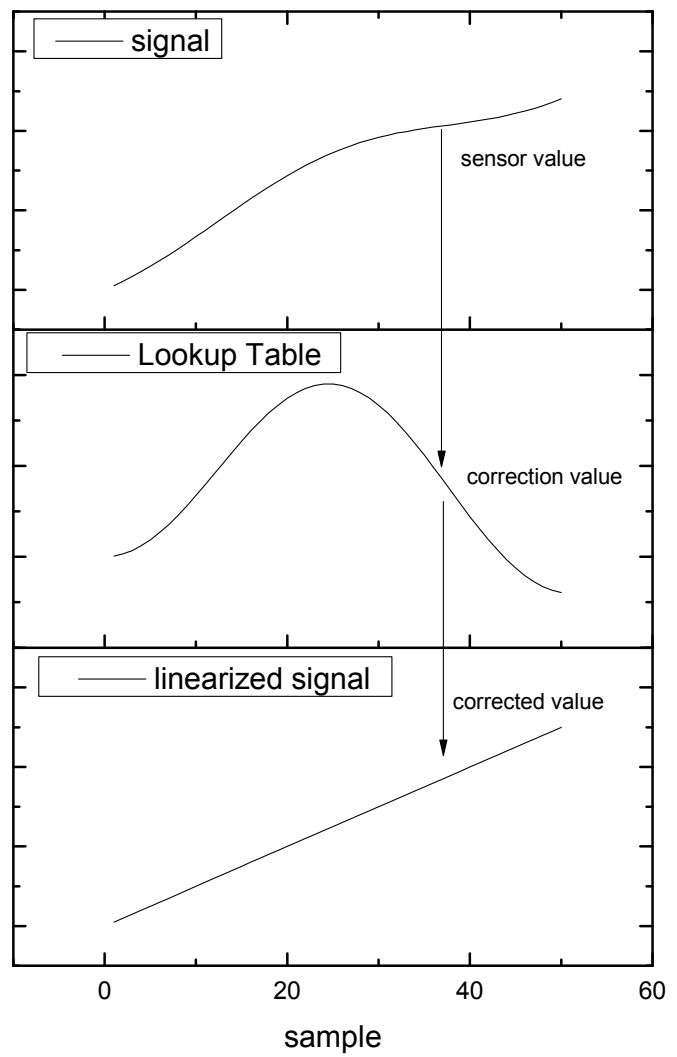

Figure 7. Linearization example of a sensor signal

The top graph of figure 7 shows the input signal of the sensor that is to be linearized with the middle graph in order to produce the linearized signal output in the bottom graph.

Although the signal can be linearized with a lookup table (LUT), and MCUs are well equipped to handle this task, fitting the signal to a linear, polynomic or exponential equation is preferred. Fitting in general means that the response of the sensor can be corrected in a well-defined manner, as shown in Chapter 4 Section 2.2, while an LUT has the danger of capturing a one-time erratic sensor behaviour and the size of the LUT defines the resolution. When a value from the sensor is in between two data points of the LUT, an estimation must be made using linear or higher order interpolation. Filtering of a signal has advantages when, for instance, a low pass filter is used to remove spikes, or undesired high frequency, in a signal. In the case of the $\mathrm{CO}_{2}$ sensor, a single spike may trigger an exception in the MCU. 
This filtering, for example a moving average filter (MAF), must be performed in combination with analogue filtering electronics in order to avoid folding of the signal to the base band (Smith, 1997). The advantage of signal processing in the sensor node itself, which defines the node as intelligent, is that not only can filtering be performed but linearization and properties such as temperature dependence can also be handled more efficiently with respect to the power budget.

\subsection{Capturing a Sensor's Behaviour}

Once a sensor is selected or prepared, it is placed in a controlled environment where parameters such as humidity, temperature and the agent gas that is to be detected can be changed. Chapter 2 describes the functionality and the construction of the signal measurement system used for the work presented in Chapters 3,4 and 5 . Before the behaviour of a sensor can be analysed, its basic characteristics must be fully understood. This means that, for instance, in the case of $\mathrm{CO}_{2}$ detection as described in chapter 4 , the voltage output of the potentiometric sensor needs to be buffered. The input impedance of the operational amplifiers must be at least $10 \mathrm{G} \Omega$. A lower input impedance would draw too much bias current from the sensor, resulting in a lowered response and unstable baseline signal. A functional hypothesis can then be created by placing the sensor in the chamber and performing initial measurements. In the case of $\mathrm{CO}_{2}$ sensing, a high correlation between the two sensors for temperature variations could be observed when two sensors where placed in the chamber while performing simultaneous measurements with the same technique. After confirming the correlation, a differential interface was designed on the basis of an instrumental amplifier in order to collect data and to minimize the error in the analogue-to-digital converter phase. The reason for using an instrumentation amplifier is that the unwanted common mode voltage, equally present at both inputs, is only amplified one time and subtracted from itself in the final stage. The differential or wanted voltage is amplified both in the first and the final stage accordingly, as used in medical signal detection (Metting van Rijn, Peper, \& Grimbergen, 1990). Figure 8 shows a simplified model of this approach. 


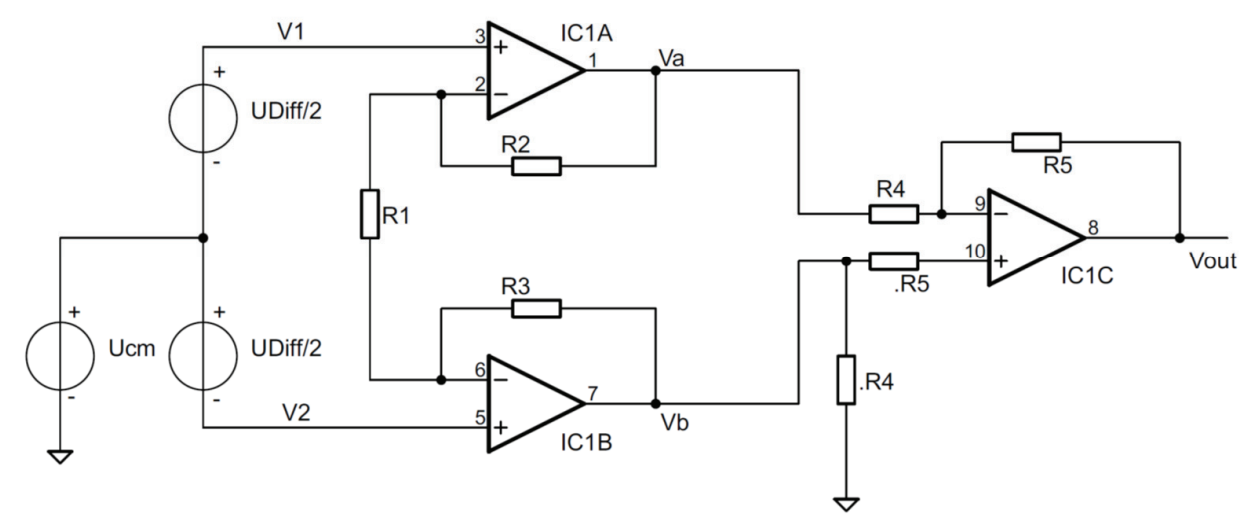

Figure 8. Basic differential amplifier circuit

$\mathrm{U}_{\mathrm{cm}}$ is the common mode voltage originating from the power grid frequency, $50 \mathrm{~Hz}$ in Europe, or may be, for example, a spur from electroluminescent lamps. Udiff is the sensor's signal, here visualized as two sources with a common mode source in the middle. Vout is defined by $\mathrm{Va}-\mathrm{Vb}$ while the overall gain is determine by $\mathrm{r} 2 / \mathrm{r} 1$ and $\mathrm{r} 3 / \mathrm{r} 1$ and the final stage $\mathrm{r} 5 / \mathrm{r} 4$ combination. See Appendix B for a detailed calculation of this amplifier.

The amplifier used for reading the $\mathrm{CO}_{2}$ sensors in Chapter 4 is a modified and enhanced version of this basic approach. The final stage, here consisting of resistors r4-r5, was replaced by an analogue-to-digital converter with a multiple 6 channel differential input stage. This means that the final stage is the same for every input. A printed circuit board was developed in order to accommodate four $\mathrm{CO}_{2}$ sensors that could be measured simultaneously.

Although errors in the differential stage may be present in the final stage, they are the same for every channel and will be ruled out in the ensuing calculations, further improving the detection limit. The reference voltage used by the analogue-to-digital converter for conversion reference is used as a power feed for the potentiometric sensors. After division of this voltage, $400 \mathrm{mV}$ was used as a reference voltage for the input to the amplifiers, adding to the stability of the interface, as when a variation of the reference voltage occurs, this variation is also fed to the input of the converter, resulting in no change. 
After design and verification of the circuit, measurements were able to be started by a zero experiment to test the electronics. The signal conditioning system was placed in the measurement chamber and verified for cross-sensitivity to temperature and humidity changes. After this step, initial measurements were able to be performed as described in Chapter 4.

Although the technique described above can also be used in the detection of hydrogen using palladium nanowires, the impedance of the wires and the delta change of the wires as a result of hydrogen diffusion were detectable with a dual source meter from the Agilent 2400 series. The disadvantage is that the source meter must be placed outside the chamber, and wire connections to the substrate may contribute noise to the signal. The relative low resistance of $8 \mathrm{k} 3 \Omega$ proved to be no obstacle to measurements, although measurements were also taken with an instrumentation amplifier in the same manner as described for measurements with the $\mathrm{CO}_{2}$ detectors. Once the temperature dependence of the wires was charted, initial compensation was carried out with a separate temperature sensor introducing a difference in response time $(\tau)$, and this had serious implications for the limit of detection during temperature fluctuations. The approach chosen to minimize this effect and the results are presented in Chapter 3.

Chapter 5 follows the same strategy as presented in the previous chapters but has its focus on capacitive based sensors. Detection is done with a custom build, high resolution capacitance interface placed in the SMS. This was done after analysis of an external capacitance measurement. 


\subsection{Conclusions}

Sensors are designed to consume less power and to decrease their size, making them suitable for application in low-power wireless sensor networks (WSN) systems. The challenge is not to compromise on the sensor's sensitivity and selectivity towards the target. This work studies various options to detect the limiting factors and to improve the limits of detection. The developments in smart sensors benefit greatly from the breakthroughs that have been made in MCU improvements over the last decades. Apart from the handling transceiver functionality by the MCU other task can be performed as well, such as typical DSP functions. The DSP functionality that can be handled by the sensors MCU gets more intricate. As new MCUs are being developed, more complex DPS tasks can be fulfilled, handling more instructions and more data at with decreasing power consumption. The power demand of P2P transfer over air has stayed more or less constant over the last decades placing a focus on utilising, from the viewpoint of power efficiency, the possible DSP functions running in the smart nodes MCU. After this, the method that was used in finding the LoD bottleneck is presented in this chapter. 


\section{Chapter 2}

\section{Defining the Signal Measurement System}

Defining, designing and verifying a proper signal measurement system is a crucial step in research. In general, sensor research focusses on the sensor itself and how it behaves rather than on the exact properties of the signal processing and the signal measurement system. Journal articles often provide clues about the experimental setup in the form of a block schematic. The equipment used can be a second clue, but how it interconnects or how far away the measurement instrument is from the testing location, and whether or not this could pose a problem during measurements, can remain unanswered questions. The reason for this short description of the experimental setup is that the single focus of this study is to measure the properties of the experimental sensor in question.

The need for a well-defined signal measurement system is that sensors often suffer from cross-sensitivity. This sensitivity to variables other than the detection agent is often problematic, but can sometimes be countered when the cross-sensitivity properties are properly mapped. The countering of cross-sensitivity is not always necessary. One reason for this is that the experimental stage of the sensor does not require compensation, while another reason is that cross-sensitivity may be present but negligible relative to the signal to noise ratio, although measurements are needed to verify this. The environmental variables in the signal measurement system must be measured, and in addition to these measurements, the controls for the level of the detection agent in the chamber and the humidity and temperature are also crucial values. Although there are climate chambers of many shapes and sizes, setting the concentration of the detection agent can be problematic. Integration of the controls and readout of the sensors can also be challenging due to the commercial closed design of these products. In general, an ideal signal measurement system has the following properties:

- All parameters can be programmed using a measurement script

- Temperatures can be set in the range needed with the accuracy needed 
- The response time is as short as possible and has a 'drop dead' response

- The temperature sensor is not affected by the sensing agent

- Humidity can be set in the range needed with the accuracy needed

- The response time of the humidity sensor is as short as possible

- The humidity is not affected by the sensing agent

- The concentration of the agent can be programmed using a script

- The concentration of the agent can be verified using a reference sensor.

Broadly speaking, there are roughly three signal measurement system approaches. The first is to have none at all, and this can be valid. This approach relies strongly on the stability of the lab temperature and the ambient humidity. When more control is desired, two general solutions remain. Both of these are based on a signal measurement system with gas input and exhausts, but they differ significantly in their properties.

When analysing sensors, only two types of these signal measurement systems are generally used. One is a flow controlled solution (Doan et al., 2012) and the other is a volume controlled environment (van der Bent, Puik, Tong, \& van Rijn, 2015). A common factor in flow controlled environments is the use of mass flow controllers (MFC) as a means of controlling gasses that mix at a certain point in the measurement setup. The test sample is kept in the gas flow throughout the measurement. A volume controlled variant uses a mixing chamber where gasses are introduced with a controlled inlet amount, although the exact concentration is measured by a calibrated reference sensor inside the signal measurement system. Among the differences between the volume and flow approaches are their treatment of the gasses. Table 1 shows the relevant properties of both systems with scores presented based on the ideal signal measurement system properties listed above. 
Table 1 . The relevant properties of dynamic and static mixing chambers

\begin{tabular}{|c|c|c|c|c|}
\hline \multirow[t]{2}{*}{ properties } & \multirow{2}{*}{ Flow controlled system } & & Volume controlled system & \multirow[b]{2}{*}{ score } \\
\hline & & \multicolumn{2}{|c|}{ score } & \\
\hline Gas mixing & Automatic at entry of chamber & ++ & $\begin{array}{l}\text { Gasses need to be in constant } \\
\text { movement to ensure } \\
\text { homogenous mixture. A small } \\
\text { fan is needed inside the } \\
\text { chamber }\end{array}$ & + \\
\hline $\begin{array}{l}\text { Temperature } \\
\text { control }\end{array}$ & $\begin{array}{l}\text { Hard to control; temperature } \\
\text { lowers due to evaporation of } \\
\text { gasses }\end{array}$ & -- & $\begin{array}{l}\text { Temperature control can be } \\
\text { done with a heat exchanger } \\
\text { and can be set within } 0.1^{\circ} \mathrm{C} 1 \text { ) }\end{array}$ & ++ \\
\hline Humidity control & $\begin{array}{l}\text { Controllable; humidity can be } \\
\text { led trough a bubbler system; } \\
\text { low settling time }\end{array}$ & + & $\begin{array}{l}\text { Controllable; Humidity can be } \\
\text { set using a bubbler }\end{array}$ & + \\
\hline Computer interface & $\begin{array}{l}\text { Most MFC have a computer } \\
\text { interface and a control } \\
\text { program; interfacing of these } \\
\text { programs requires dot net or C } \\
\text { programming }\end{array}$ & - & $\begin{array}{l}\text { Computer interface can be set } \\
\text { to industry standard to ensure } \\
\text { perfect adaption by the } \\
\text { controller program running on } \\
\text { a standard system }\end{array}$ & ++ \\
\hline Accuracy & $\begin{array}{l}\text { Excellent although the } \\
\text { controllers need calibration at } \\
\text { the start of an experiment }\end{array}$ & + & $\begin{array}{l}\text { Accuracy depends on detectors } \\
\text { in the chamber or depending } \\
\text { on the volume of the inlet }\end{array}$ & $+/-$ \\
\hline $\begin{array}{l}\text { Volume of gas } \\
\text { needed }\end{array}$ & $\begin{array}{l}\text { Relative large amount of gas } \\
\text { needed for the MFC to get in } \\
\text { range }\end{array}$ & - & $\begin{array}{l}\text { Relative small amounts of gas } \\
\text { is needed to fill the chamber } \\
\text { depending on the size of the } \\
\text { chamber }\end{array}$ & + \\
\hline Exhaust of gasses & $\begin{array}{l}\text { Gasses need to be led trough } \\
\text { filtering before entering } \\
\text { environment }\end{array}$ & - & $\begin{array}{l}\text { Small amounts of gasses can be } \\
\text { directly flushed to the } \\
\text { environment }\end{array}$ & + \\
\hline $\begin{array}{l}\text { Insertion of gas to } \\
\text { be detected }\end{array}$ & $\begin{array}{l}\text { Can be done with a MFC but is } \\
\text { difficult at low concentrations }\end{array}$ & $+/-$ & $\begin{array}{l}\text { Can be done with a pump or } \\
\text { valve or other method }\end{array}$ & + \\
\hline Overall cost & $\begin{array}{l}\text { MFC are in a high price segment } \\
\text { and require maintenance; price } \\
\text { of gasses depends on type of } \\
\text { supply }\end{array}$ & - & $\begin{array}{l}\text { Low price segment valves can } \\
\text { be used with pumps }\end{array}$ & + \\
\hline $\begin{array}{l}\text { Mixture } \\
\text { degradation in } \\
\text { chamber }\end{array}$ & $\begin{array}{l}\text { Due to the constant flow of } \\
\text { gasses there is no degradation } \\
\text { of the mixture in the chamber }\end{array}$ & ++ & $\begin{array}{l}\text { Mixture in the chamber can } \\
\text { degradate in time because of } \\
\text { interaction with the objects } \\
\text { present. This means that } \\
\text { calibrated sensors in the } \\
\text { chamber are needed }\end{array}$ & -- \\
\hline
\end{tabular}


Because sensors are the subject of this study, we are interested in determining the behaviour of a sensor when a certain amount of an agent is introduced or when temperature or humidity values change. This agent or measuring gas is often referred to as a percentage of the total, parts per million ( $\mathrm{ppm}$ ) or even parts per billion (ppb). An MFC system depends heavily on the accuracy of the controllers, especially when a low concentration in the ppb range is needed. Another major problem with the use of MFCs is the temperature of the mixture. Because mixtures tend to cool down while running through the MFC, depending on the flow rate, the actual temperature of the signal chamber is hard to control, especially when a high flow is needed. Controlling the temperature in a signal measurement system that uses an MFC also has the disadvantage that the relative humidity changes after the mixing point.

When the working hypothesis requires that the temperature and humidity in the signal measurement system are of vital importance, a volume controlled system is chosen, as is the case in chapters 3,4 and 5. Thus we now examine the properties of a volume controlled system.

\subsection{Properties of the Volume Controlled Signal Measurement System}

\section{Scripting}

The usability of a signal measurement system is defined in terms of accuracy and range. Values can be set using a lever, for example. The setting of a desired value and the reference sensor implies that there is closed-loop system, as shown in figure 1.

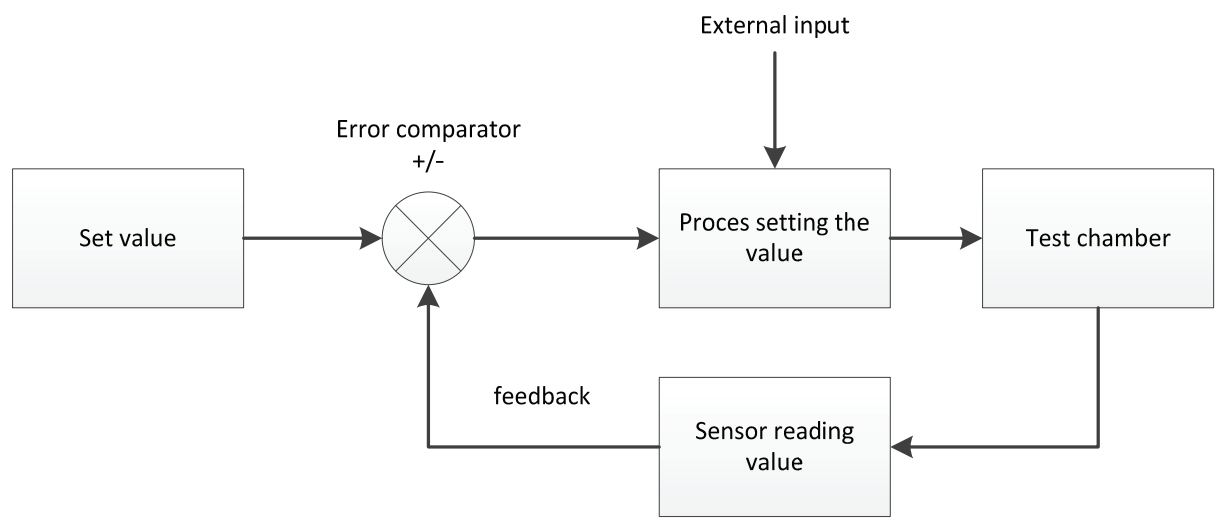

Figure 1. General closed loop control system. 
When the error comparator is digital, the process control can be performed by computer. This enables the signal measurement system to execute the script, and parameters are programmed over a certain period of time.

The format of the script file that sets the wanted values is a comma-separated text file with a txt extension. This file can be generated with $\mathrm{C}$ code, for example, but can also be composed or appended with any available text editor. The file typically has 1500 values that are executed at 1 second intervals, although the length of the script and interval time value can be modified.

The format is as follows:

[Desired relative humidity in $\% \mathrm{Rh}$, desired temperature in ${ }^{\circ} \mathrm{C}$, desired gas concentration in ppb, special function $3,6,8,7[\backslash n]]$

An example would be:

\section{$30,25,500,8[$ enter]}

$30,25,500,8[$ enter]

$30,25,500,8[$ enter]

Where 30 is a desired relative humidity of $30 \%, 25$ is a desired temperature of $25^{\circ} \mathrm{C}, 500$ is a desired gas concentration of $0.5 \mathrm{ppm}$ and 8 means normal control (see table below). The same setting repeated three times means that the settings are unchanged for 3 seconds at a script execution interval of 1 sec.

There are several functions included in the file format for testing purposes. These are:

\begin{tabular}{|l|l|l|}
\hline Function number & description & impact \\
\hline 3 & disable air inlet & $\begin{array}{l}\text { Dry and humid air inlet valves are } \\
\text { disabled. }\end{array}$ \\
\hline 6 & flush dry & Flush with only dry air \\
\hline 7 & flush all & $\begin{array}{l}\text { All inlet valves are opened in } \\
\text { order to flush the chamber }\end{array}$ \\
\hline 8 & normal operation & Test script values are executed \\
\hline
\end{tabular}

*Note that several numbers are missing. These values were chosen for script file legacy reasons in order to accept old script files from previous experiments. 
Software is needed in order to control the signal measurement system. A fully written program cannot be acquired since the application is too specific. Although software can be written from scratch at a low level such as standard C, a high-level graphical programming language facilitates connecting all parts needed for the measurement setup. These high-level graphical languages such as LabVIEW and Agilent Vee include libraries to easily interface with the GPIB or IEEE-488 industry standards used by DMM and analysers, as well as with serial communication ports. Values can be read from files and output values can be written to several formats, including Excel, for easy analysis. This functionality still needs to programmed and connected in a logical manner with the Dot Net programming environment. The measurements in chapters 3, 4 and 5 were taken with the same signal measurement setup, although every new measurement series required modifications to the Signal Measurement System. Figure 2 shows the general setup of the signal measurement system.

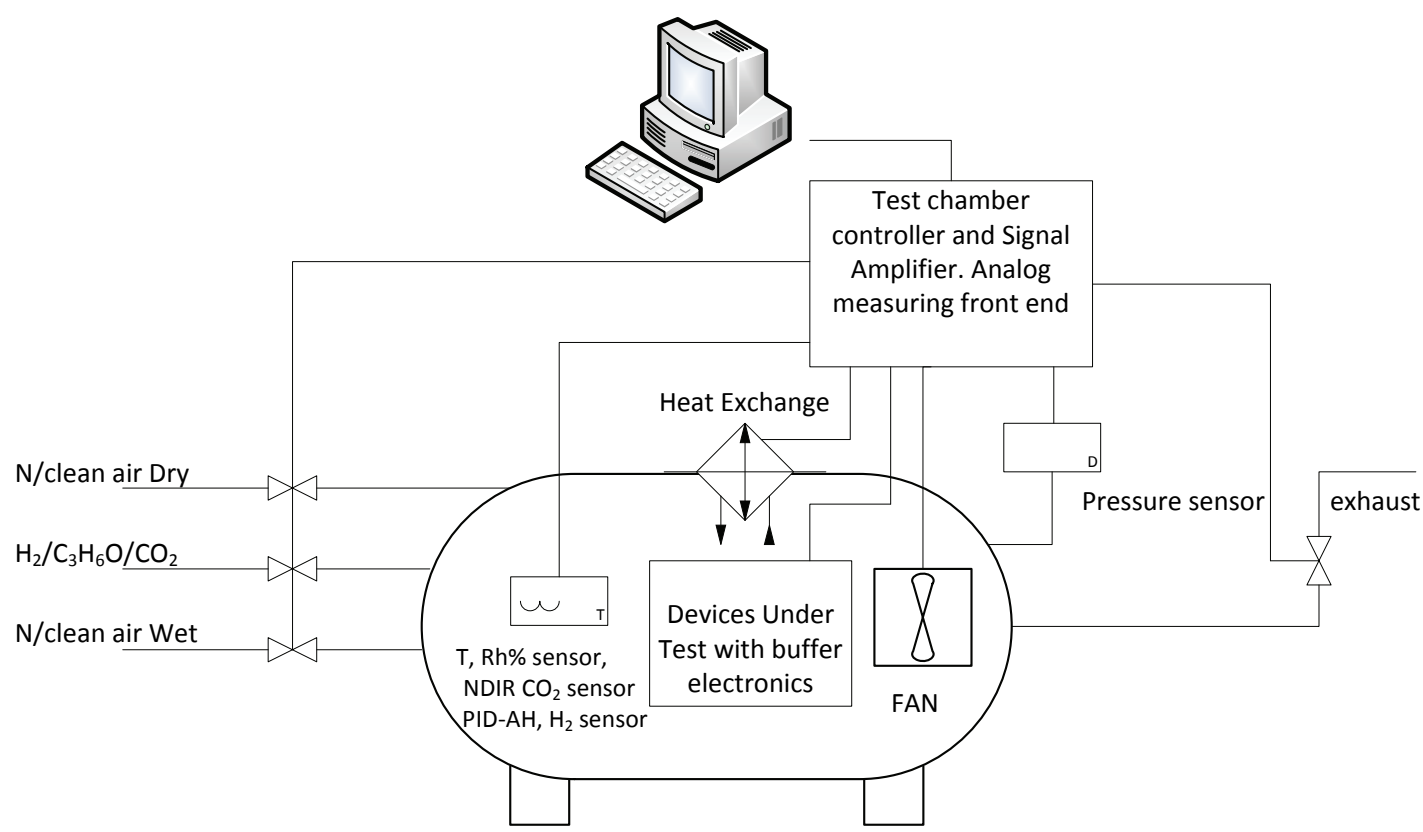

Figure 2. Complete setup of the signal measurement system

On the left side of figure 2 are the inlets. Here dry and humid air or $\mathrm{N}_{2}$ are controlled using high-speed valves which operate under the control of the PC. The exhaust of the system is also a high-speed valve. This exit valve opens when the pressure in the chamber is above ambient and closes if ambient pressure is reached. Because this is a 
one-way output, the humidity in the chamber is not influenced by the humidity outside the chamber. Temperature is controlled with a heat exchanger and a small fan inside the chamber that ensures a homogeneous mixture inside the chamber of both temperature/humidity and the gas being sensed. The frontends of the signal amplifiers are also located inside the chamber, if needed. Temperature, humidity and gas concentrations are measured with factory calibrated sensors.

\subsection{Temperature dependence of sensors}

The most common cross-sensitivity is to changing temperature. When no temperature controlled environment is available, studies usually only state that the experiments were conducted at lab temperature and standard pressure. This is commonly referred to as standard ambient temperature and pressure (SATP), with values of $298.15 \mathrm{~K}$ or $25^{\circ} \mathrm{C}$ at $100 \mathrm{kPa}$. Using a fixed operating temperature can be an excellent measurement approach, although most sensors will respond to a change in temperature. The sensors analysed in this thesis all suffer from temperature cross-sensitivity.

- Palladium, for example, is a noble metal that shrinks and expands with temperature, changing the resistance, while a change in resistance also takes place when the metal absorbs hydrogen.

- A capacitor's value will change with temperature by at least $30 \mathrm{ppm} /{ }^{\circ} \mathrm{C}$ when a C0G material is used, as dielectricum composed of rare earth metals such as neodymium and samarium (Evgeny, 2013). Any other capacitor will perform worse.

- The output voltage of potentiometric sensors, discussed in chapter 4, is determined by the Nernst equation, which factors in the temperature.

For example, every semiconductor P-N can be used as a temperature sensor and needs temperature stabilisation or compensation in order have a stable current flow over a temperature range. The principle of a P-N diode bridge inherently includes the temperature.

$I_{d}=I_{s} \cdot\left(e^{\left(\frac{V_{d}}{\eta V_{t}}\right)}-1\right)$

(Equation 1) 
Where the current through the diode is dependent on $\mathrm{V}_{\mathrm{T}}$ where $\mathrm{V}_{\mathrm{T}}=\mathrm{kT} / \mathrm{q}$ where $\mathrm{k}$ is Boltzmann's constant and $\mathrm{q}$ is the charge on an electron with $\mathrm{T}$ representing the temperature in degrees Kelvin (Horowitz \& Hill, 2015). This means that current ID is dependent on the temperature. The disadvantage of using a single diode for temperature sensing is non-linearity. This problem, however, is solved by many semiconductor manufacturers. The selection criteria for the temperature sensor are the required accuracy and calibration combined with the readout principle. The advantages of this cross-sensitivity are that it is well defined and equation 1 can be used to anticipate changes in current due to temperature variations. The relationship between temperature variations and sensor output can be more problematic and must therefore be properly measured and defined.

\section{The Measurement System}

\subsection{Temperature Control Loop}

The control loop of the temperature control system in the chamber is shown in Figure 3. The temperature sensor is a digital SHT-15 sensor from Sensirion semiconductors. This pre-calibrated sensor can measure temperature with a resolution of $0.01^{\circ} \mathrm{C}$, although the repeatability is defined with a maximum of $\pm 0.1^{\circ} \mathrm{C}$. This sensor was selected because it combined a steady long term drift of $<0.04^{\circ} \mathrm{C} / \mathrm{yr}$ and a response of $<30 \sec (63 \%)$, combined with a 14 bit digital readout and factory calibration.

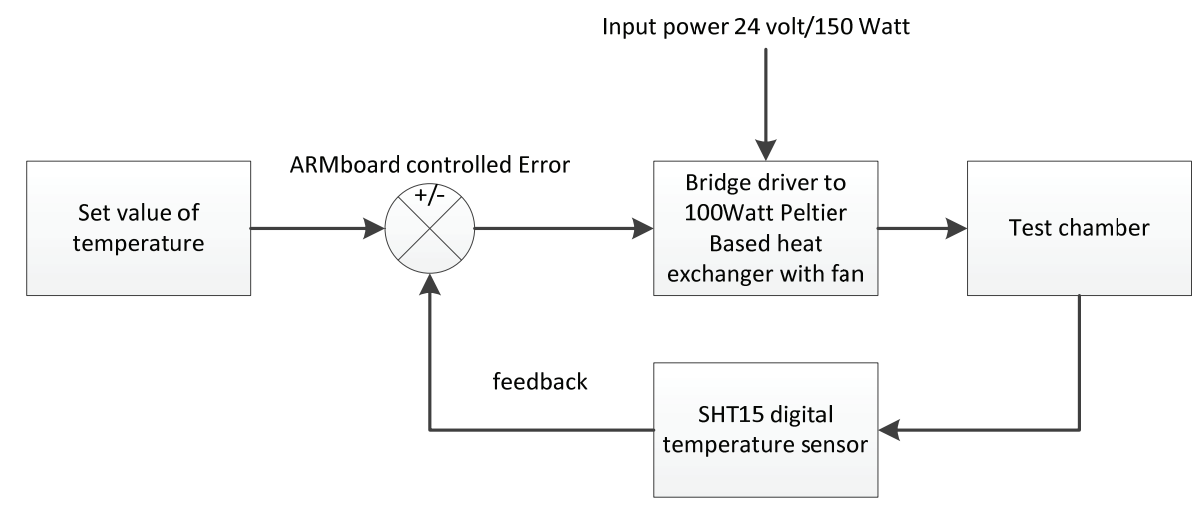

Figure 3. Simplified flowchart of the temperature control loop

The temperature in the chamber is controlled using a $100 \mathrm{~W}$ Peltier element that is controlled by an MCU, ARMboard controller and a power bridge. The bridge is driven by 
a pulse width modulated signal calculated by the ARMboard temperature controller running a proportional-integral-derivative (PID) controller. A custom controller MCU board task was used instead of a control PC output due to the differential drive of the Peltier element running at $20 \mathrm{kHz}$. This frequency cannot be achieved by the controller PC and PID values will rely on the execution speed of the control PC. In order to control the temperature, a serial RS232 interface was used to set the values of the temperature controller. The control PC (see Figure 2) sets the value of the temperature by script intervention. Figure 4 shows the chamber response to an input script. The programmed temperature response is pyramidal and has a span of $20^{\circ} \mathrm{C}$.

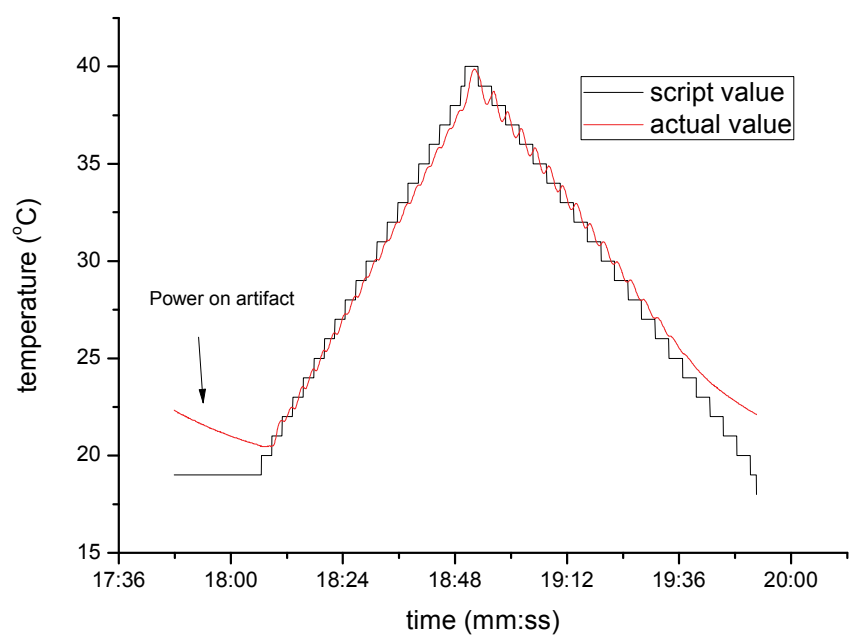

Figure 4. Temperature response of the signal measurement system to a script input

The total duration of the script depends on its length and the execution speed set by the control PC. In this case, a total of more than two hours is needed to complete the whole sequence. At every cycle all variables are read and sent to a software application that builds the output file structure. Apart from setting the temperature value, the single script file also includes the humidity values and the gas concentration values in the chamber. 


\subsection{Humidity control loop}

The measurement of the humidity as a key element in the closed loop control (see figure 5 ) is the reference sensor and the accuracy. There are a number of variables that can conflict. The settling time of the total signal measurement system will always be smaller than the settling time of the sensor. Other factors are resolution, factory calibration and resistance to volatile compounds. In the measurements presented in chapters 3 and 4, the SHT-15 integrated humidity sensor was used.

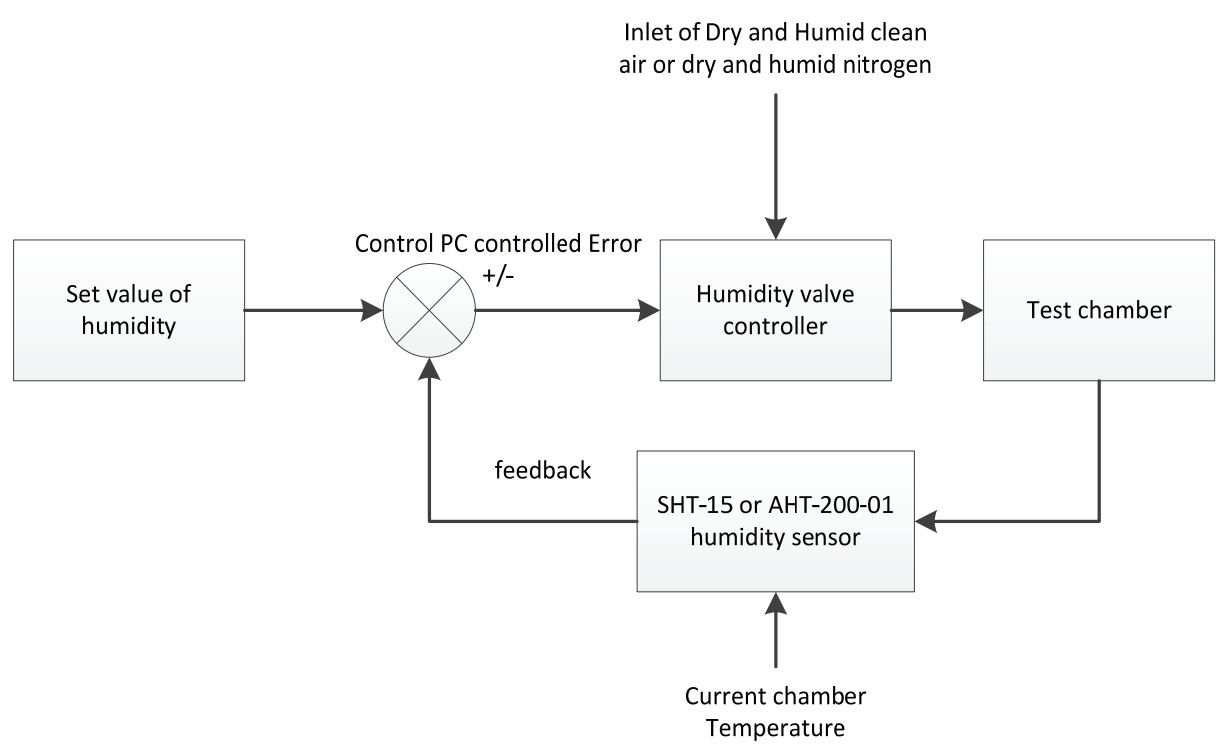

Figure 5. Simplified flowchart of the humidity control loop

Since volatile chemical vapours affect the readout of the humidity sensor and may damage the sensor in the short or long term, it is useful to review the underlying principles. In literature, referenced humidity sensors often aim for high resolution combined with factory calibration. The logic of this approach is evident, but the downside is that the majority of the commercially available humidity sensors do not respond well to volatile chemicals such as ketones and hydroxyls. In general, there are three commercially available sensor principles (Farahani, Wagiran, \& Hamidon, 2014; Sensors_online, 2001) to measure humidity. The SHT-15 compensates internally for temperature changes, while the AHT-200-01 needs external temperature compensation. The external temperature value is supplied with the aid of the SHT-15 used in chapter 5. 


\subsubsection{Capacitive Sensors}

The most common solution to sensing Relative Humidity (Rh\%) is a capacitive sensor. This principle of sensing involves a small film of polymer between electrodes as a capacitor. Interaction with water vapour changes the value of the capacitor and provides a small change of 0.2 to $0.5 \mathrm{pF}$ for a $1 \% \mathrm{Rh}$ change of the bare IDE value of the capacitor, that is between $100 \mathrm{pF}$ and $500 \mathrm{pF}$ at $50 \% \mathrm{Rh}$ at $25^{\circ} \mathrm{C}$. This type of sensor has a near perfect linear response but requires calibration because of the spread in bare IDE capacitance. The Sensirion SHT15 sensor was used in the previous setup and provided sufficient precision at $2 \% \mathrm{Rh}$ and $0.5^{\circ} \mathrm{C}$ accuracy over the whole range from $-20^{\circ} \mathrm{C}$ to $120^{\circ} \mathrm{C}$, with a digital readout to minimize signal conversion errors. Another positive aspect is the long lifespan ( $>10$ years) of this sensor. In Chapter 5 experiments with acetone sensitive IDE sensors are conducted in an acetone environment with $1000 \mathrm{ppm}$ concentrations. Here the SHT15 is replaced by another sensor that can withstand this harsh environment.

\subsubsection{Resistive Sensors}

Resistive humidity sensors measure the impedance change of a hygroscopic layer based on media such as conductive polymers or salt. They are far from linear to humidity changes and typically have an inverse relationship to humidity. This property requires the sensor to be calibrated before use. The life expectancy of this sensor is less than 5 years when exposed to chemicals causing uncertain deterioration of the sensor's properties. The accuracy of this sensor type is $\pm 2 \% \mathrm{Rh}$. This sensor could be used although it is uncertain how the sensor would respond to low concentrations $(<100 \mathrm{ppm})$ of ketones in the long run. 


\subsubsection{Thermal Conductivity Humidity Sensors}

The working principle of this sensor type is based on the difference between the thermal conductivity of dry air and air that contains water vapour. Such sensors consist of two

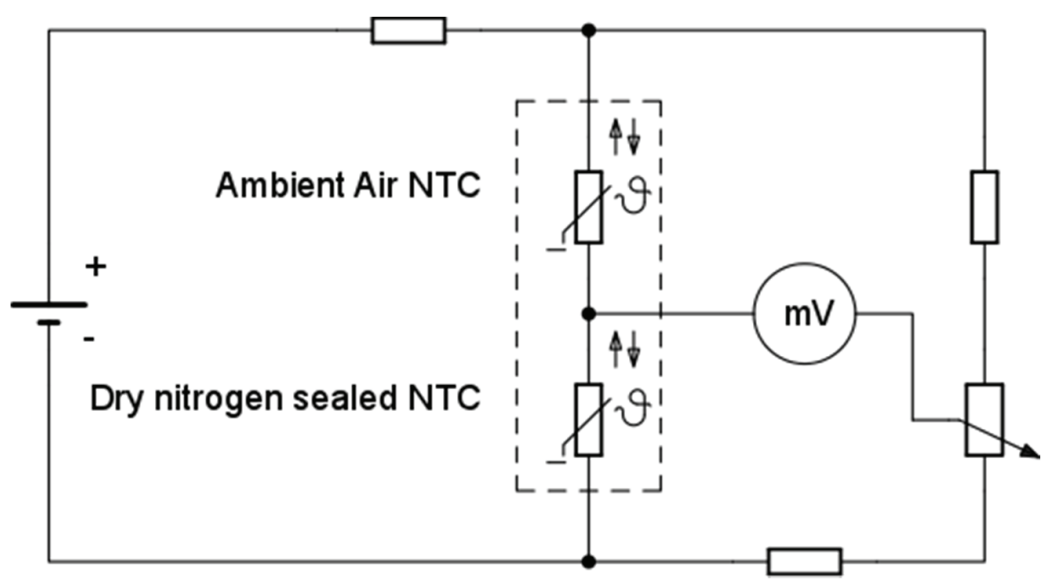

Figure 6. Working principle of Thermal Conductivity Humidity Sensors.

matched negative temperature coefficient (NTC) thermistors. One is sealed in dry nitrogen and the other is exposed to the environment in a bridge setup (see figure 6).

When a current is fed though the thermistors, resistive heating occurs, increasing the temperature to more than $200^{\circ} \mathrm{C}$. The heat that is dissipated from the sealed thermistor is greater than the thermistor that is exposed to the water vapour due to the higher thermal conductivity of the water vapour compared to the dry nitrogen. The difference in dissipated heat is a measure of the absolute humidity. This sensor type is often used in industrial environments and can withstand an acetone environment. The downside is that these sensors heat up and can therefore influence the measurement. Their place in the signal measurement system should be chosen with care. Furthermore, this sensor requires calibration and has a typical accuracy of $\pm 5 \%$ at $40^{\circ} \mathrm{C}$ and a $\pm .5 \%$ at $100^{\circ} \mathrm{C}$. Apart from the calibration and the nonlinear accuracy of this sensor type, it is the most promising for measuring in harsh environments where acetone, for example, may be used. This sensor type can also be used for measuring in a $\mathrm{CO}_{2}$ or $\mathrm{H}_{2}$ environment, although the SHT-15 is also suitable. 


\subsubsection{Other Systems To Measure Humidity}

Apart from the systems mentioned above, there are other ways of measuring humidity. Most of these techniques, such as the chilled mirror, are both experimental and costly. The reliability and accuracy are very good. The working principle of this method is the detection of the dew point on a temperature-controlled mirror. Another option is the optical hygrometer. This works on the principle of absorption of radiation at a specific wavelength. This type of hygrometer is used as a reference for calibration of other systems (Kahn, july 2012), and the dew point output is a derivative of relative humidity.

\section{5. $\mathrm{H}_{2}$ control loop}

Chapter 3 shows the improvements of the limits of detection of an $\mathrm{H}_{2}$ sensing system using palladium nanowires. In order to set the concentration of $\mathrm{H}_{2}$ in the chamber, another control loop was used. Figure 7 shows the closed loop control system of the $\mathrm{H}_{2}$ inlet.

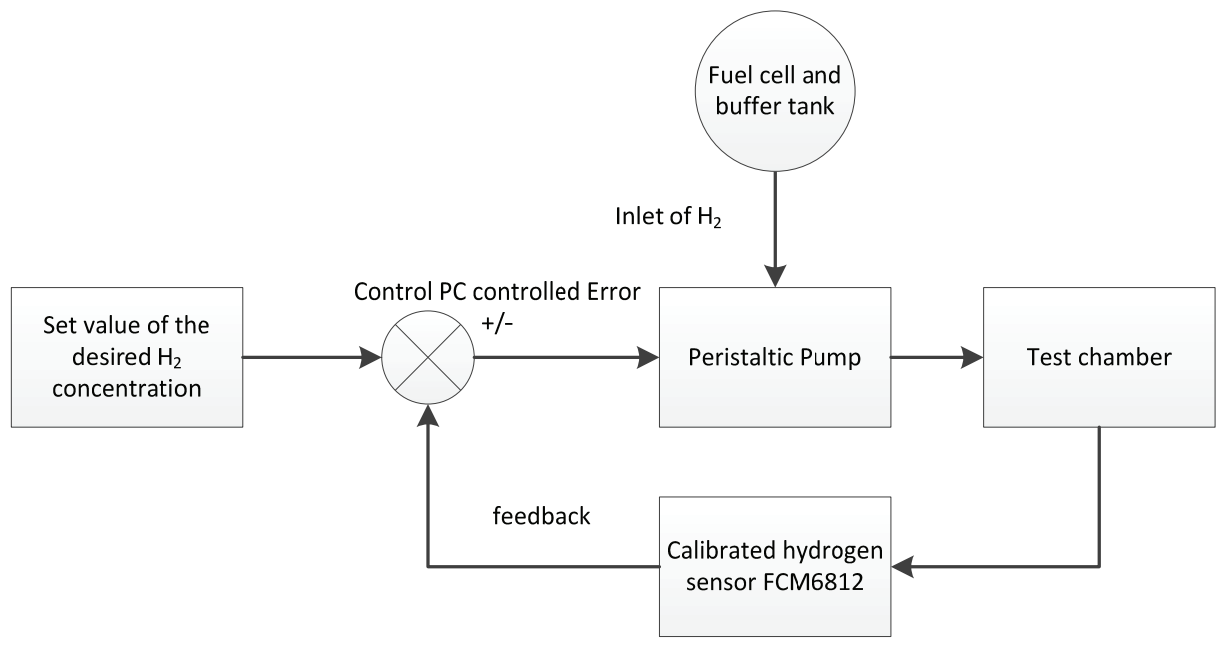

Figure 7. Simplified flowchart of the hydrogen control loop

The control PC in charge of setting all the values and running the main program compares the desired value with the current concentration and is able to change the concentration in the signal measurement system by actuating the peristaltic pump fed by a fuel cell that creates $\mathrm{H}_{2}$ and stores the gas in a buffer. Every rotation of the peristaltic pump adds to the concentration of $\mathrm{H}_{2}$ in the signal measurement system. The concentration is monitored by a calibrated Figaro FCM6812 $\mathrm{H}_{2}$ sensor from, which is able to convert the concentration to a voltage. This voltage is then measured by a 
calibrated DMM and fed back to the control loop. The script file is read by the control PC and this device in turn sets the concentration. In this way, the exact input of $\mathrm{H}_{2}$ is not measured by counting the number of rotations of the peristaltic pump, but depends on the calibrated $\mathrm{H}_{2}$ sensor inside the chamber.

\section{6. $\mathrm{O}_{2}$ control system}

In chapter 4 the measured gas is $\mathrm{CO}_{2}$, which means that the chamber must be modified to accommodate the inlet and control system. Again, the setup is a closed loop system and the $\mathrm{CO}_{2}$ was injected into the chamber by a controlled valve as shown in figure 8.

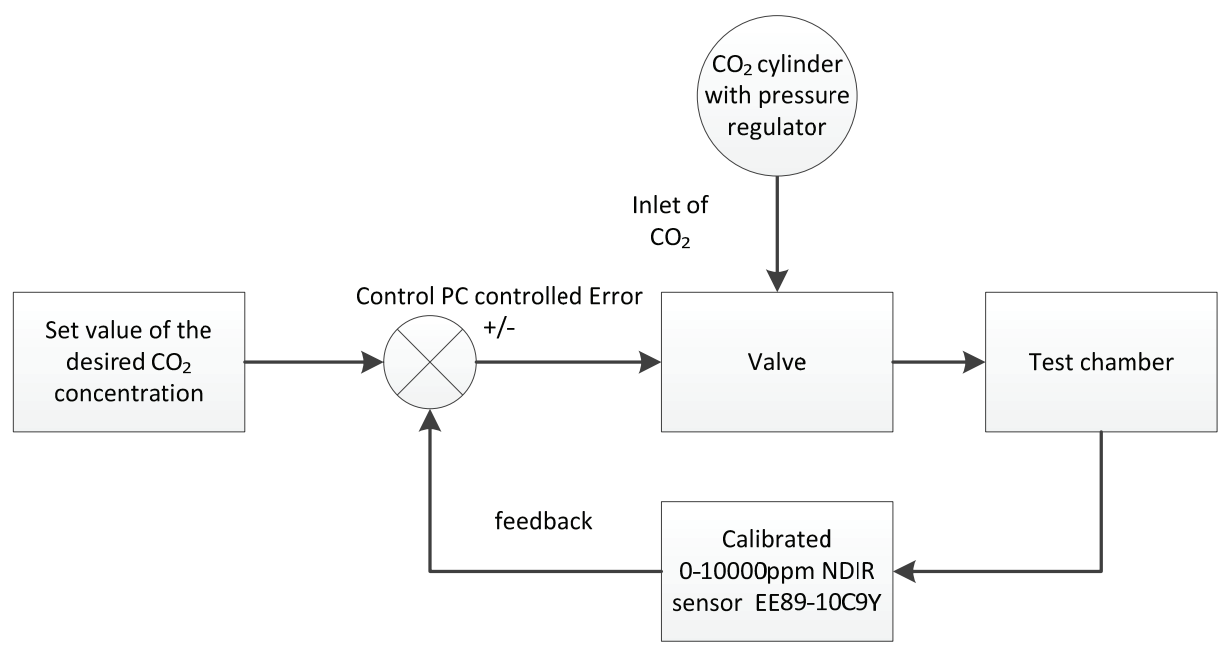

Figure 8. Simplified flowchart of the $\mathrm{CO}_{2}$ control loop

As was also the case with the $\mathrm{H}_{2}$ setup, the resolution of this closed-loop system also depends on the $\mathrm{CO}_{2}$ reference sensor. The calibrated EE89 sensor from E+E devices is a nondispersive infrared sensor (NDIR) and was chosen because of the outstanding resolution and reproducibility offered by this type of spectroscopic sensor. The sensor was placed in the measurement chamber and the feedback loop included a digital signal that was interfaced using the ARMboard with a modified $\mathrm{I}^{2} \mathrm{C}$ protocol. The feedback signal is fed into the control PC program that regulates the input of $\mathrm{CO}_{2} \cdot \mathrm{CO}_{2}$ was administered using a high-speed valve connected to a cylinder of $\mathrm{CO}_{2}$ with a pressure regulator. The amount of $\mathrm{CO}_{2}$ injected in the chamber is regulated with a controlled open/close time of the valve. 


\subsection{The Control PC Program}

As described above, the PC control program was written in a graphical programming language. Although the system was modified to accommodate all the different measurements, Figure 9 presents a general overview of the software system using the IDEF0 (Zaal, 2009) description method. All dependencies of the signal measurement system are shown, from the input script file to the output builder block of the Excel file.
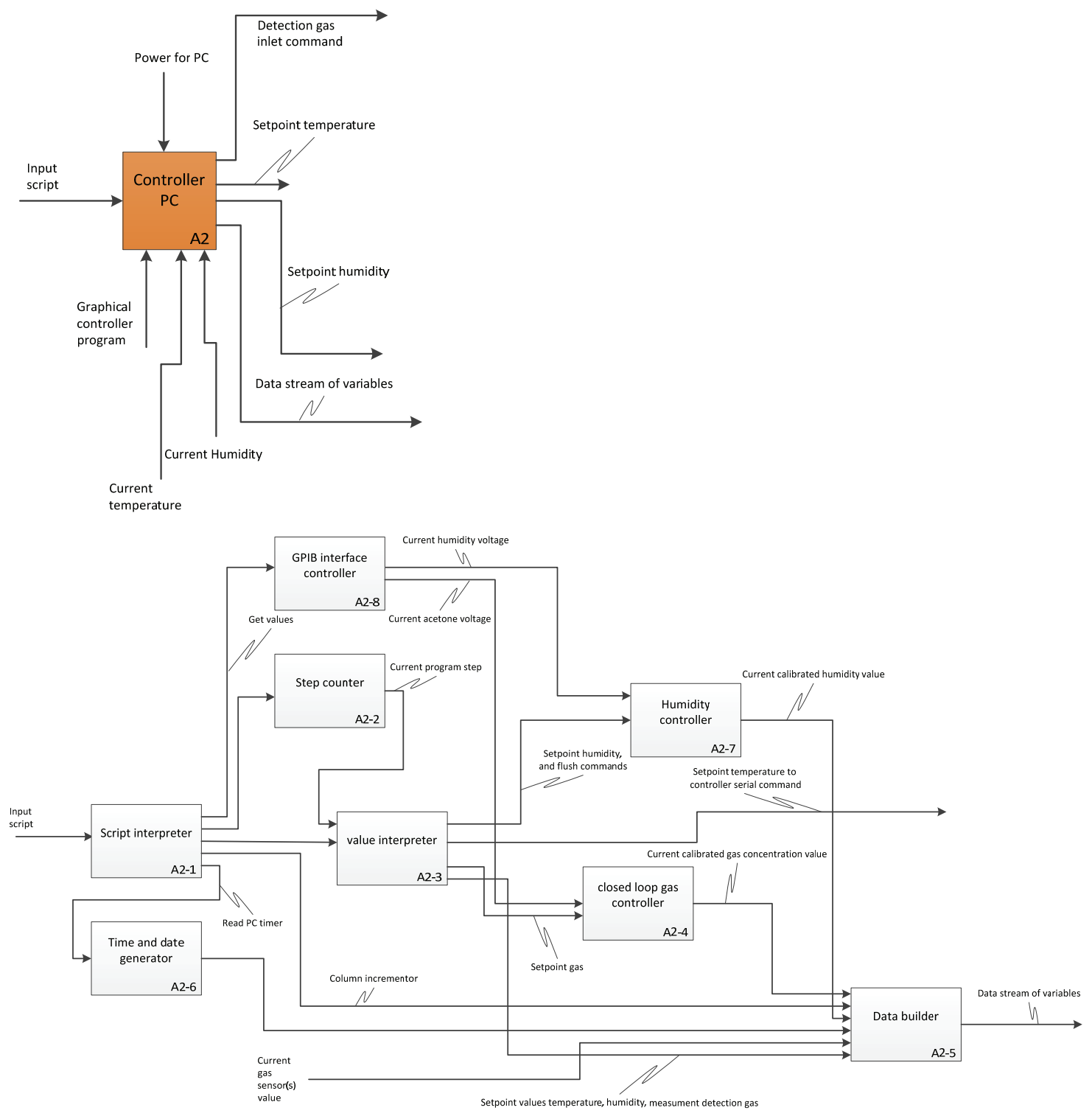

Figure 9. IDEF0 model of the control PC software

In this software the A2-1 block is the main trigger. This block reads the script file and sets the execution speed. This speed also determines the sampling frequency of the 
system variables, although data collectors in frontends are equipped with commands for faster sampling up to $100 \mathrm{kHz}$. The time mark is retrieved from the PC timer and stored in the data builder, the A2-5 block, which collects and distributes data points to their place in the Excel file. The gas and humidity controllers are regulated by this software while the temperature controller is placed outside of the signal measurement system. Also present and used when needed is block A2-8, the GPIB controller, which is connected to a DMM that measures the humidity in the system. A total of three independent loops perform their tasks in this system. All the actual values are measured with calibrated sensors and constitute the key elements in this system. Since high-speed valves are used in this system, the pressure of clean air at the input also determines stability of the set point. When a low input pressure is selected the set values are reached over a long period of time but with excellent precision, and when a high input is chosen, the precision is lower but the response time is faster. The setting that is chosen depends on the properties of the sensor being tested. If long-term stability is the research subject, for example, the flow is set low. When response times are to be tested, a high flow is selected with less accuracy in the end value.

\subsection{Conclusions}

Before any countermeasures can be taken to improve the LoD, all relevant parameters of the sensor in question must be obtained. For example, if a sensor is to be used outdoors, it must be studied with the relevant humidity and temperature ranges. This means that a signal measurement system needs to be designed, constructed and verified that is capable of recreating these conditions in a controlled manner. In order to do this, the commonly known method of mixing with MFC was analyzed. This method proved to be ineffective with respect to controlling the humidity and the temperature of the mixture. A volume controlled system was designed to accommodate all the requested characteristics. Most settings, such as temperature, are designed with control loops that can be supplied with the desired value by means of a script file. This method ensures that experiments can be repeated, while maintaining the measurement conditions, multiple times. 


\section{Chapter 3}

\section{Temperature Balanced Hydrogen Sensor System with Coupled Palladium Nanowires 1}

\section{Overview}

A temperature compensated hydrogen sensor was designed and made capable of detecting $\mathrm{H}_{2}$ within a broad range of 100-10,000 ppm while compensating instantaneously for large $\left( \pm 25^{\circ} \mathrm{C}\right)$ temperature variations. Two related operational constraints were simultaneously addressed: 1) selective and sensitive detection under large temperature changes, and 2) fast warning at low and increasing $\mathrm{H}_{2}$ levels. Accurate measurements of hydrogen concentrations were ensured by matching relevant timeconstants. This was achieved with a microchip having two temperature coupled palladium nanowires. One of the $\mathrm{H}_{2}$ sensitive $\mathrm{Pd}$ nanowires was directly exposed to hydrogen, while the other nanowire was used as a temperature sensor and a reference. A drop forging technique was used to passivate the second Pd wire against $\mathrm{H}_{2}$ sensing. Temperature effects were substantially reduced with a digital signal processing algorithm. Measurements were taken in a signal measurement system, enabling the hydrogen concentration to be controlled over both short and long periods. An early response for $\mathrm{H}_{2}$ sensing is attainable on the order of 600 milliseconds, and an accurate value for the absolute hydrogen concentration can be obtained within 15 seconds.

\subsection{Introduction}

Palladium is a good candidate material to detect hydrogen (Atashbar, Banerji, \& Singamaneni, 2005; Cheng, Li, Lisi, \& Wang, 1996; Favier, Walter, Zach, Benter, \& Penner, 2001; Kyun Tae, Jun, \& Sung Min, 2006) by measuring the electrical conductance.

\footnotetext{
${ }^{1}$ Accepted and published by the journal Sensors and Actuators A: Physical; Co-authors: Puik, Tong and van Rijn
} 
One-dimensional structures such as nanowires are also particularly compelling for sensing applications due to their suitability for large-scale high-density integration and high sensitivity to surface interactions. Because of the high ratio of 'surface area' to 'bulk volume' of nanowires, the interaction time constant is small and enables saturation of the wire on the order of less than a second.

To this point, various one-dimensional structures such as $\mathrm{ZnO}, \mathrm{GaN}$, and core-shell nanorods have been synthesized by different methods using a bottom-up fabrication technique, and then used to further build up ultrasensitive hydrogen sensors which operate at room temperature (Abdullah et al., 2013; Abdullah, Yam, Hassan, \& Bououdina, 2014; Barreca et al., 2010; Huang \& Lin, 2012). Although these bottom-up synthesized hydrogen sensing devices are reported to have excellent sensitivity to hydrogen, they may have a cross-sensitivity towards other gases ( $\mathrm{NH}_{3}$, for example), humidity etc. (cf. palladium, the only material having no cross-sensitivity to other agents, even water vapour). Current methods of manufacturing $\mathrm{ZnO}$, GaN, and core-shell nanorods also do not provide controlled growth of high-density ordered arrays, and transfer and assembly of devices on separate substrates are typically required, meaning that reproducibly forming high-quality devices has been quite challenging (Atashbar et al., 2005; Cheng et al., 1996; Favier et al., 2001; Im et al., 2006).

Top-down approaches, on the other hand, are more straightforward for fabricating onedimensional structures. The techniques used here are mainly thin film deposition, patterned etching, and/or ion-beam milling of planar substrates. Patterning is typically done using UV- and DUV-photolithography and is commonly combined with advanced nanolithography techniques such as electron beam lithography (EBL), focused ion beam (FIB) or nano imprint lithography (NIL). Feature sizes down to a few nanometres can be achieved by these techniques (Offermans et al., 2009).

Because the temperature also influences the electrical resistance, a thermal compensation technique is needed. Adequately correcting for temperature fluctuations is a challenge due to the small interaction time constant of the nanowires. Note that the time constant for the temperature compensation circuitry should ideally match the thermal time constant of the nanowire. Other research groups have also reported the 
use of palladium nanowires as a suitable sensing material (Atashbar et al., 2005; Joshi, Krishnan, Yoshimura, \& Kumar, 2009; Kyun Tae et al., 2006; Offermans et al., 2009; Wolfe, Love, Paul, Chabinyc, \& Whitesides, 2002) and some have reported considerable responses to temperature variations. Under lab conditions with good temperature control (better than $1 / 20^{\circ} \mathrm{C}$ ) a sensitivity of $30 \mathrm{ppm}$ was obtained in a broad range of 100-200,000 ppm (Offermans et al., 2009). The effect of a temperature change of only +1 ${ }^{\circ} \mathrm{C}$ is comparable with a 500-600 ppm change in the $\mathrm{H}_{2}$ level. This means that the temperature effect of $1{ }^{\circ} \mathrm{C}$ is approximately 20 times greater than the best achieved detection limit under lab conditions. Straightforward temperature compensation can be achieved by measuring the temperature nearby or on the substrate, and also by a detailed calibration procedure. We present another strategy here, however.

We propose a temperature counterbalancing method that uses two nanowires on the same substrate for compensation of the temperature effect in the readout. With the suggested method, a detection limit of $100 \mathrm{ppm}$ is achieved even with erratic temperature fluctuations. We report a detection limit that is 400 times less than the lower explosion level of 4\% (CHEMSAFE, 2004), thus enabling selective and sensitive detection of $\mathrm{H}_{2}$ for autonomous sensor networks.

A 'drop forging' technique is introduced by coating a second Pd nanowire on the same substrate with a sufficiently thin passivation layer, resulting in a reference/temperature sensor with an adequate time response. Passivation of sensor elements is well established as a compensation procedure (Fleischer et al., 2001). Here the passivation layer should operate as a functional barrier for $\mathrm{H}_{2}$ without changing the relevant time constants.

\section{Materials and Methods}

\subsection{Fabrication of the Palladium Nanowire Chip}

A dual palladium nanowire chip was fabricated using the innovative process of deposition and etching under an angle (DEA) (Tong, Chen, Wiel van der, Carlen, \& Berg van den, 2009). DEA is a novel fabrication process with the ability to produce Pd wires with lengths up to several $\mathrm{mm}$ and widths as low as $20 \mathrm{~nm}$ using standard CMOS 
microfabrication processes. The detailed process flow to fabricate PdNWs is shown in Figure 1a. A layer of $1000 \mathrm{~nm}$ silicon dioxide $\left(\mathrm{SiO}_{2}\right)$ is grown on a 4 inch (100) silicon wafer by means of wet oxidation. Conventional microlithography is then carried out to define patterns on the wafer, followed by isotropic etching of $\mathrm{SiO} 2$ for 50 seconds in a buffered oxide etching (BHF) solution. By under-etching, this isotropic etching creates a nano-cavity with a width of about $55 \pm 5 \mathrm{~nm}$ below the photoresist layer. Layers of $40 \mathrm{~nm}$ Pd preceded by a $10 \mathrm{~nm}$ adhesive tantalum (Ta) are then deposited by an E-beam evaporator with an inclined angle of $45^{\circ}$ on the surface of the patterned wafer. The typical E-beam evaporation rate is $1 \AA \mathrm{s}-1$ for both Ta and Pd. As a result of the inclined deposition, a small part of the $\mathrm{Pd} / \mathrm{Ta}$ is deposited into the nano-cavity and hidden below the photoresist film. Subsequently, argon (Ar) ion beam etching (RIBE, Oxford i300) is carried out to pattern the deposited Pt/Ta film on the silicon wafer. The metallic parts hidden below the photoresist film are not reached by the Ar ion beam and remain as metallic parts with a width of about $60 \pm 5 \mathrm{~nm}$, thus forming the metallic Pd/Ta nanowires (figure1b). Finally, the wafer containing Pd/Ta nanowires is diced into small chips with a typical size of $7 \times 7 \mathrm{~mm}$ (figure $2 \mathrm{a}$ ). Each chip consists of a double array of 14 Pd nanowires with different lengths. 
1. Photoresist patterning

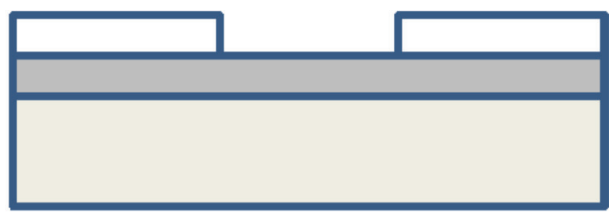

2. Isotropic etching $\mathrm{SiO}_{2}$

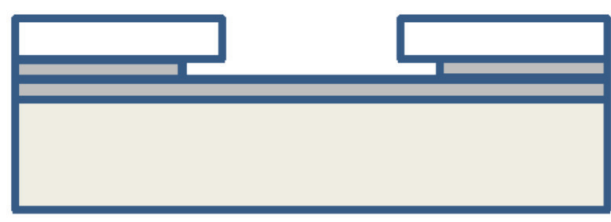

3. Evaporated $\mathrm{Pd} / \mathrm{Ta}$ under angle $\left(45^{\circ}\right)$
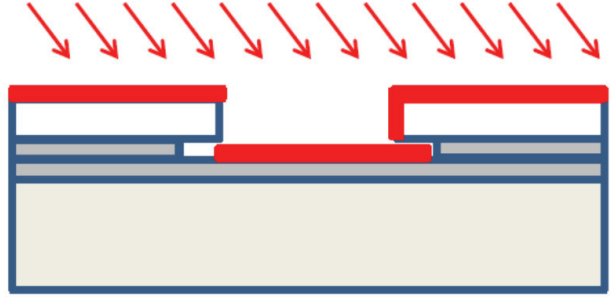

4. Ion beam etching of metal ( angle of $10^{\circ}$ )

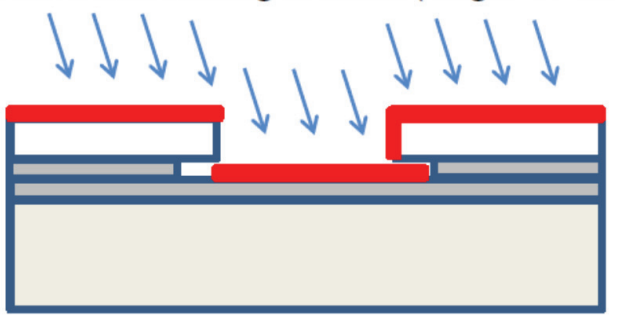

5. Metal part hidden below resist remained

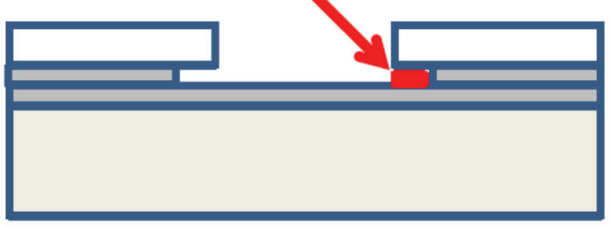

6. Removing the resist in hot acetone, Revealing wafer-scale Pd nanowires

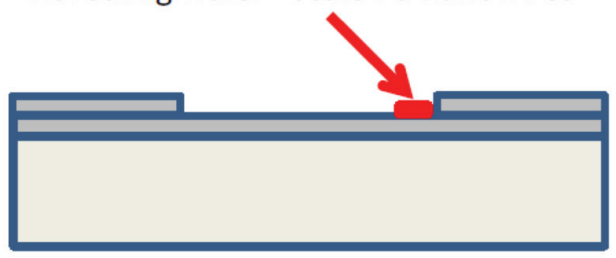

Figure 1.A. DEA fabrication process to make wafer-scale Pd nanowire using a deposition and etching under angle process. 


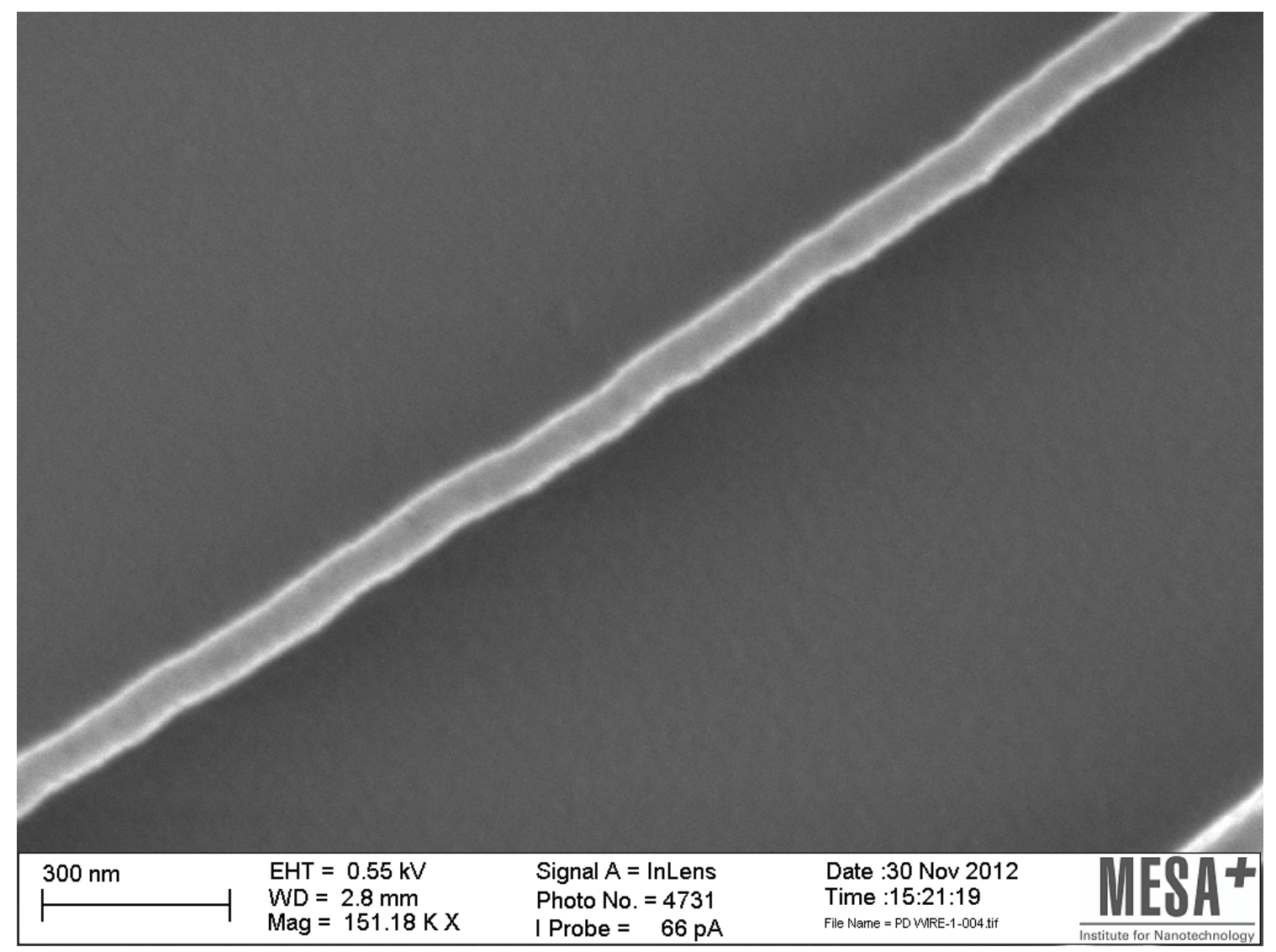

Figure 1.B. SEM image of Pd nanowire, showing a high resolution scanning electron microscopy (HR: SEM) image of the fabricated Pd nanowire. The nanowire produced has a width of about $60 \pm 5 \mathrm{~nm}$.

By adjusting several processing parameters such as the dimensions of the nano-cavity (by varying the $\mathrm{SiO} 2$ isotropic etching step) and inclining angles during metal film deposition and IBE etching, Pd nanowires with various widths can be obtained. In the current work we optimized the process parameters to obtain Pd nanowires with a width of around $60 \mathrm{~nm}$, because wider nanowires may reduce the sensors' sensitivity, while narrower ones may provide high sensitivity but also have elevated external noise levels. 


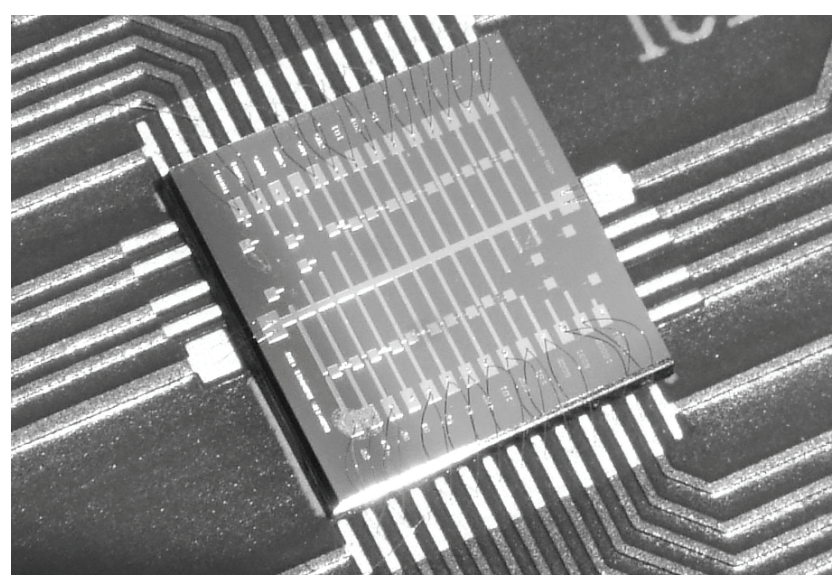

Figure 2.A FR4 PCB circuit board with nanowire chip

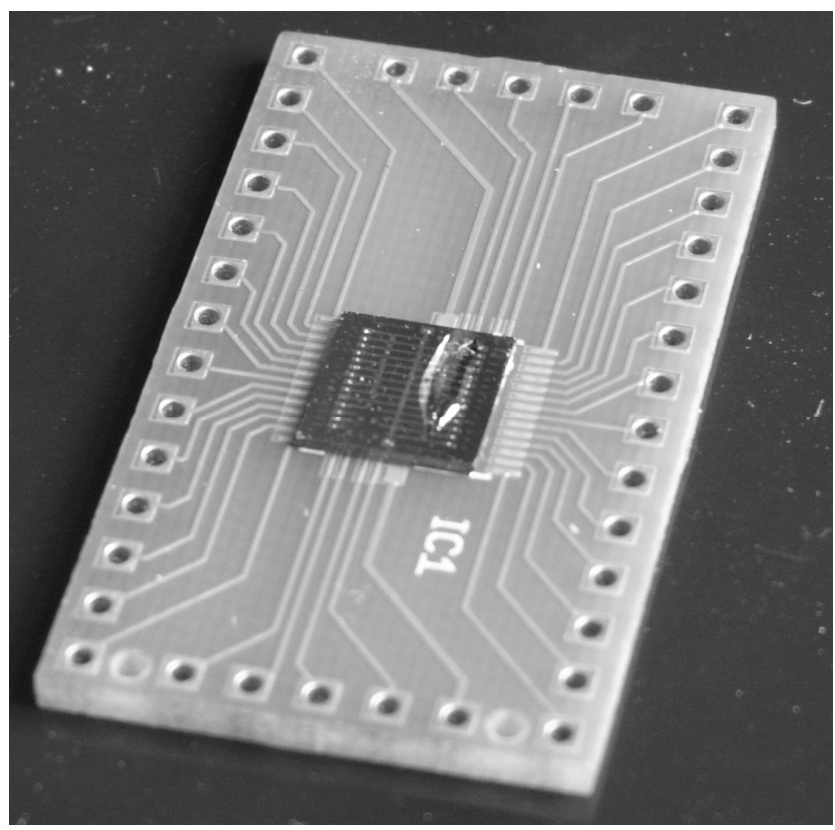

Figure 2.B Close-up of the nanowire chip after drop casting

The chips were then visually inspected with an optical microscope, after which they were affixed to a custom FR4 printed circuit board (PCB) (Fig 2.a) with a gold plated top layer. One half of the nanowires were covered with a UV dryable 2-hydroxyethyl methacrylate-based compound (Fig 2.b) using drop casting. For this purpose, a syringe with a $0.5 \mathrm{~mm}$ flat needle was used to cast the drop at approximately $3-5 \mathrm{~mm}$ from the sample. After visual inspection the sample was exposed for 2 minutes to an Acticure 4000 UV source. The thickness of the layer was tested with an alpha-step surface profiler and had a maximum of approximately $400 \mu \mathrm{m}$ above the selected wire. The 
bonding of the compound to the nanowire chip was tested using a peel off with adhesive tape and this test was repeated three times. The chip and the PCB were then cleaned and processed using a wedge bonder with $35 \mu \mathrm{m}$ gold wires. The settings of the wedge bonder were separately tested with a non-destructive test of the bonds, showing at least 3 grams of strength. After the bonding process the wires were tested for stability with a Keithley 2400 source meter at $50 \mu \mathrm{A}$ and two identical wires of $8.3 \mathrm{k} \Omega$ were selected, one in the left half and one in the right half. Finally, the chip and bonding wires were protected by an open plastic cover, providing the sample with added mechanical strength.

\subsection{Measurement setup}

The sensor tests were performed in a signal measurement system in which temperature and humidity levels up to $1 \%$ could be accurately controlled (see Figure 3). The gasses in the signal measurement system were kept in constant motion using a small fan to ensure a homogeneous gas mixture in the chamber.

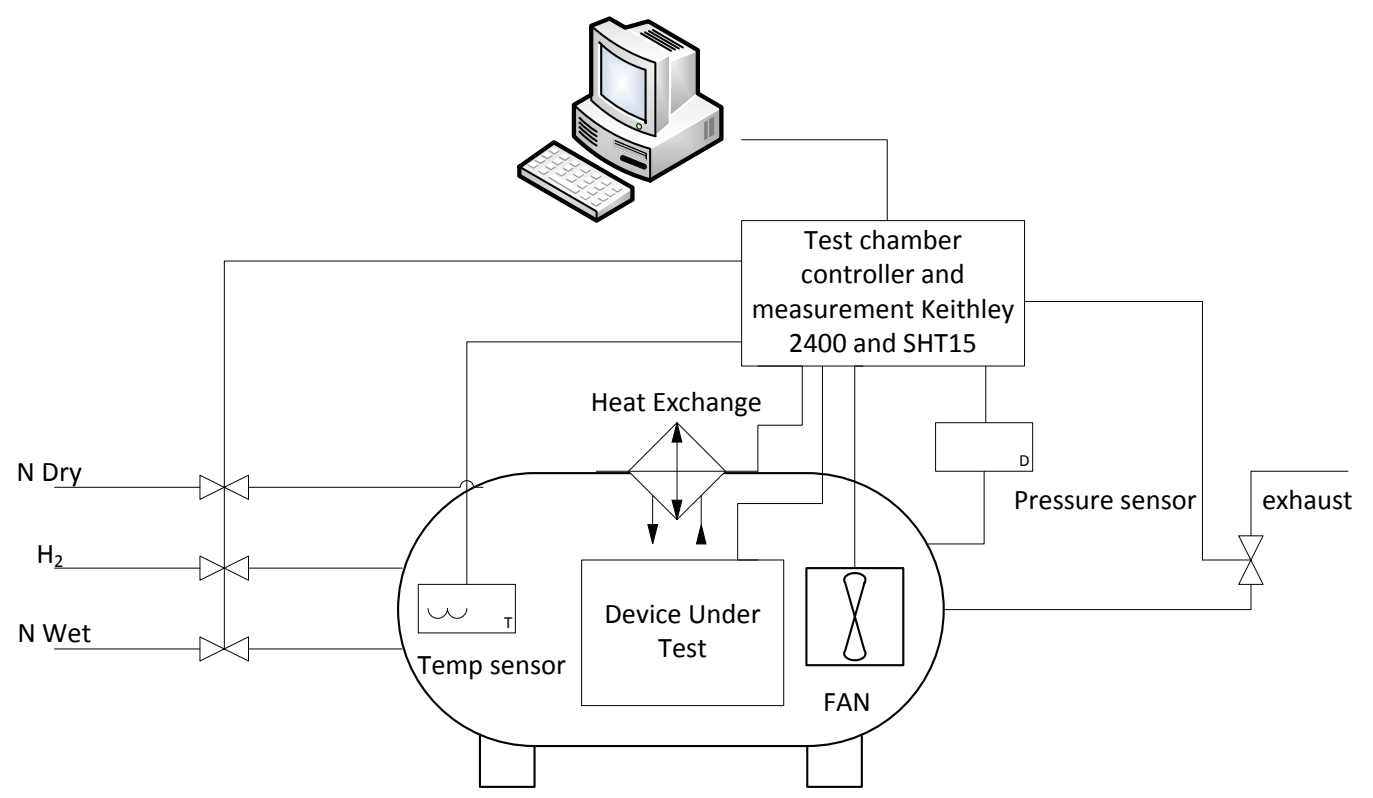

Figure 3. Schematic representation of the measurement setup

The settings of the test chamber were controlled by a Labview program and all data were recorded in a comma-separated file. The Labview software enables active settings 
for temperature, humidity and hydrogen level that can run over a long ( $>1$ week) interval. This ensures that measurements can be repeated. The measurement bias current through the nanowires was set to $50 \mu \mathrm{A}$, leading to an excitation voltage of 400 $\mathrm{mV}$ over the nanowire. Chips were tested with different concentrations of hydrogen to ensure a correct response within the 100-10,000 ppm range.

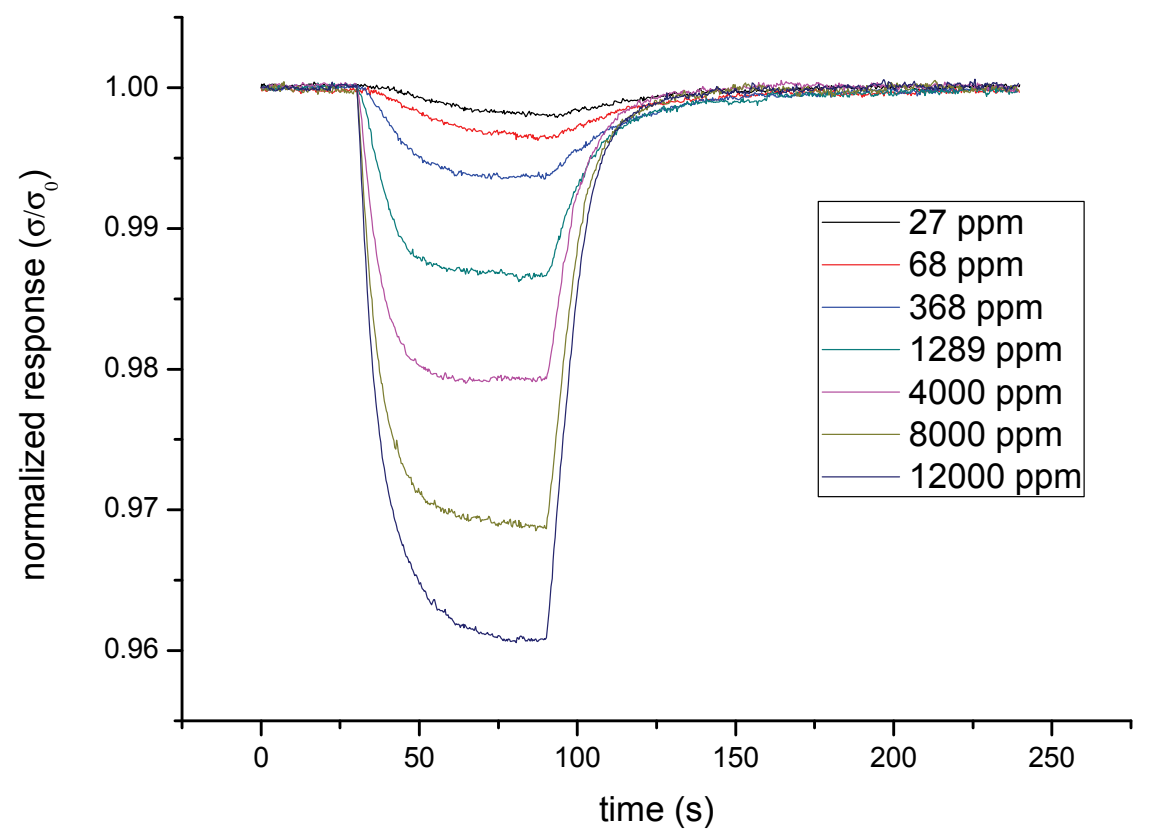

Figure 4. Change in conductance in an uncovered Pd wire at levels from $27 \mathrm{ppm}$ to 12,000 ppm hydrogen in a nitrogen environment

Figure 4 shows the response of the uncovered nanowire at a constant lab temperature where $\sigma 0$ represents the hydrogen level of $0 \mathrm{ppm}$. The relative conductance change is plotted at constant room temperature in a nitrogen environment with different hydrogen levels. With a bias current of $50 \mu \mathrm{A}$, the response time of the nanowire is approximately 15-30 seconds. The power consumption of the wire is now approximately Pwire $=\mathrm{I} 2 * \mathrm{R}$, or $(50 \mu \mathrm{A}) 2 * 8.3 \mathrm{k} \Omega=21 \mu \mathrm{W}$ when biased. 


\section{Measurement Results}

\subsection{Temperature Compensation Introduction}

Measurements were performed for verification of the temperature compensation mechanism using a temperature sensor located close to a single wire of the Pd Chip, hereafter referred to as the 'nearby temperature sensor'. It is anticipated that the temperature response $\tau$ Tns of this temperature sensor may differ significantly from the corresponding temperature time constant of the Pd nanowire, $\tau$ TPd. For the nearby temperature sensor, we choose the SHT15, a calibrated SMT sensor with digital readout.

The sensors were exposed to predefined temperature fluctuations in the signal measurement system. The temperature sequence had a sine profile with a maximum of $28.0^{\circ} \mathrm{C}$ and a minimum of $20.0^{\circ} \mathrm{C}$. The sine profile was chosen in all measurements to ensure both the presence of faster and slower temperature transients. The wire responded to the temperature change as seen in figure 5a. The nanowire response and the nearby temperature sensor output data were plotted. As seen in the chart, the output of the primary nanowire shows an unwanted positive correlation with the temperature change. 


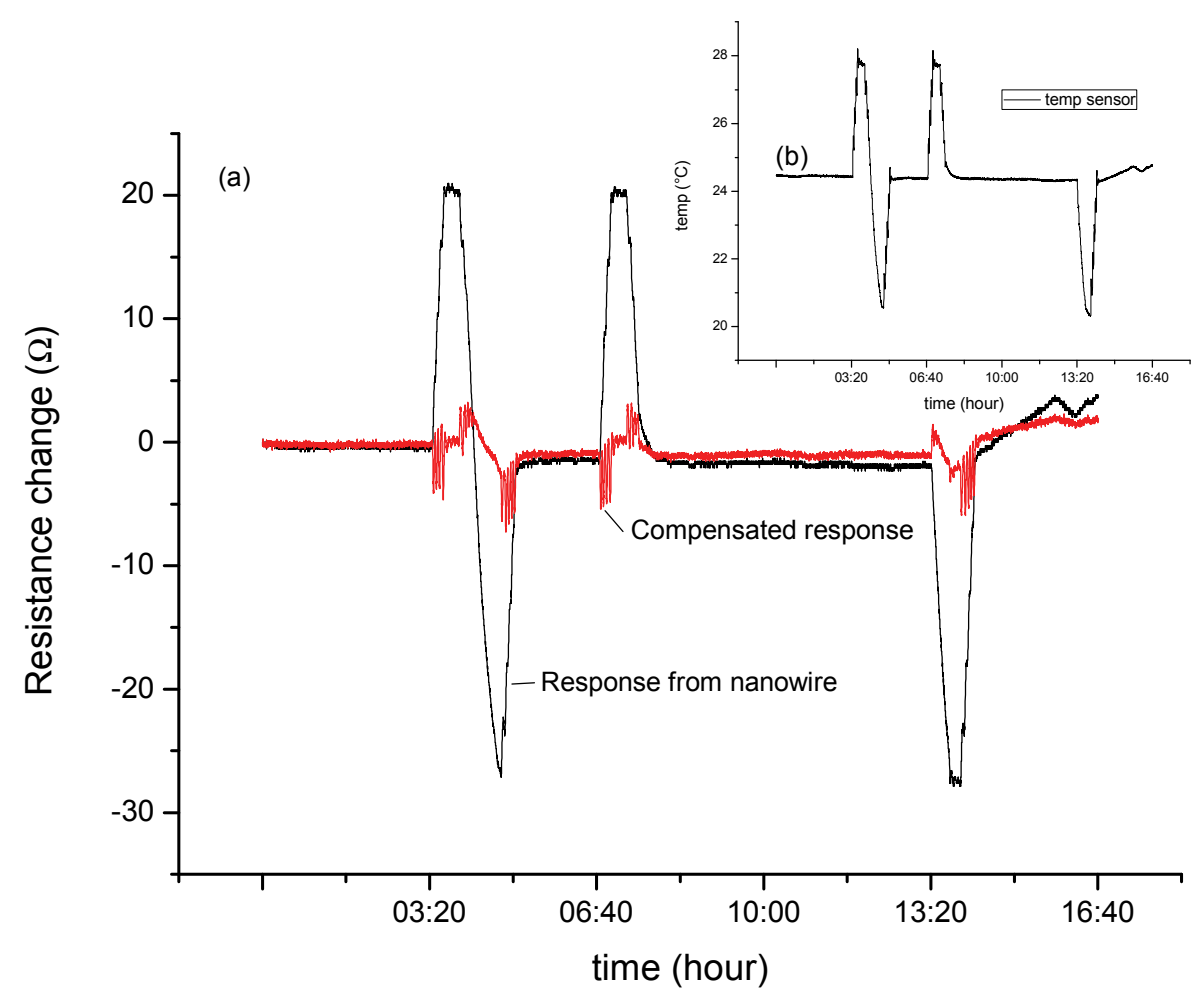

Figure 5.a Temperature sequence with the Pd nanowire response; Figure 5.b Response of the nanowire compensated by the temperature.

Relevant factors include not only the delay in response time but also the exponential time constant $\tau$ of the full temperature response curve. The exponential time constant is defined as the time needed to reach a value of $1 / \exp$ from the final stable baseline $(\mathrm{f}(\mathrm{t})=$ $\exp -t / \tau)$. We observed large differences in these time temperature constants $\tau$ on the order of many seconds for the nearby temperature sensor $\tau$ Tns, and the passivated nanowire $\tau$ Tpa. These time temperature constants are dependent on the specific geometry of the temperature sensitive element, its thermal connection to a substrate and the amount of air flow at a given temperature over this geometry. We found a strong temperature dependence both for the mean hydrogen absorption time $\tau$ Hab (to go from a level of 0 ppm to, e.g., 1000 ppm $\mathrm{H}_{2}$ ), as well as the mean hydrogen desorption time $\tau$ Hre (to go from a level of, e.g., $1000 \mathrm{ppm}$ to $0 \mathrm{ppm} \mathrm{H}_{2}$ ). This finding is analysed below. 
Table 1. Relevant exponential time constants $(\tau)$ for different temperature fluctuation steps

\begin{tabular}{lllll} 
Temperature & $\begin{array}{l}\text { Primary Pd } \\
\text { nanowire } \tau T P d(s)\end{array}$ & $\begin{array}{l}\text { Secondary Pd } \\
\text { nanowire } \tau \text { Tpa (s) }\end{array}$ & $\begin{array}{l}\text { H2 sensor } \\
\text { absorption } \\
\text { (bare) }\end{array}$ & $\begin{array}{l}\text { H2 sensor } \\
\text { resorption }\end{array}$ \\
\hline Tolerance & \pm 20 & \pm 20 & \pm 10 & \pm 10 \\
\hline$(25-) 6^{\circ} \mathrm{C}$ & 125 & 125 & 139 & 67 \\
\hline$(25-) 20^{\circ} \mathrm{C}$ & 390 & 400 & 75 & 30 \\
\hline$(25-) 30^{\circ} \mathrm{C}$ & 160 & 160 & 53 & 20 \\
\hline$(25-) 50^{\circ} \mathrm{C}$ & 80 & 85 & 49 & 23 \\
\hline
\end{tabular}

Although the fast response time of the nearby temperature sensor is on the order of 1.0 second or less, as stated in the sensor datasheet, we see that temperature effects influence the hydrogen measurements for many seconds. It is clear that any temperature sensor that has a temperature response curve different from the temperature response curve of the Pd nanowire itself should not be used for temperature compensation of the hydrogen sensing nanowire.

\subsection{Temperature Compensation Algorithm Using the Nearby Temperature Sensor}

Table 1 shows that the difference in thermal time constants of the hydrogen sensing nanowire and the nearby temperature sensor is significant. As we see, especially at higher temperatures, the hydrogen response times become smaller than the temperature time constants. A temperature compensation technique is therefore desirable. To correct for the fluctuating temperature, a linear adjustment algorithm was used to compensate the output of the primary sensing nanowire with the output of the passivated secondary nanowire. For effective compensation, the difference between the two signals in terms of magnitude and offset must be amplitude-corrected prior to subtraction. The temperature offset, with respect to the nanowire output, was approximated by dividing the extreme values of the Pd wire response by the extreme values of the temperature response. This is an algorithm (van der Bent, van Rijn, \& Puik, 2010) mathematically represented as Equation (1): 


$$
P d_{-} \text {corrected }_{i}=P d_{i}-\left[\operatorname{Temp}_{\underline{i}}-\left[\frac{1}{i} \sum_{i} \text { Temp }_{i}\right] \frac{\min (P d)-\max (P d)}{\min (T e m p)-\max (T e m p)}\right]
$$

Where $\mathrm{Pd}$ is the palladium nanowire sensor data and $\min (\mathrm{Pd})-\max (\mathrm{Pd}) / \min (\mathrm{Temp})-$ $\max (\mathrm{Temp})$ are embedded functions. The min-max functions must be collected during the sampling process, which merely requires a simple compare and hold routine of the minimum and maximum values of both the temperature and the Pd wire response. This calculation shows the maximum possible corrected response because it uses data from the total runtime. Figure 5a shows the compensated response using Equation (1). Note that the amplitude correction of the temperature sensor is performed only once for the whole data set. When a real-time application with this formula was constructed, a modification had to be made to create a moving average.

For this particular setup with the selected near-chip temperature sensor we can see that a normalized differential detection level of $10 \Omega$, equal to approximately $900 \mathrm{ppm} \mathrm{H}_{2}$, can be achieved. Furthermore, figure 5a shows that the largest fault response is observed where the $\mathrm{d} / \mathrm{dt}$ of the temperature is at maximum. This difference can be explained by different $\tau$ values of the nanowire and the nearby sensor to temperature changes. Further experiments confirmed that the fault response increased when the sample interval decreased.

\subsection{Temperature Compensation Using a Second Nanowire as a Temperature Sensor}

In the previous section, the difference in the temperature time-constant of the nanowire and the near-chip temperature sensor causes difficulties in obtaining sufficient and reliable compensation. The Pd nanowire itself can also be used as a temperature sensor with a matching cross-sensitivity. Conformal geometry and responses of individual nanowires have also been demonstrated (Offermans et al., 2009). One of the Pd nanowires can therefore be exposed directly to hydrogen, whereas the other nanowire is passivated and used solely as a reference temperature sensor. Some experiments used a passivation layer with a thickness of about $400 \mu \mathrm{m}$ using 2-hydroxyethyl methacrylate. The response of the passivated wire to hydrogen can be observed in Figure 7 at 4 h50. 
The same temperature waveform was used in each experiment, and $\mathrm{H}_{2}$ insertion was performed.

When using the secondary nanowire with a passivation layer, it is no longer necessary to handle signal domain differences; the min/max parts of equation 1 can therefore be skipped. A moving average high-pass filter (MAF) is proposed, derived from a standard low-pass MAF (Smith, 1997), for each wire, which can be subtracted to obtain a valid signal response. The mathematic representation of a high pass MAF is as follows:

$H(f)=\left(1-\operatorname{abs}\left(\sin \left(\pi^{*} f^{*} M\right) / M \sin \left(\pi^{*} f\right)\right.\right.$

Where $M$ represents the number of points and $\mathrm{f}$ is the frequency, running between 0 and 0.5. This introduces a high pass cut-off frequency that is dependent on the length of the MAF and the sampling frequency.

This high pass can be rewritten for the two wires, resulting in:

$y[n]=\left[x[n]-\left[\frac{1}{k} \sum_{k} x[k]\right]\right]-\left[z[n]-\left[\frac{1}{m} \sum_{m} z[m]\right]\right]$

In this equation $k$ and $m$ are the number of points, $x$ represents the data from the primary wire and $z$ is the data from the secondary wire. This algorithm was applied to the data from the palladium wires. 

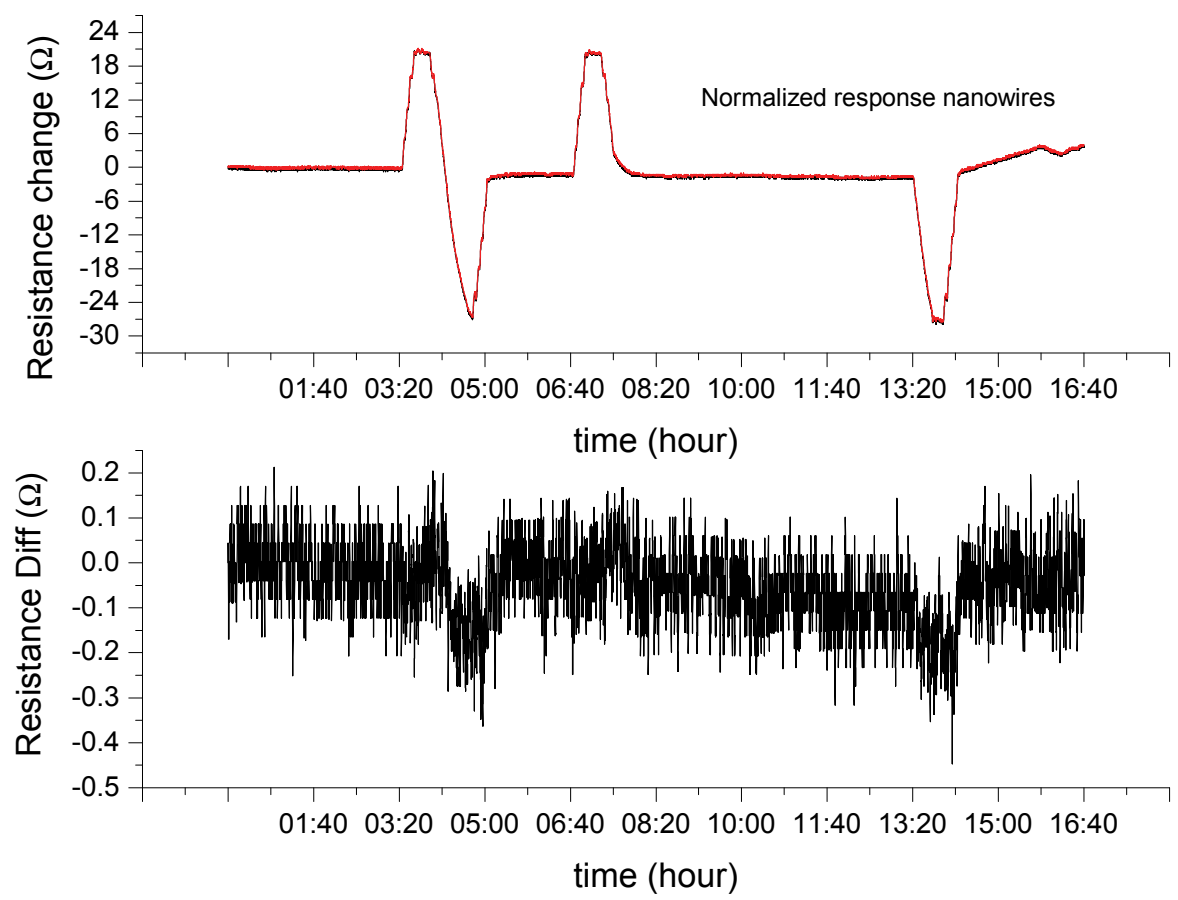

Figure 6. Response of the nanowires only to temperature variations. Upper chart shows temperature sequence with the Pd nanowires responses. Lower chart shows the mutually compensated nanowire response.

In Figure 6, upper chart, the responses of both nanowires are plotted. The lower chart shows the output with the result of Equation 3. This was performed with a 1000-point data buffer $(\mathrm{M}=1000)$. In the absence of $\mathrm{H}_{2}$, we see that the noise base level is less than $0.7 \Omega$, equivalent to $70-80 \mathrm{ppm}_{2}$.

\subsection{Testing the Temperature Compensated System with Hydrogen}

To verify the temperature compensation, the system was tested with hydrogen. Hydrogen was inserted into the signal measurement system up to a level of $1200 \mathrm{ppm}$ while flushing the chamber. At the same time a significant temperature change was initiated. The results are plotted in Figure 7. 


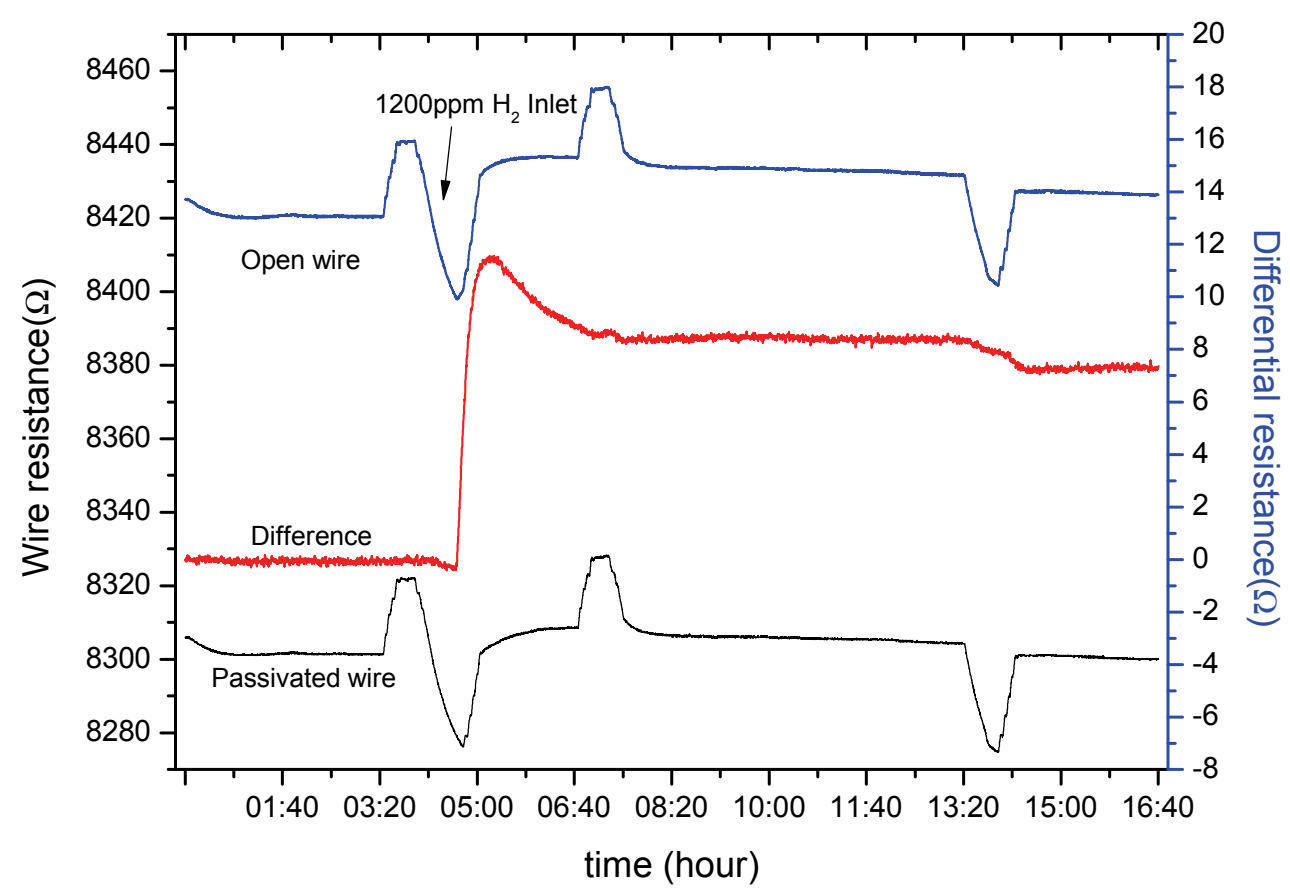

Figure 7. Temperature sequence with the Pd nanowire responses, similar to Figure 6. The arrow shows the input time of $1200 \mathrm{ppm} \mathrm{H}$. The difference chart, in red, shows the compensated nanowire response and $\mathrm{H}_{2}$ detection.

The data of the system combined with Equation 3 showed a full response to the $\mathrm{H}_{2}$ increase, with no noticeable influence from the significant temperature change. When the difference between the time constants of the open wire ( $\tau$ TPd) and the time constant of the compensation wire ( $\tau p a)$, called $(\Delta \tau)$, is smaller than the time constants of absorption ( $\tau \mathrm{Hab}$ ) and desorption ( $\tau \mathrm{Hre}$ ) of the sensing wire, it is possible to adequately compensate for temperature changes.

The time constants of the sensing devices were measured in a forced convection oven with instant heating from approximately $25^{\circ} \mathrm{C}$ to $50{ }^{\circ} \mathrm{C}$. The values of the passivated, non-passivated, and near-substrate temperature sensors were recorded at an interval of $20 \mathrm{~ms}$. For the nanowire, this resulted in a first order effect of $85 \mathrm{~s}$ ( $\tau \mathrm{Tpa}$ ), and for the nearby temperature sensor a first order effect of $43 \mathrm{~s}$ ( $\tau$ Tns). The equality between the $\tau$ of both the passivated and open sensor is excellent and differs by no more than 1 sec after fitting. A first order fit of the nanowires showed a $\tau$ Tpa of $85 \mathrm{~s}$ and $\tau$ TPd $86 \mathrm{~s}$. The 
response time of this $25^{\circ} \mathrm{C}$ temperature step is equal for the passivated wire and the non-passivated wire. The first response of the near-chip temperature sensor was 600 ms slower than the nanowires, indicating a different substrate or configuration of the integrated circuit. Because the system reaches a new balance in the amount of hydrogen in the wire, it is possible to take an absolute measurement after calibration of the hydrogen concentration with the coupled wires. This would involve a translation table that converts the resistance of the wire to an absolute hydrogen value.

\subsection{Early Warning Fast Response}

The section above shows that the absolute concentration of hydrogen can be measured within 10-20 seconds using the temperature compensating algorithm. The absolute concentration is of less concern for an early warning system. Here, the primary interest is detecting a rapid change in the $\mathrm{H}_{2}$ concentration in the event of a major leak, for instance. We have determined the response and recovery times of the Pd nanowire upon direct bulk exposure to hydrogen. We found hydrogen response values of less than 600 ms and a release time of 15 seconds at high concentrations above 2000 ppm (see Figure 8), hydrogen response values of about $900 \mathrm{~ms}$ and a release time of about 25 seconds at a concentration of $200 \mathrm{ppm}$. 


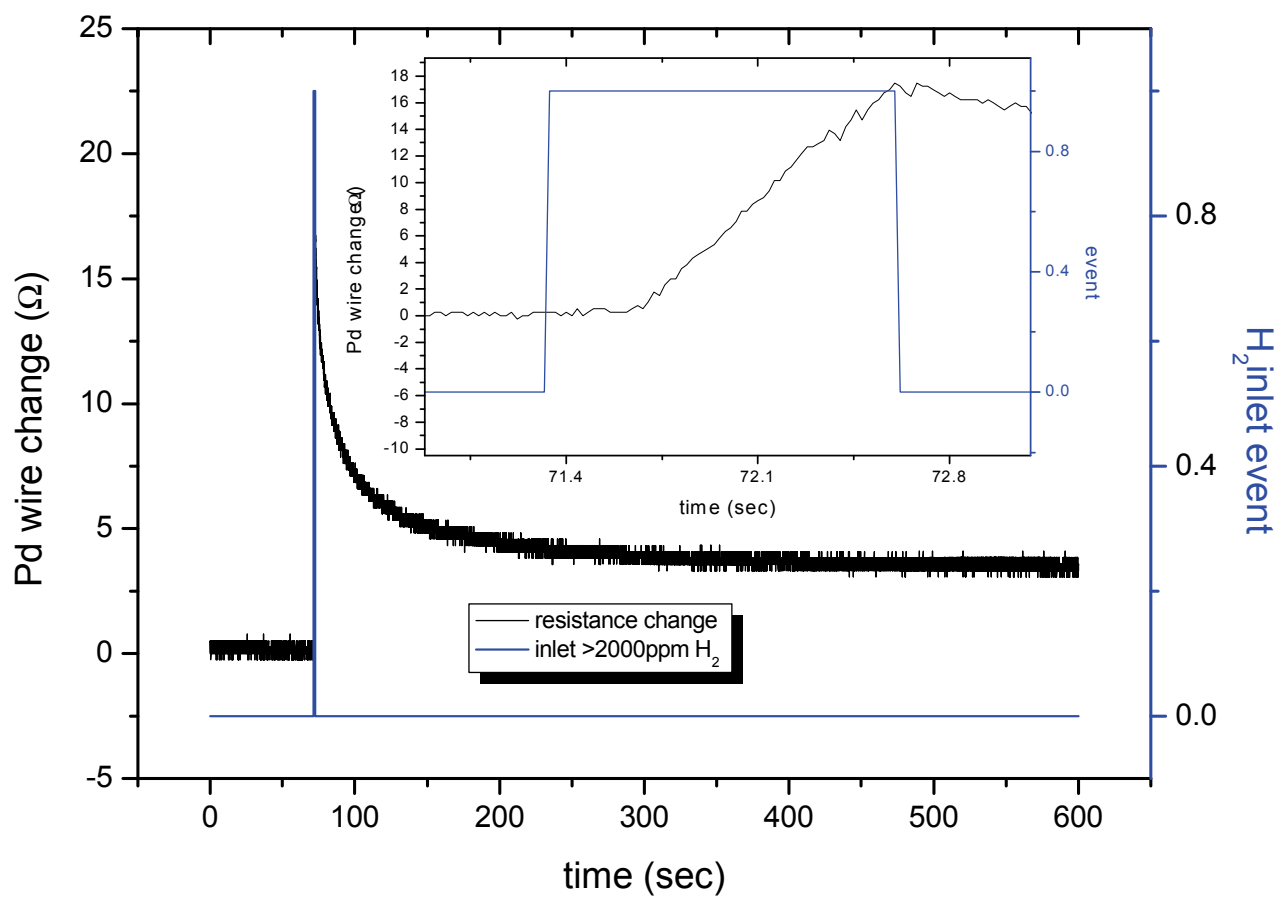

Figure 8. Early response and recovery to an excess of $\mathrm{H}_{2}$. The relative change $\Delta \mathrm{R}$ in resistance of the Pd nanowire is plotted over time at a hydrogen concentration of 2000 $\mathrm{ppm}$. The insert shows a delay in response of about 500-600 ms.

The delayed response time of the near-chip temperature sensor is on the order of 1.0 second, while the delayed response time of the Pd-wires is on the order of $600 \mathrm{~ms}$, while maintaining a perfect $\tau$ match.

\subsection{Discussion}

The precise mechanism of hydrogen detection based on the nanowire geometry used in this paper remains unsolved, but we tentatively propose the following. Molecular hydrogen first dissociates at the palladium surface into elemental hydrogen and then diffuses into the palladium lattice, causing an expansion of the lattice. The conductivity decreases due to a change from the pure Pd phase to the less conductive $\mathrm{PdH}$ (palladium hydride) phase. When the Pd is applied as a thin film the process of hydrogen adsorption and lattice expansion and contraction is found to be reversible without causing 
embrittlement, as is normally the case with thick Pd films. The amorphous phase of Pd (obtained by evaporation at $1 \AA \mathrm{s}-1$ ) may possibly be retained after exposure at varying low and high hydrogen concentrations at room temperature.

In the process described above we attempted to meet all constraints and to ensure precise measurements of the required hydrogen levels by matching all relevant timeconstants, especially the temperature time constants. Table 1 shows that the temperature time constant of the hydrogen sensing nanowire and the nearby temperature sensor may differ substantially, whereas for the passivated nanowire this difference is quite small. For the hydrogen response times we see that, especially at higher temperatures, they become smaller than the temperature time constants. The measured temperature constant depends on the temperature difference of the air and the convection conditions in the oven and of the $\tau$ of the chosen temperature sensor. Even when the air circulation in the oven is stopped there may still be a significant difference in the temperature time constants, depending also on the heat transfer and capacity coefficients of the connected components. The $\tau$ Tpa of the passivated nanowire, on the other hand, was found to be equal to the $\tau$ TPd. Since the wires are on the same substrate with the same response to temperature changes, the temperature crosssensitivity for a wide temperature range could be minimized using the passivated nanowire as a temperature sensor.

We found $\Delta \tau \mathrm{T}$ ( $=\tau \mathrm{Tpa}-\tau \mathrm{TPd}$ ) values typically less than $10 \mathrm{~s}$ as a setting value. If this $\Delta \tau \mathrm{T}$ is smaller than the absorption and resorption time constants of hydrogen in the palladium nanowire we are close to the maximum achievable compensation for the temperature fluctuations, thus guaranteeing optimally fast early detection.

For comparison, when using the nearby temperature sensor for the temperature compensation algorithm, we see differences up to 50-200 s ( $\tau$ Tns - $\tau$ TPd), making it unsuitable in practice for a fast compensation technique.

In the event of extreme fast temperature transients the $\tau$ Tpa and $\tau$ TPd may start to differ for a short interval. This would result in a false $\mathrm{H}_{2}$ alarm. An additional ultra-low power and fast temperature sensor would be a possible solution. This sensor would need to notify the system that only the temperature is increasing and not the $\mathrm{H}_{2}$ value. 
Table 1 shows that this value, derived from fitted data, is not large. The response value is expected to be lower than the $600 \mathrm{~ms}$ measurement interval that we used.

The ability of the passivation layer to prevent the permeation of hydrogen through this layer for a sufficient time is important; we found permeation values of more than 200 minutes depending on the passivation material and the amount of hydrogen. Extending the permeation time through the use of other materials (such as a thin impermeable aluminium film) is better for absolute level detection during permeation but is not critical for early detection.

\subsection{Conclusion}

This chapter presents a temperature balanced, fast and sensitive early hydrogen detection method. A new technique of deposition and etching under an angle (DEA) that utilizes only conventional microfabrication techniques to successfully realize $\mathrm{Pd}$ nanowires inexpensively at a wafer-scale was also successfully implemented. A microchip with two coupled palladium nanowires was tested under varying temperature and hydrogen conditions in a signal measurement system, enabling measurements over a long period. Two Pd nanowires, one of which acts as a hydrogen sensor and the other as a temperature sensor with a matching temperature timeconstant, proved to be the best choice as inputs for an adequate temperature compensation algorithm. After absolute calibration an absolute hydrogen level can be measured on the order of 15 seconds, whereas an early fast response can be detected on the order of $600 \mathrm{~ms}$. A detection limit of $100 \mathrm{ppm}$ of $\mathrm{H}_{2}$ is achievable even during rapid temperature fluctuations. 


\section{Chapter 4}

\section{Improving the Limits of Detection in Potentiometric Sensors ${ }^{2}$}

\section{Overview}

Potentiometric sensors generally respond with a change in electromotive force (EMF) voltage when the temperature changes. This EMF voltage drift is non-linear and this property makes it difficult to compensate for temperature changes using a linear analogue electronics solution or algorithm. Here we propose improving the sensor characteristics by combining the digitized output of two $\mathrm{CO}_{2}$ rubidium silver iodide sensors with a specially designed digital signal process (DSP) algorithm to improve the limits of detection (LoD). Experiments show that this method achieves a 4.5x LoD improvement at temperature variations of $22^{\circ} \mathrm{C}$ over a measurement period of 22 hours. This enables potentiometric sensors to be used in a low-power wireless sensor network (WSN) application such as air quality control. Furthermore, the influence of depletion of the rubidium silver iodide electrolyte layer can be effectively compensated by determining the decay of the active layer according to the Nernst equation. Knowing the function of depletion over time helps to correct the sensor output and thus makes the output value more reliable.

\subsection{Introduction}

The limits of detection of potentiometric sensors are determined by noise during measurements (Bakker \& Pretsch, 2005) and also by the drift of the sensor as a result of the slowly changing ion activity of the cell over time (Bakker \& Pretsch, 2005; K. S. Kim \& Paik, 1975; Roth, Hartinger, Faul, \& Endres, 1996). Low drift and predictability of the output voltage are key factors for a sensor's usability. A change in the temperature at which the potentiometric sensor operates is also an important factor (van der Bent et al., 2015) in determining the LoD. Historically, the use of potentiometric cells operating at high temperatures has been less problematic with respect to drift because the

\footnotetext{
${ }^{2}$ Accepted and published by the journal Measurement Science and Technology; Co-authors: Puik, Tong, van Rijn
} 
temperature is very well controlled. Due to recent improvements in the construction of electrochemical cells and the use of rare earth metals, potentiometric sensors have become available that operate at room temperature with acceptable drift and noise figures (Bakker \& Pretsch, 2005; Obata, Kumazawa, Matsushima, Shimanoe, \& Yamazoe, 2005; Yamazoe, Hisamoto, Miura, \& Kuwata, 1987). The downside is that fluctuations in the room temperature now also influence the sensor's performance. This occurs not only because temperature is a part of the Nernst equation (see eq.(1)), but also because potentiometric sensors tend to seek a slow setting ion activity equilibrium determined by the temperature change, resulting in an unpredictable drift (Bakker \& Pretsch, 2005; Marsal, Centeno, Odriozola, Cornet, \& Morante, 2005).

This study shows how drift in potentiometric sensors operating at room temperature can be substantially reduced. It is demonstrated that temperature is a key element in drift, but knowledge of absolute temperature is not always needed to compensate for the drift (van der Bent et al., 2015). Furthermore, reduction of the overall ion activity over time as a result of exposure to its reduction agent (here $\mathrm{CO}_{2}$ ) makes is harder to predict the drift. This chapter also proposes a method to predict the ion activity by integration of measured activity.

This chapter is organised as follows: first the properties of the potentiometric cell are shown with respect to its response to temperature and $\mathrm{CO}_{2}$. The drift is then compensated by the temperature influence derived from the Nernst equation using a temperature sensor. Two cells are then subjected to the same temperature variation with one being sufficiently sealed, passivating it to $\mathrm{CO}_{2}$. Then, data from the sealed cell and the open cell configuration are combined, resulting, after calculation, in a significant improvement in the LoD of the measurement. An experiment is then performed to predict the decaying amplitude of the potentiometric cell in order to describe more precisely the E0 value of the Nernst equation over time. Finally, the implementation of the proposed algorithm is explained by its integration in a microcontroller unit. 


\subsection{Experimental}

An experimental setup was created to show the behaviour of the potentiometric cell in a nitrogen atmosphere using an 8.3 litre test chamber (see Figure 1). The absolute $\mathrm{CO}_{2}$ concentration was measured by a non-dispersive infrared (NDIR) sensor with a 0$10,000 \mathrm{ppm}$ range (type EE89). The $\mathrm{CO}_{2}$ concentration in the chamber is controlled by Labview with inlet and exhaust valves. The temperature in the chamber is regulated with a Peltier element, a heat sink and a fan in the chamber. Overpressure is controlled with a pressure transducer; if it exceeds $10,000 \mathrm{~Pa}$, the exhaust valve opens until the pressure returns to ambient level.

It is commonly accepted that the behaviour of a potentiometric cell is predicted by the Nernst equation (Bakker \& Pretsch, 2005; Maruyama, Sasaki, \& Saito, 1987). Ideally, the potentiometric cell voltage is determined by the ion activity. The Nernst equation can be used to describe the ideal response of the cell:

$\mathrm{EEMF}=\mathrm{K}-((\mathrm{RT} / \mathrm{zF}) * \ln \mathrm{aI})$

Where EEMF represents the electromotive force or the measured potential at no current, also referred to as Ecell, $\mathrm{K}$ is a constant potential also referred to as E0 or standard cell potential, aI is the sample activity for ion I with charge $\mathrm{z}$, and $\mathrm{R}, \mathrm{T}$, and $\mathrm{F}$ are the gas constant, absolute temperature, and Faraday constant, respectively.

Since the Nernst equation predicts the output voltage of the cell at zero current, a high impedance amplifier is needed to amplify the signals from the cell. For our measurement setup we used commercially available $\mathrm{CO}_{2}$ sensors because of the acceptable reproducibility characteristics of individual Alphasense C02-D1sensors. An amplifier was designed that could measure up to four $\mathrm{CO}_{2}$ sensors simultaneously. After buffering with a 1pA bias current low noise LTC6081 operational amplifier the four differential signals are digitized with a MAX1229, true differential 12 bit analogue-to-digital (A/D) converter (see Figures 2 and 3). Note that figure 3 shows two of the four identical amplifiers and that only four of the available six channels of the MAX1229 were used. Temperature is measured with a pre-calibrated SHT15 chip. 


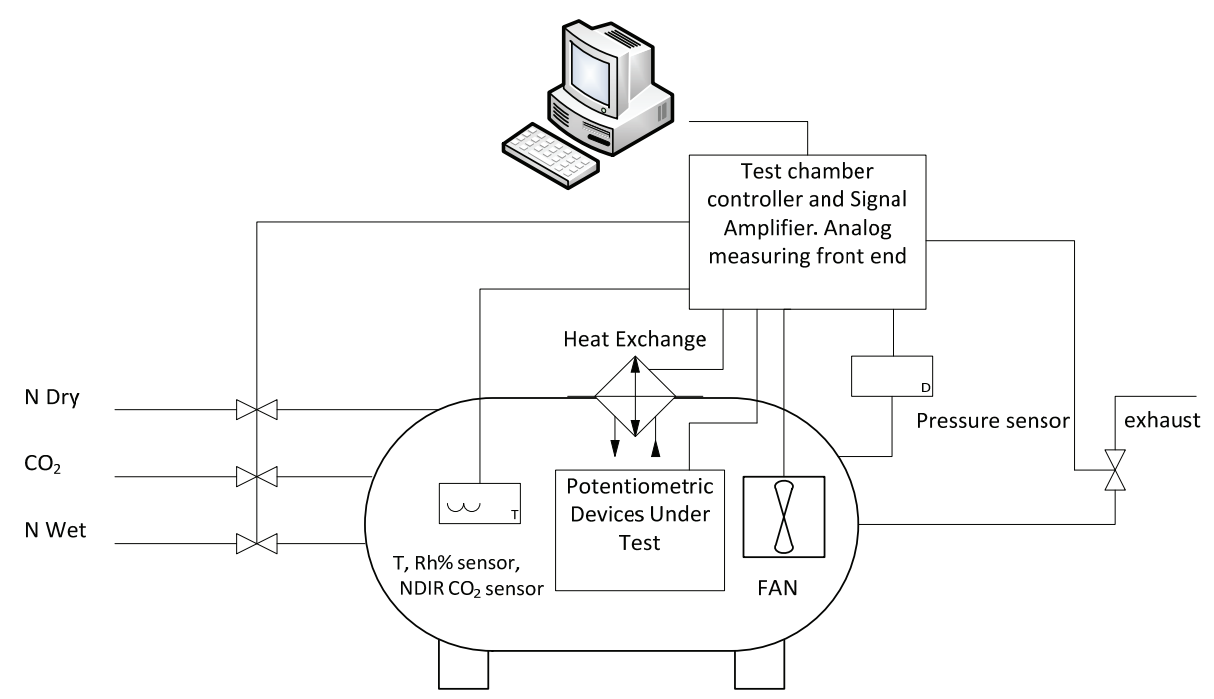

Figure 1. Setup of the measurement environment

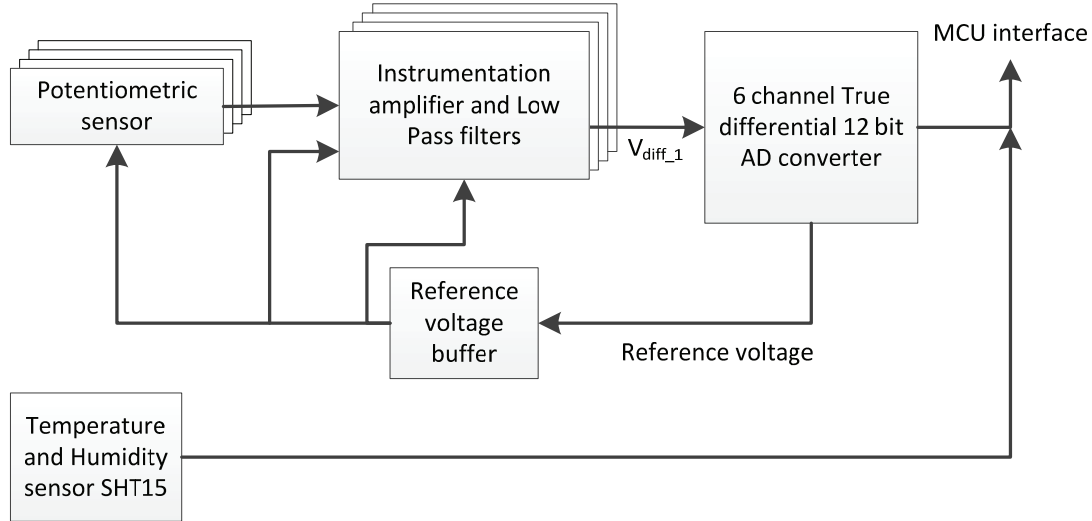

Figure 2. Flowchart of the measuring front end 


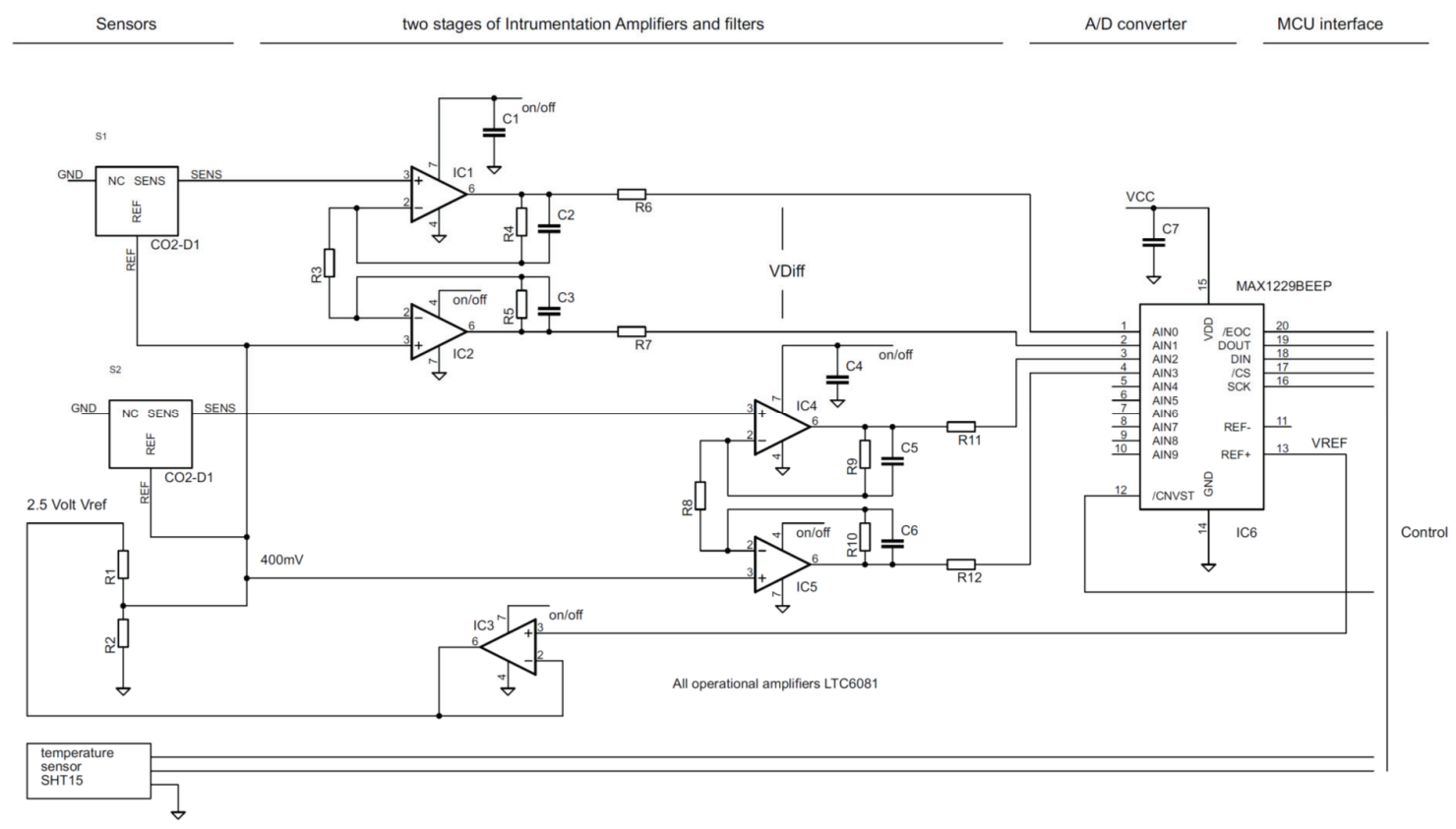

Figure 3. Electronic circuit of the measuring front end

The input resistance of each amplifier is at least $10 \mathrm{G} \Omega$. The bias current needed for the amplifier is kept to a minimum, and this prevents it from interfering with the response time of the sensors. A low bias current also ensures that the baseline voltage variations due to cell-load are minimized (the signal value of the cell voltage returns to the baseline when no $\mathrm{CO}_{2}$ variation is measured). The instrumentation amplifiers are set at an amplification of approximately 40 times. Amplification factors of all operational amplifiers were software-calibrated using a calibrated Agilent 34401a DMM. Using the internal voltage reference of the $\mathrm{AD}$ converter to pre-set the voltage of the cells makes it possible to use the maximum range of the AD converter.

Figure 4 shows the response of the potentiometric cell voltage to $\mathrm{CO}_{2}$ variations from $500 \mathrm{ppm}$ to $2000 \mathrm{ppm}$. During this measurement over 34 hours, the temperature is kept within $0.2^{\circ} \mathrm{C}$, and the relative humidity (\%Rh) is set to $30 \%$. Figure 4 shows representative $\mathrm{CO}_{2}$ responses. The baseline is stable during measurement and continues returning to the baseline value of $10.3 \mathrm{mV}$. 

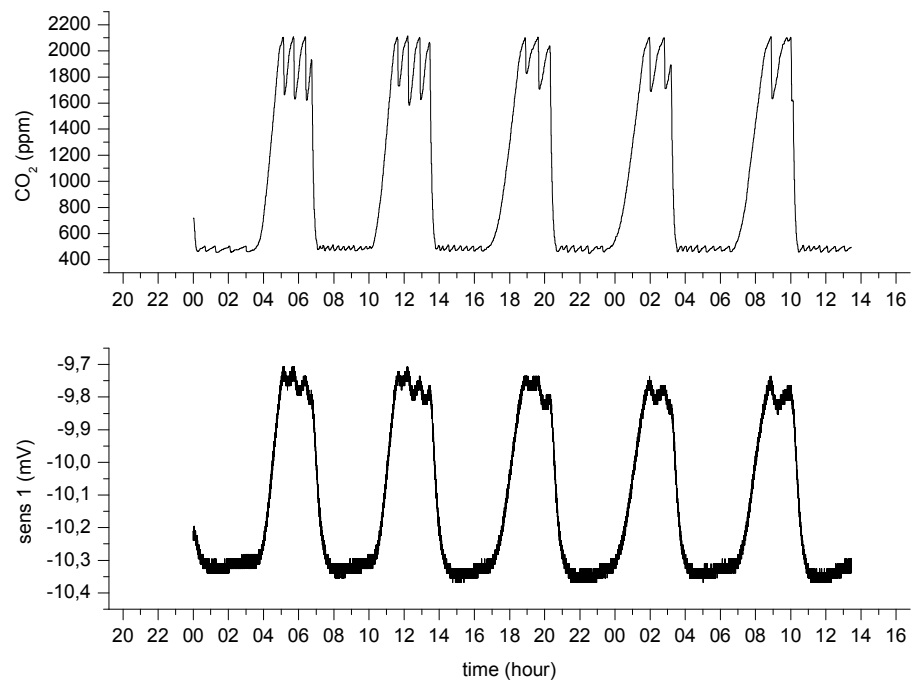

Figure 4. The response of the potentiometric cell voltage to $\mathrm{CO}_{2}$ variations from $500 \mathrm{ppm}$ to $2000 \mathrm{ppm}$

\subsection{Improving the Limit of Detection}

The temperature part of the Nernst equation (Equation 1) has a specific value when the temperature is constant. However, when the temperature changes over time the RT/F part also changes (see Figure 5). 


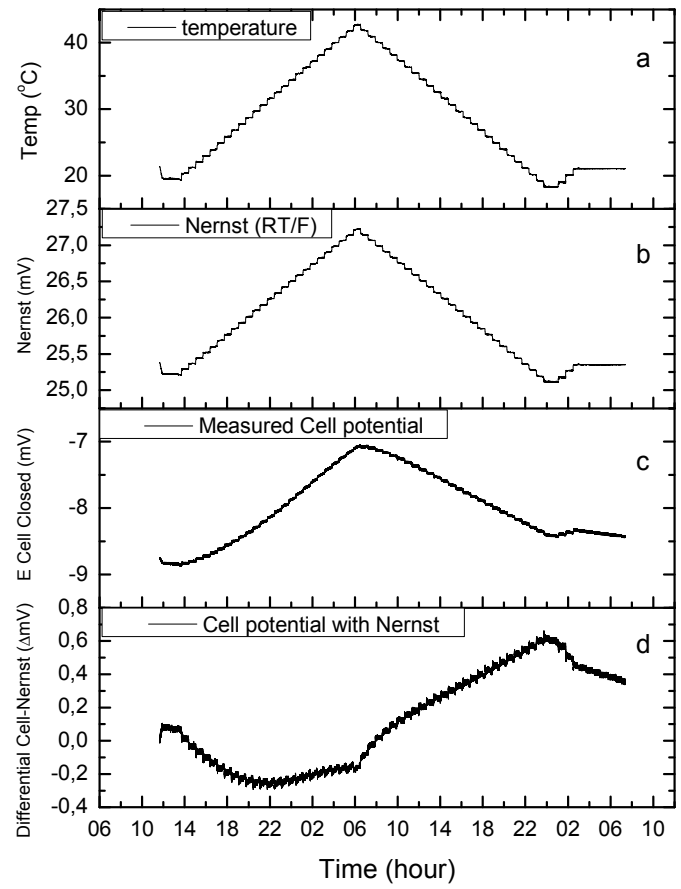

Figure 5. Sensor response: a), b) temperature change according to eq. 1, c) actual measured change, d) differential signal

We tested four individual sensors and selected a pair of sensors with similar output voltages as a function of temperature. Temperature was varied by computer controlled scripts in increments of $2{ }^{\circ} \mathrm{C}$. The temperature is gradually changed from $20^{\circ} \mathrm{C}$ to $42{ }^{\circ} \mathrm{C}$ and then returned to $20^{\circ} \mathrm{C}$. This temperature sequence simulates a considerable variation in temperature, magnifying baseline variations due to temperature variation. The goal of lowering the LoD is to compensate the baseline variation as well as possible. Figure $5 \mathrm{~b}$ shows the response derived from the Nernst equation with the temperature as input in RT/F. Figure 5c shows the actual response of the cell to the temperature change with an output in $\mathrm{mV}$. In figure $5 \mathrm{~d}$ the response of the cell is compensated with RT/F. This improves the LoD by a factor of about 2.5. In the response of two matched sensors, we observe the following: 


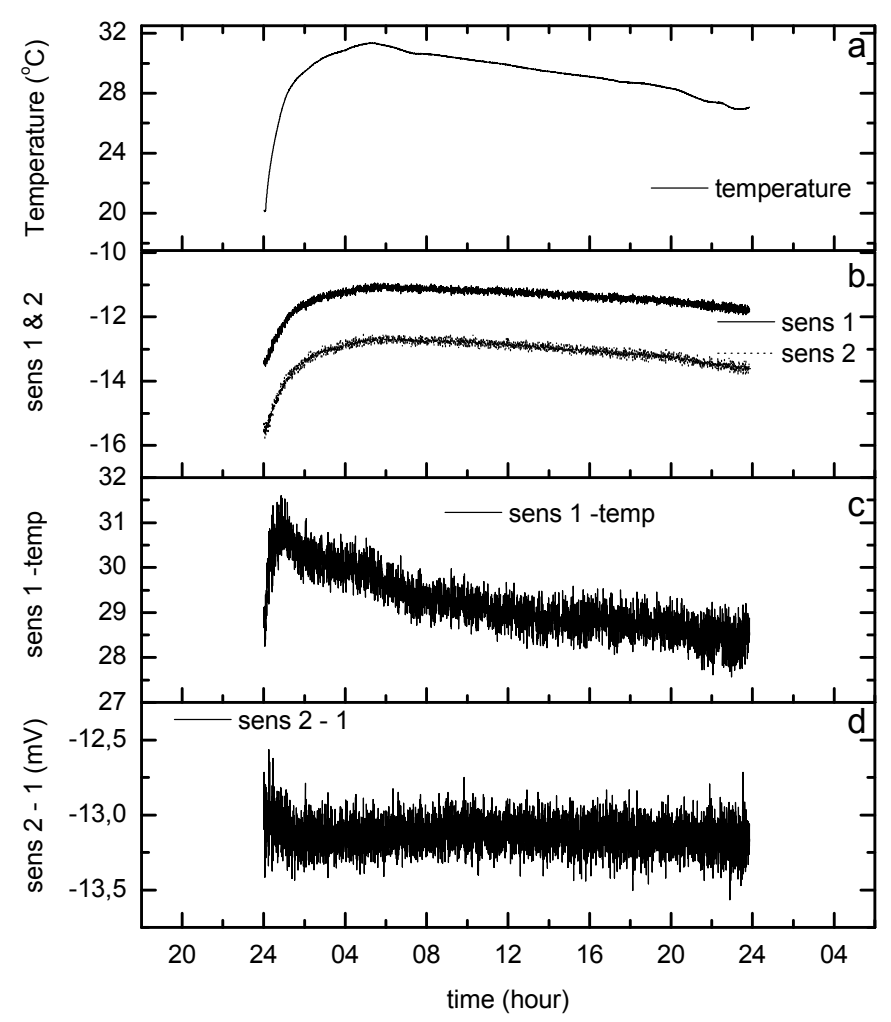

Figure 6. a) temperature, b) sensor response to temperature change for two matched sensors, c) correlation between a) and b), d) differential output signal of two matched sensors

Figure 6 shows the response of sensors when the temperature is varied by $12{ }^{\circ} \mathrm{C}$ over a period of 24 hours. Figure 6 a shows the real measured temperature. Figure $6 \mathrm{~b}$ shows the temperature response of two matched sensors. Visual examination of the data at this point shows a correlation between the sensors and the temperature sensor. The correlation of the data from one sensor and the temperature sensor is plotted in figure 6c; the EMF response, due to temperature variation, may be delayed with respect to the actual temperature. The EMF differential output of the two matched sensors is shown in figure $6 \mathrm{~d}$. Although correlations between the sensor data $(\mathrm{mV})$ and the temperature 
data $\left({ }^{\circ} \mathrm{C}\right)$ are in another domain, we can observe that the relative error in figure $6 \mathrm{c}$ is much greater than the EMF differential output of figure $6 \mathrm{~d}$. There is also a larger error in the response time of the potentiometric sensor compared to the response among the sensors. Decreasing cross-sensitivity to temperature changes with two identical sensors is therefore preferable since it is not easily possible to match the response time of any temperature sensor with the response time of any potentiometric sensor. This seems a good approach to further improve the LoD. In the next experiment, one of the two matched $\mathrm{CO}_{2}$ sensors was sealed, passivating it for $\mathrm{CO}_{2}$. The sealing was performed with a hot glue gun. Both sensors were exposed to the same temperature variation.
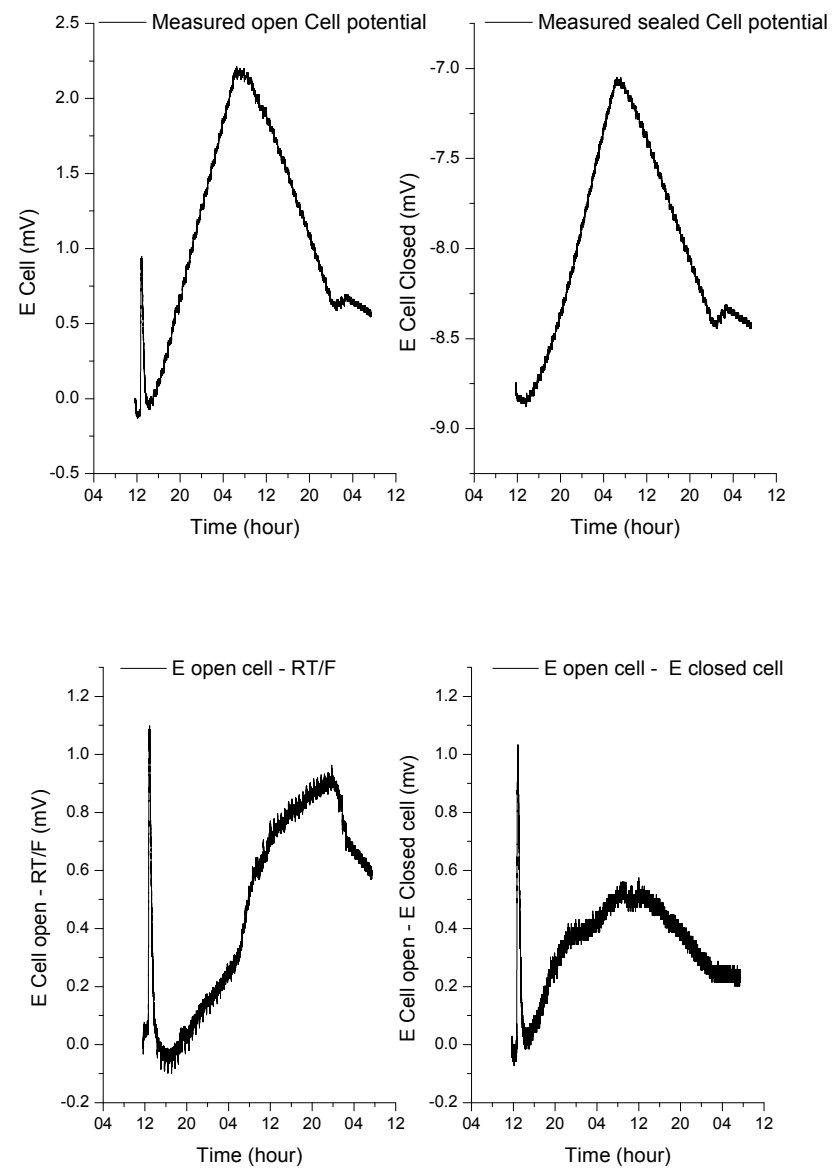

Figure 7. a) Sensor response of open cell with $4000 \mathrm{ppm} \mathrm{CO}_{2}$ followed by a temperature increase of $22^{\circ} \mathrm{C}$; b) Sensor response of sealed cell; c) E open cell - RT/F; d) Differential sensor response

An input of $4000 \mathrm{ppm} \mathrm{CO} 2$ was provided 30 min after the start of the experiment at 12:00 and at $13: 00$ a temperature increase was introduced, from $20^{\circ} \mathrm{C}$ to $42^{\circ} \mathrm{C}$. The 
unsealed sensor (Figure 7a) responds to the $\mathrm{CO}_{2}$ input and the temperature increase, while the sealed sensor (Figure 7b) only responds to the rising temperature. It is worth noting that when we look only at the response of the open cell, we cannot differentiate between the response to $\mathrm{CO}_{2}$ and temperature drift. The sensor's response to $\mathrm{CO}_{2}$ is approximately $1 \mathrm{mV} / 4000 \mathrm{ppm}$ and the cross-sensitivity to the temperature change of $22^{\circ} \mathrm{C}$ causes the sensor to respond by $3 \mathrm{mV}$, or a $\mathrm{CO}_{2}$ equivalent of $12,000 \mathrm{ppm}$. As we see, the response to $4000 \mathrm{ppm}$ of $\mathrm{CO}_{2}$ is 3 times smaller than the temperature response. Figure 7c shows the E open cell - RT/F. When we compensate the open cell signal with the closed cell response (see Figure 7d) we can observe an improvement of the LoD.

\subsection{Prediction of the Reduction of Overall Ion Activity}

Knowing how the sensor will respond within a certain amount of time and after a certain exposure to the detection agent is another important parameter for studying the LoD. Over time, the potentiometric cell will respond with a smaller amplitude change to the same exposure due to depletion of the active layer. Because the front end amplifier could handle four cells simultaneously (see Figure 2), we tested all four of them to gain insight into the depletion characteristics with respect to time and concentration of the analyte, in order to predict the decay of the sensor. The cells were continuously tested in parallel in a controlled environment that was set at $25{ }^{\circ} \mathrm{C}$ with a relative humidity $(\% \mathrm{Rh})$ of $30 \%$. Fixing the temperature at $25^{\circ} \mathrm{C}$, the electromotive force of the Nernst equation becomes:

$$
\text { Ered }=\text { Eo }-((0.05916 \mathrm{~V} / \mathrm{z}) * \ln \mathrm{aI})
$$

We exposed all four cells to a fluctuating amount of $\mathrm{CO}_{2}$ for more than six months, keeping the temperature at $25^{\circ} \mathrm{C}$ with a maximum daily variance of $0.2^{\circ} \mathrm{C}$. The system recorded the values of the potentiometric cell together with humidity, temperature and time. The base level of the $\mathrm{CO}_{2}$ concentration was set at $500 \mathrm{ppm}$ and the top level was set at 6500ppm. During the measurements the response of the cells was recorded in order to obtain the amplitude difference of the cells when exposed from 500ppm to $6500 \mathrm{ppm}$. 
A total of 300 measurements of 14.5 hours each were taken over a period of six months. The data collected from the four sensors was analysed and the results were integrated over time with respect to the total amount of $\mathrm{CO}_{2}$ processed, resulting in Figure 8. We anticipate that the amount of depletion of the active layer is proportional to the $\mathrm{CO}_{2}$ concentration (in ppm) and the time of exposure (in minutes), for which we introduce the unit (ppm*min).

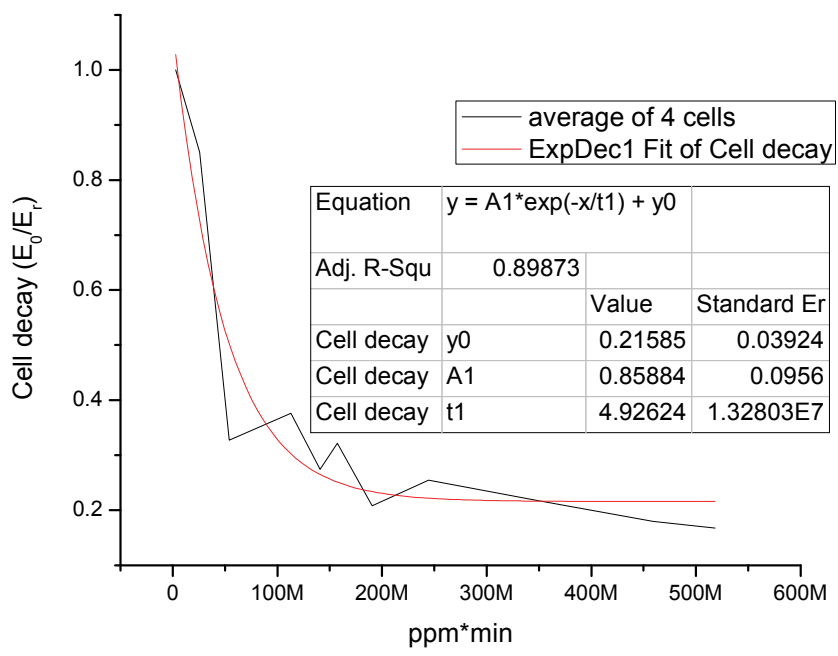

Figure 8. Sensor amplitude decay over time in minutes multiplied by the concentration in ppm

We also anticipate that the amount of depletion plotted against ppm*min will follow an exponential decay curve. The normalized cell decay (E0/Er) is a measure for the maximum output value of the cell. The decay exponent of the sensor can be predicted by integration of the amount of $\mathrm{CO}_{2}$ over time. In this way the $\mathrm{E} 0$ in Equation 2 can be predicted. This means, for example, that when a concentration of $6500 \mathrm{ppm}$ has been measured for a period of 7700 minutes (5.3 days) the amplitude of the sensor would be $60 \%$ of the initial value. The fit contains a residual error. The R-square is approximately 0.9 over the whole range, defining the usability of the fit. The fitted curve shows a maximum error of $20 \%$ of the output value at $50 \mathrm{M}\left(\mathrm{ppm}^{*} \mathrm{~min}\right)$.

With this approximation formula the decay of the sensors can be predicted when the total amount of $\mathrm{CO}_{2}$ measured is stored in a memory and integrated over time. 


\subsection{Implementation Approach of the Algorithm}

The end goal of this study is the use of potentiometric $\mathrm{CO}_{2}$ sensors in a WSN. When a digital approach is used, one must address the following steps: (1) Convert the signals of both the sealed sensor and the $\mathrm{CO}_{2}$ sensor to a digital representation; (2) Acquire a running average value of both signals; (3) Adjust the absolute amplitude of both signals to an equal mean; (4) Subtract the value of the $\mathrm{CO}_{2}$ sensor from the passivated sensor; (5) Integrate the amount of $\mathrm{CO}_{2}$ measured, store and correct it; and (6) Produce the final result with a correction of E0.

The signal must be averaged to discard noise and this involves a delay time dependent on the amount of memory on the MCU. The formal representation of the above would be:

Acorrected $_{i}=\operatorname{correct}_{0} *\left(\right.$ sensor $\left._{i}-\left[\left[\frac{1}{i} \sum_{i} \operatorname{sensor}_{i}\right] \cdot \frac{\min (\text { sensor } 2)-\max (\operatorname{sensor} 2)}{\min (\operatorname{sensor} 1)-\max (\operatorname{sensor} 1)}\right]\right)$

Where $\min ()$ and $\max ()$ are functions that hold the minimum and the maximum values of the sensor output. These are needed in order to calculate the mean value of each sensor. The summation part is the low pass filter in digital representation. When the drift reduction length is set by " $\mathrm{i}$ " the total run-time length of the filter is set by the sampling interval and the available amount of volatile storage in the MCU. The total amount of measured $\mathrm{CO}_{2}$ and the time from a real-time clock (RTC) can be stored in writable non-volatile memory.

\subsection{Discussion}

Using a high impedance differential amplifier, we gained insight into the behaviour of rubidium silver iodide-based potentiometric sensors and improved the LoD. The behaviour is predicted by the Nernst equation. Although the trend and response to temperature variations have similarities, the absolute value depends on the initial standard half-cell reduction potential. This means that the absolute drift value due to temperature fluctuations of the individual sensor cannot be used in a direct analogue approach, and an MCU is needed. Direct analogue compensation would only be possible if the individual decay rate or depletion of each sensor and its response to temperature 
variations were equal. If the depletion among sensors were equal in every aspect, analogue calibration of the sensor output would still be a challenge because of the drifting and diminishing output value over time.

The response of the sensor passivated for $\mathrm{CO}_{2}$ continues to follow the expected $\mathrm{RT} / \mathrm{F}$ drift due to temperature variations. The absolute value of this response differs from sensor to sensor.

The digital approach to compensation using an MCU, defined as a 'smart sensor solution', has the advantage of handling offset values and signal amplitude differences in the process, as well as the decay of the sensors over time.

The issue of absolute calibration remains problematic. As stated earlier, the 'depletion' of the potentiometric cell causes the cell to become less sensitive to $\mathrm{CO}_{2}$ variations, resulting in an amplitude error. This means that the absolute response to $\mathrm{CO}_{2}$ predictably decreases over time when the total amount of $\mathrm{CO}_{2}$ measured over time is integrated. By doing so, we can predict the E0 of the potentiometric cell and compensate for the error of the detection amplitude. The $\mathrm{CO}_{2}$ smart sensor needs an absolute reference for calibration purposes when drift values exceed the allowed maximum. This user-chosen maximum drift is set by the specific application.

Since this sensor is to be integrated in a WSN used in buildings, it is not unlikely that a central $\mathrm{CO}_{2}$ sensor would transmit an overnight reference value throughout the wireless network. This reference could be a wired, centrally placed, NDIR sensor in order to provide a calibration value.

\subsection{Conclusion}

Potentiometric sensors operating at room temperature have significant potential for measuring $\mathrm{CO}_{2}$ levels with a low power demand in wireless sensor systems, provided that adequate temperature compensation is available when the room temperature fluctuates. The EMF voltage drift of a potentiometric sensor with respect to temperature is a non-linear function, and this study shows that it is possible to achieve temperature compensation using an extra sensor as a reference to reduce the temperature drift, thereby improving the LoD. The outputs of two potentiometric sensors can be digitized and amplified using a high impedance differential amplifier, and it is demonstrated that 
a combination of responses can be used in a digital signal process to improve stability and accuracy when E0 is known. This work enables potentiometric sensors to be used in environments such as schools and office buildings to monitor $\mathrm{CO}_{2}$ levels with a WSN and contribute to better air quality. 


\section{Chapter 5}

\section{Improving the Limits of Detection in Capacitive Sensors ${ }^{3}$}

\section{Overview}

Accuracy in capacitive sensing is determined not only by the sensitivity of the capacitive sensing element but also by the physical measurement method used. Here we show that the limits of detection can be improved when a nano-scale capacitive sensor used at room temperature is brought into close proximity with a custom-built capacitance interface. Specifically, the response of an interdigitated electrode (IDE) capacitor with a thin graphene oxide (GO) layer is used to demonstrate an increased response to humidity changes with sub-femtofarad resolutions, thus improving the limits of detection. The response of various IDE structures were also tested with a polymer layer sensitive to acetone gas. We show that the signal to noise ratio is improved when an IDE with a larger total capacitance is used. It is also shown that the GO layer responds with a $1.1 \mathrm{pF}$ capacitance change when the relative humidity is changed by $2 \%$. We further compared the improved response of a micro- versus a nano-gap IDE using an acetone sensitive Poly (4-vinylphenol) (PVPH) polymer layer. The nano-gap IDE shows an increment of the signal output of the IDEs by a factor of 12 compared to micro-gap IDE, while the percentage of change remains equal $(0.2 \%$ at $1000 \mathrm{ppm}$ acetone $)$.

\subsection{Introduction}

Capacitive sensors have been often used for humidity detection (Zaretsky, Melcher, \& Cooke, 1988) and sensing of organic chemical vapours (Hagleitner et al., 2001; Zhou, Hierlemann, Weimar, \& Göpel, 1996). Semiconductor companies have developed several techniques for accurately measuring capacitance changes to control electrical circuits,

\footnotetext{
${ }^{3}$ submitted to the journal Measurement Science and Technology; Co-authors: Puik, Tong, Minh, van Rijn
} 
for example, in the electronic tablet industry and also in the field of contactless capacitive level sensing. There are now state-of-the-art signal processing frontend semiconductor chips that are able to measure in the fF range. A disadvantage with most of these devices is that they do not have the capability to sense in the $\mathrm{fF}$ range with a $\mathrm{nF}$ to $\mathrm{pF}$ bare IDE capacitance, here defined as the overall value of the capacitor including parasitic capacitances. The change of the overall capacitance, or $\Delta \mathrm{C}$, is the changing capacitance fraction.

This chapter is organised as follows: Section 2 provides background regarding the required measurement environment of the IDE chip. Section 3 introduces the signal measurement system needed for capacitance measurement, followed by a cable measurement characterizing its influence. A demonstration of the capacitive noise figure of the setup using an IDE nano-structure capacitor is then presented. Then, the first IDE chip is prepared with graphene oxide and subjected to a test sequence to demonstrate the capabilities of the chosen approach. The next step discussed is a change of the sensing layer on top of the IDE and a nano-scaled structure of the IDE, yielding an increased bare IDE capacitance in order to detect acetone using the same capacitance measurement interface to demonstrate the improved signal amplitude with higher bare IDE capacitance and smaller IDE structures. Finally, section 5 discusses the findings, followed by the conclusions in section 6 .

\subsection{Background}

The capacitive sensing principle is based on the change of dielectric permittivity due to interaction of an analyte with the dielectric layer (Igreja \& Dias, 2004). The capacitive change caused by an analyte can be detected in a broad range, $0.483 \mathrm{pF} / \% \mathrm{Rh}$ (Y. Kim et al., 2009) with regard to humidity changes, or 45 pf/ $\mu$ l chloroform (Harraz et al., 2014), always relative to the bare IDE capacitance. The normal 3D capacitor geometry, however, with two plates and a dielectricum in between, is not preferred in capacitive sensing, for two reasons. First, the dielectricum needed for sensing must be deposited between the two plates, which can be a complicated process when the distance between the plates is on the order of nanometres. Second, diffusion of the analyte in the dielectric layer can only start through its sidewalls and significant time is required before equilibrium can be reached. 
There are a number of strategies that can be followed in order to increase the limits of detection in capacitive sensing. First, when the bare IDE capacitance, C_IDE (see Figure 2 ), is increased, the $\Delta \mathrm{C}$ also changes, so that the signal is larger. With this enlargement of the $\Delta \mathrm{C}$, the signal to noise ratio $(\mathrm{S} / \mathrm{N})$ improves with respect to signal power levels and noise added by the measurement system. Still, in order to be measurable, the $\Delta \mathrm{C}$ needs to be a factor higher than the parasitic contribution. A second factor is the cabling of the measurement setup itself, which can introduce changes in parasitic capacitance values. A third influence is the measurement method, and a fourth measure is effect on the dielectric constant caused by the analyte on the sensing layer. This last measure is always aimed for but hard to implement because the response of the layer to the analyte is difficult to predict. Due to the previously discussed problems with a $3 \mathrm{~d}$ capacitor structure, a simpler two-dimensional structure in the form of an interdigitated electrode (IDE) is widely used as a co-planar electrode substrate (see Figure 1). Detailed calculations for determining the exact bare IDE capacitance of these IDEs have been presented in previous studies (Alley, 1970; Hobdell, 1979; Igreja \& Dias, 2004), since the widely accepted calculation models derived three decades ago show increasing deviation with the nano-scale features of the IDE.
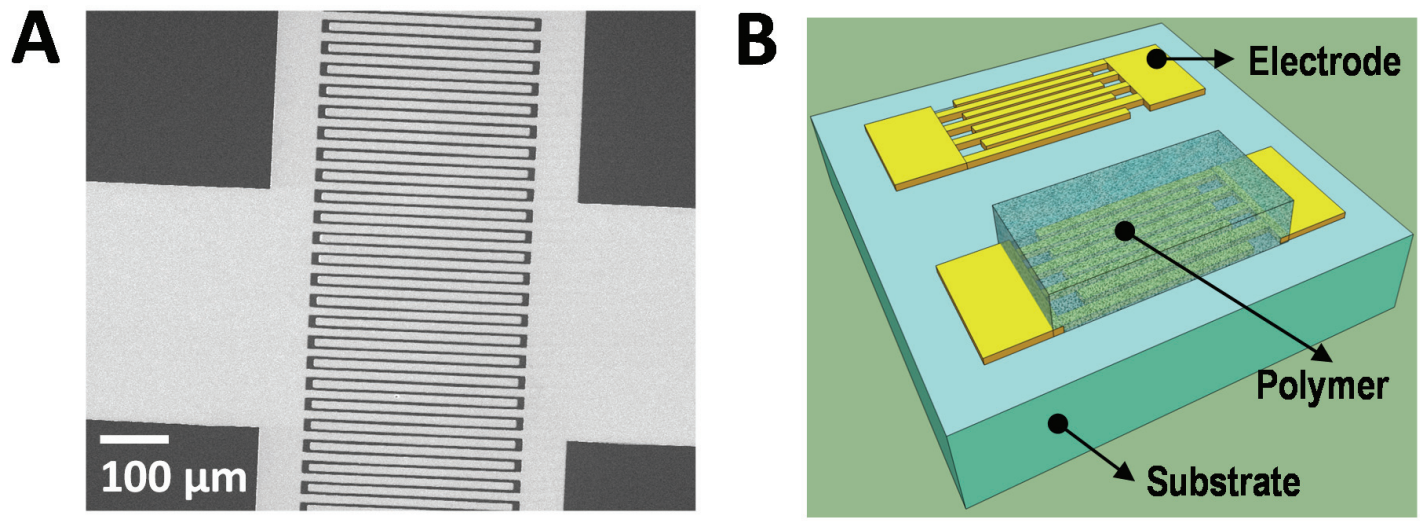

Figure 1. A SEM image of micro-gap IDE (A), and 3D schematic drawing of the IDE with an ideally coated (polymeric) active layer (B).

Nevertheless, optimisation in enlargement of the capacitance within the boundary of the usable detection area on the IDE using nano-structures has indeed greatly improved the bare IDE capacitance. The first experiment performed in this chapter used a custombuilt nano-gap IDE with a comb design and a contact distance of $440 \mathrm{~nm}$. Optimizing the 
IDE structure to a nano-scale and thus increasing the capacitance provides the highest possible front-end gain. If the $\Delta \mathrm{C}$ can be brought into the range of 10 to $100 \mathrm{pF}$, cable interference will be negligible, as shown Section 3. If, on the other hand, the $\Delta \mathrm{C}$ is on the order of 10-1000 fF, changing parasitic cable influences can be problematic. In order to test this capacitance interface, a nano-gap IDE sensor was used with a bare IDE capacitance of 30-50 pF for the graphene oxide humidity measurement. The acetone measurements were taken with a $160 \mathrm{pF}$ bare IDE capacitance on a nano-scale. The limit in constructing these sensors is currently on a nano-scale, which indeed improves the limits of detection (van der Bent et al., 2015; J. Wang et al., 2015). Although further downscaling may be applied to enlarge the bare IDE capacitance by increasing the electrode length, the interdigitated structure becomes fragile when the length of the electrodes increases with short circuits between the electrodes, leading to a low yield of sensor chips.

The limiting factors, summarized in Figure 2, are determined by (1) the bare IDE capacitance of the C_IDE, (2) the thermal and the mechanical stability of the cabling responsible for the parasitic capacitance change during the measuring process with respect to the sensing area of the IDE, or $\Delta \mathrm{C}$, and (3) the resolution of the measurement device. Figure 2 shows a simplified model of the structure shown in Figure 1, connected to a measurement device using a cable.

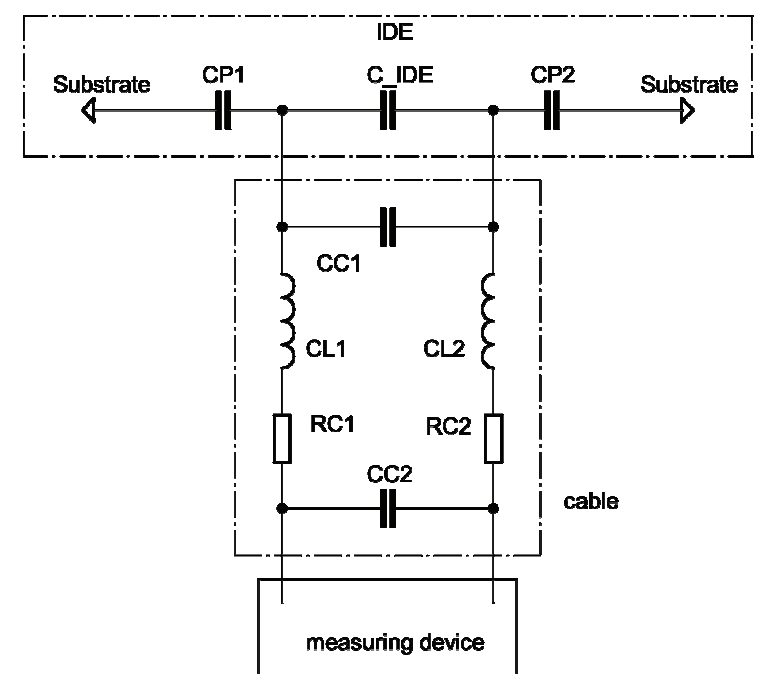

Figure 2. Simplified schematic of resistance, capacitance and inductance present during measurements. 
Figure 2 shows the IDE capacitor formed by the C_IDE, and both parasitic capacitors CP1 and CP2 have a capacitance towards the substrate. The cable needed to connect the IDE to the measuring device consists of a network of resistors, inductors and capacitors (Horowitz \& Hill, 2015). This cabling is a fundamental limitation of the $\Delta \mathrm{C}$ measurement due to temperature variations (Yang \& Yang, 2002), as shown in the experimental setup. Reducing the cable length to zero, and thereby increasing the limits of detection, has led to development of a custom-built capacitance interface.

Since the parasitic capacitances of CP1 and CP2 are stable over time, they can be added as a constant to the bare IDE capacitance. The total bare IDE capacitance, as presented in Figure 2, is equivalent to a parallel circuit consisting of:

Ctotal $=\mathrm{CIDE}+\mathrm{CP}+\mathrm{Ccable}$

\subsection{Experimental Setup}

An experimental setup was created to characterize the behaviour of the nano-scale interdigitated electrode in a clean air atmosphere. The signal measurement system is shown in Figure 3. The humidity in the chamber (8.3 litre), measured with a calibrated AHT-200-01, is controlled by a PC with high-speed inlet and exhaust valves running through, or by passing a bubbler. The humidity sensor was selected to withstand an acetone environment, as used in the second experiment, and not to deteriorate over time. The detection principle of most commercially available humidity sensors is based on a polymer sensing layer. This sensor type is available in factory-calibrated versions with good precision (better than $0.5 \% \mathrm{Rh}$ ) and equipped with a digital interface for easy readout. The downside is that this sensor type will deteriorate over time in the presence of volatile chemicals such as acetone, leading to invalid humidity measurements. The selected humidity sensor, the AHT-200-01 from Ohmic Industries, is a thermal conductivity type. This sensor is capable of measuring in an environment with volatile chemicals such as ketones and hydroxyls. A downside of this sensor type is that it requires temperature compensation of the output data. The temperature in the chamber is regulated with a Peltier element, a heat sink and a fan in the chamber. Overpressure is controlled with an exhaust valve regulated by a pressure transducer. When the internal pressure exceeds ambient levels the exhaust valve opens until the pressure returns to ambient. 


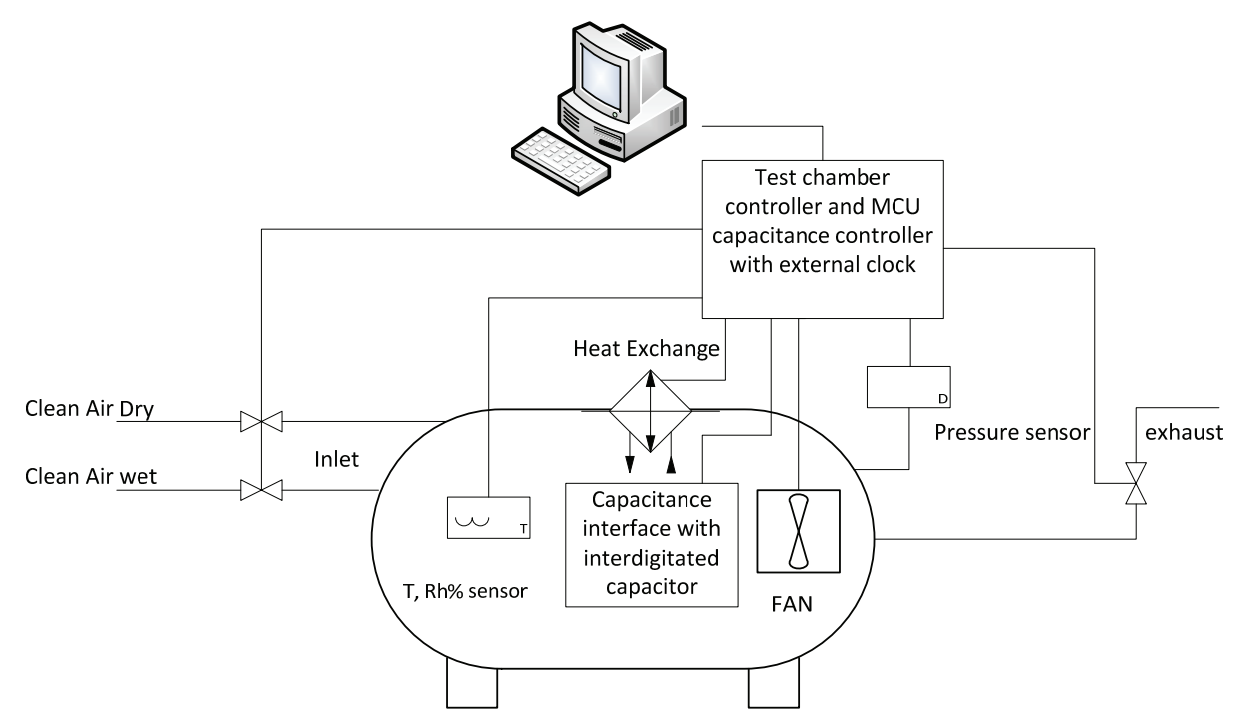

Figure 3. Setup of the signal measurement system for graphene oxide

\subsubsection{Parasitic Capacitance of Cabling}

When a capacitive measurement is taken, the capacitance of the intermediate connection cable can easily change by $100 \mathrm{fF}$ when the cable is moved (see Figure 4), consistent with many other findings (Scharfetter, Hartinger, Hinghofer-Szalkay, \& Hutten, 1998; Yang \& Yang, 2002). When the cable is not moved during the measurement, fluctuations of the capacitance caused by temperature changes may still occur.
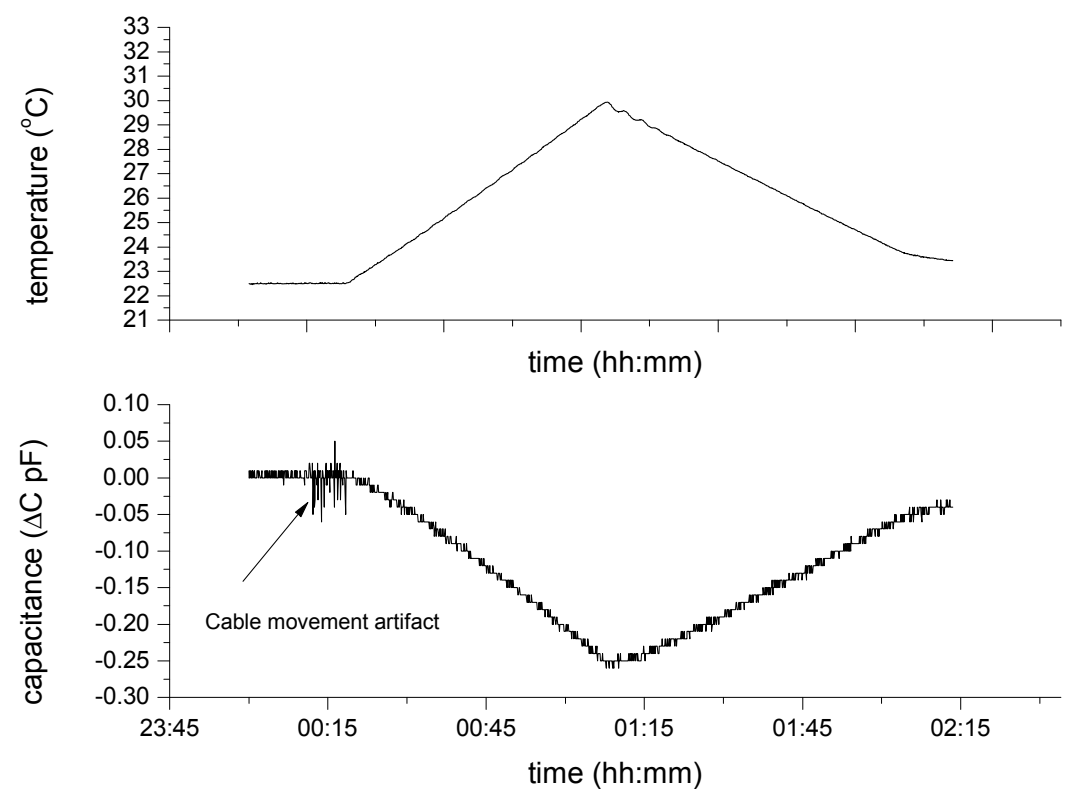

Figure 4. Temperature dependence of one metre RG58 
Figure 4 shows the capacitance change of a standard, 1 metre, RG58, $50 \Omega$ cable towards a temperature change of $8^{\circ} \mathrm{C}$ measured with a calibrated HP4192A impedance analyser after a warmup period of 1 hour at $100 \mathrm{kHz}$. The added capacitance to the sensor from this cable type is approximately $100 \mathrm{pF} / \mathrm{m}$ (Horowitz \& Hill, 2015), and was measured together with the measurement clamp and BNC connector at $126 \mathrm{pF}$.

The limit of detection with regard to the measurement device is determined by the parasitic noise of the cable as a result of mechanical stress and the response of the cabling due to temperature fluctuations in the lab environment. The noise floor, and here also the resolution of the HP4192A, derived from the data in Figure 4, is approximately $20 \mathrm{fF}$. The sensitivity to temperature changes is approximately 31.25 $\mathrm{fF} /{ }^{\circ} \mathrm{C}$ for one metre of cabling, defining the maximum resolution of the single-ended measurement. In order to overcome this fundamental limit of detection, another method is described in the next section.

\subsection{Improving the limits of detection}

Figure 5 shows the flowchart of the capacitance readout interface, which was designed to overcome the cable interference barrier. See Appendix B for detailed information on this interface. The heart of the interface is the FDC2212 semiconductor from Texas Instruments (Texas_instruments, 2015) with a $168 \mathrm{~dB}$ dynamic range comparator resulting in a resolution of $0.3 \mathrm{fF} / \mathrm{bit}$. This semiconductor is capable of handling capacitors up to $250 \mathrm{nF}$, making it suitable for measuring a nano-structure chip with a high (20-200 pF) capacitance. A custom printed circuit board was designed to interconnect the circuits and thereby stabilise the parasitic capacitance to a firm value.

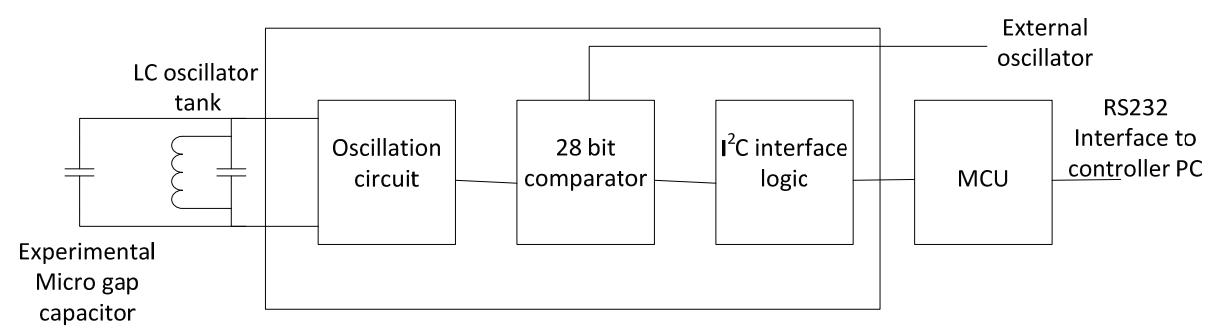

Figure 5. Capacitance interface flowchart 
The double-sided metalized printed circuit board is made of standard FR4 material with gold finish. The data collected and its settings are managed by an ARM7 MCU that interfaces to the chip using the I2C protocol. The controller PC in Figure 3 collects the data from the MCU using the RS232 protocol running over a USB pipe. The FDC2212 is configured to handle an external crystal oscillator of $33 \mathrm{MHz} / 1 \mathrm{ppm}$ that was placed outside the chamber to minimize the temperature variations of the reference. In order to verify the response of the system to humidity changes, the capacitance interface, shown in Figure 6, was placed in the signal measurement system without the nano-gap chip.

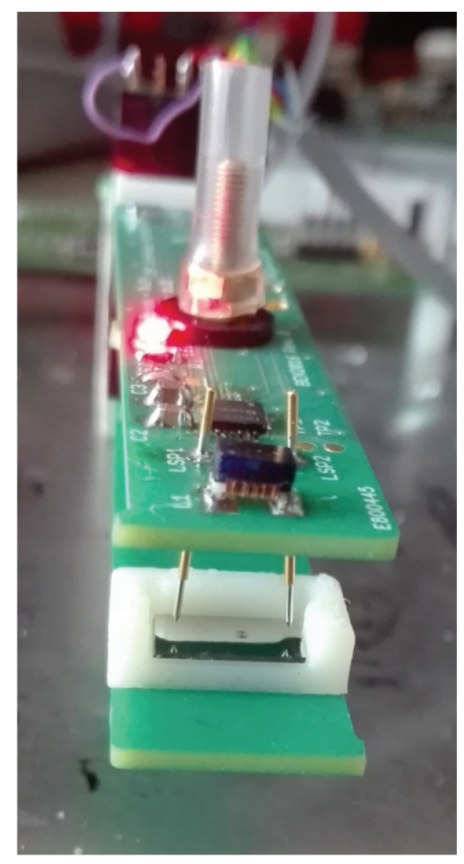

Figure 6. Capacitance interface, front view with nano-gap chip and pogo pins

The humidity was then changed following the predefined script according to the pattern shown in bottom part of Figure 7, between 20 and 75\%Rh with the temperature set at $25^{\circ} \mathrm{C} \pm 0.06$. The data collected by the controller PC was than analysed using Excel and the $\Delta$ capacitance change was calculated and plotted in the top part of Figure 4 . The data shows a maximum variation of $3 \mathrm{fF}$, setting the noise floor of the system. At this time the tank frequency is high compared to other research (Bi et al., 2013), where a maximum of $10 \mathrm{kHz}$ was used. The capacitor used to stabilise the tank is $33 \mathrm{pF}$ with an added parallel parasitic capacitance of $23 \mathrm{pF}$ caused by the printed circuit board routing and the pogo pins. The inductor of the tank is a high-frequency inductor with a value of $18 \mu \mathrm{H}$. The frequency of the tank is determined by equation 1 : 
$F_{\text {res }}=\frac{1}{2 * \pi * \sqrt{(L * C)}}$

Where $\mathrm{L}$ is the inductance in Henry, $\mathrm{C}$ is the capacitance in Farads and Fres is the tank resonance frequency in $\mathrm{Hz}$. At $18 \mu \mathrm{H}$ and $56 \mathrm{pF}$, the tank's resonance frequency is $5 \mathrm{MHz}$. When the nano-gap with GO layer is placed in the tank circuit, the capacitance increases, thereby decreasing the resonance frequency of the tank.
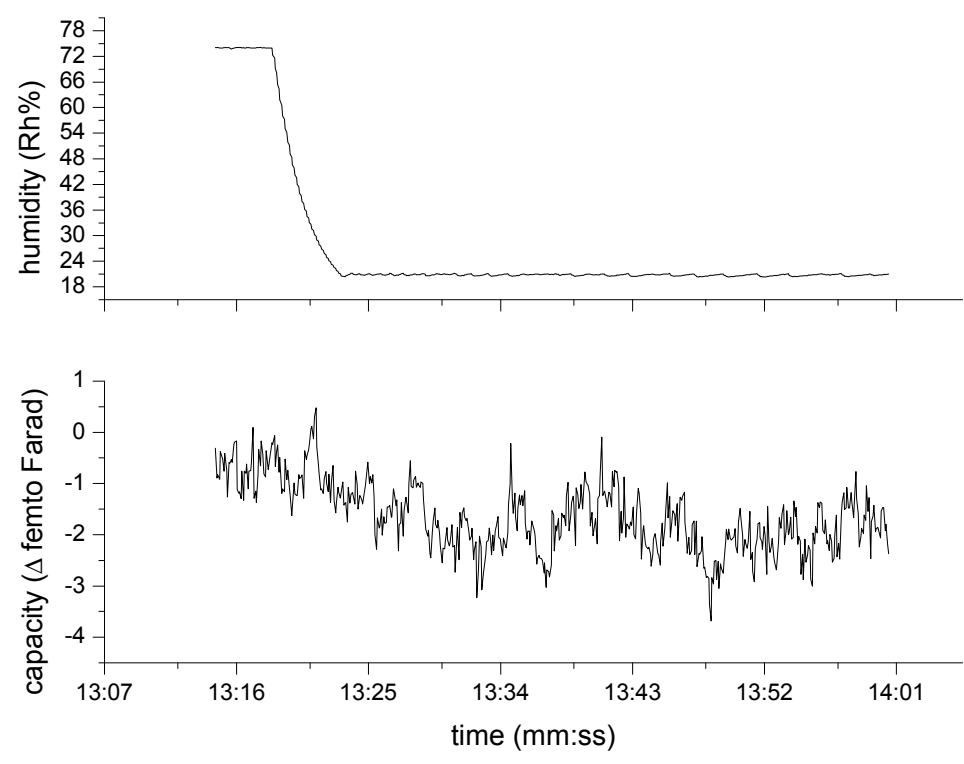

Figure 7. Capacitance interface response to humidity change of $50 \% \mathrm{Rh}$ with an open circuit without IDE, resulting in a $3 \mathrm{fF}$ noise level

After determining the noise floor of the open system (see Figure 7), the nano-gap chip is connected in a parallel circuit to the oscillation tank as shown in figure 5. 

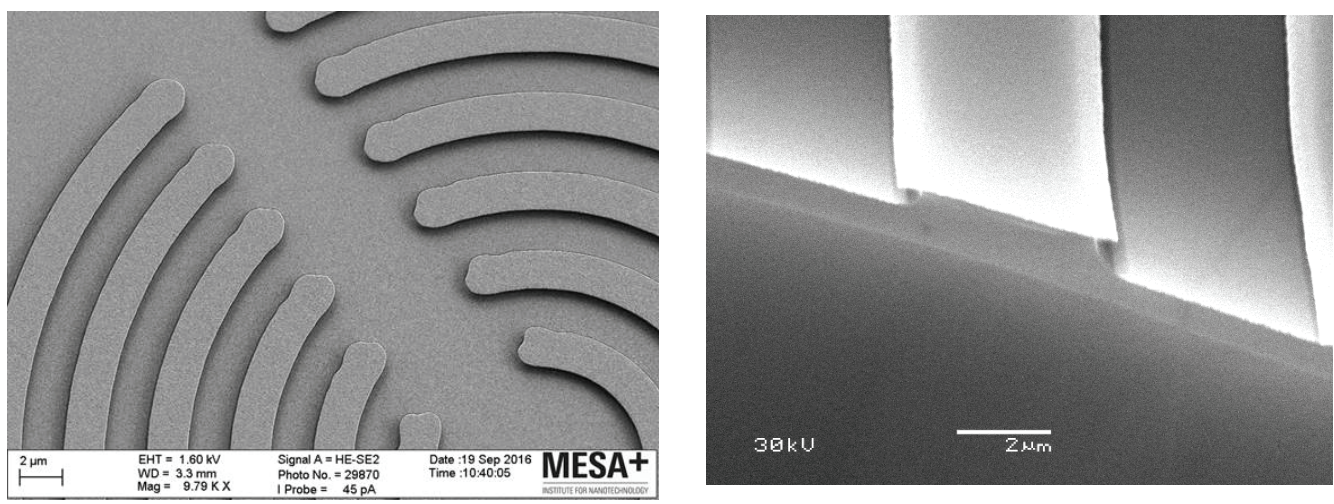

Figure 8. Interdigitated electrode design and SEM close up and cross section of the finger distance of a circular nano-gap IDE 


\subsection{Experiments with the Graphene Oxide IDE ( $36 \mathrm{pF}$ capacitance)}

In the first experiment, the graphene oxide was deposited on top of the chip's sensing area by drop casting. $20 \mathrm{mg}$ of graphene oxide was dissolved in $20 \mathrm{ml}$ purified water and stirred for 3 hours at $25^{\circ} \mathrm{C}$. Three layers of graphene oxide were deposited and each layer was dried in a vacuum at $50^{\circ} \mathrm{C}$ for 60 minutes. The bare IDE capacitance of the clean nano-gap chip was $36 \mathrm{pF}$ with $>20 \mathrm{M} \Omega$ resistance measured with a calibrated Keithley 2000 DMM. After depositing the graphene oxide, the resistance was measured again at $2.15 \mathrm{M} \Omega$. The prepared experimental nano-gap chip was placed in the capacitance interface that connects to the tank circuit using two $0.8 \mathrm{~mm}$ spear tip pogo pins, as shown in Figure 6. Tightening the bolt on top of the printed circuit board results in a lowering of the pogo pins, thereby connecting the nano-gap chip to the resonance tank. After the chip is loaded in the interface, the oscillator tank frequency is now determined by the inductor and a parallel circuit of the capacitor placed on the capacitance interface and the capacitance of the nano-gap sensor with the graphene oxide as dielectricum. After the graphene oxide was deposited on the nano-gap sensor the capacitance increased to $90 \mathrm{pF}$ measured in air at $25^{\circ} \mathrm{C}$ room temperature with an ambient relative humidity of $53 \% \mathrm{Rh}$. The value of the capacitance of the prepared nanogap sensor was measured with a calibrated Agilent 4262A LRC meter at $10 \mathrm{kHz}$. The resonance frequency is now determined by the parallel combined capacitance of $56 \mathrm{pF}$ of the interface itself and the $90 \mathrm{pF}$ of the prepared graphene oxide chip and is approximately $3.1 \mathrm{MHz}$, as verified by measurement with a Tektronix TDS2002. After the chip and capacitance interface were placed in the chamber a triangular humidity change of 55 to $74 \% \mathrm{Rh}$ (see figure 9, middle graph), was programmed while keeping the signal measurement system temperature at $25^{\circ} \mathrm{C} \pm 0.06$. Figure 9 , bottom graph, shows the response of the nano-gap capacitor with GO layer in fF. 


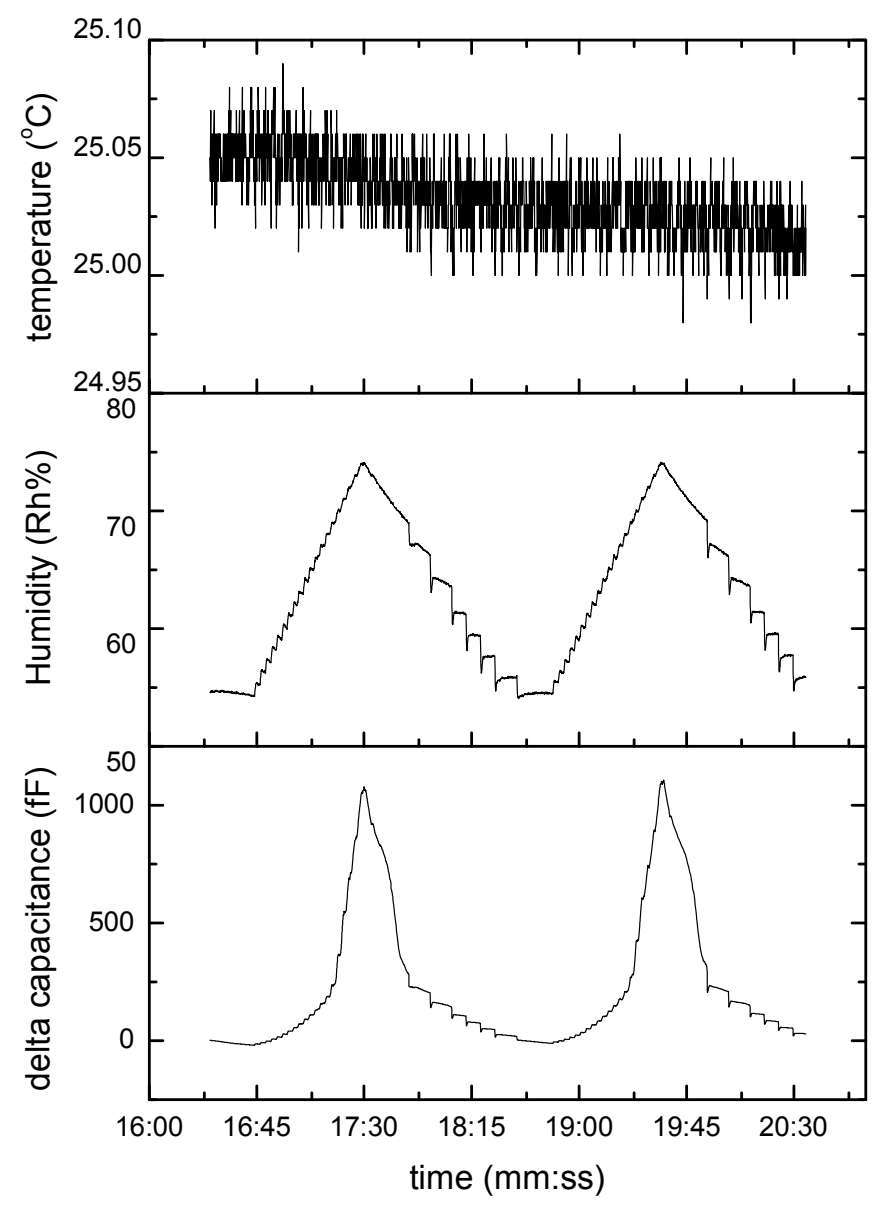

Figure 9. Humidity response of the GO prepared nano-gap sensor

A total measured span of approximately $1100 \mathrm{fF}$ is observed. The resolution of the interface is given in Figure 10, showing a detail of Figure 9 starting at 18:00. 


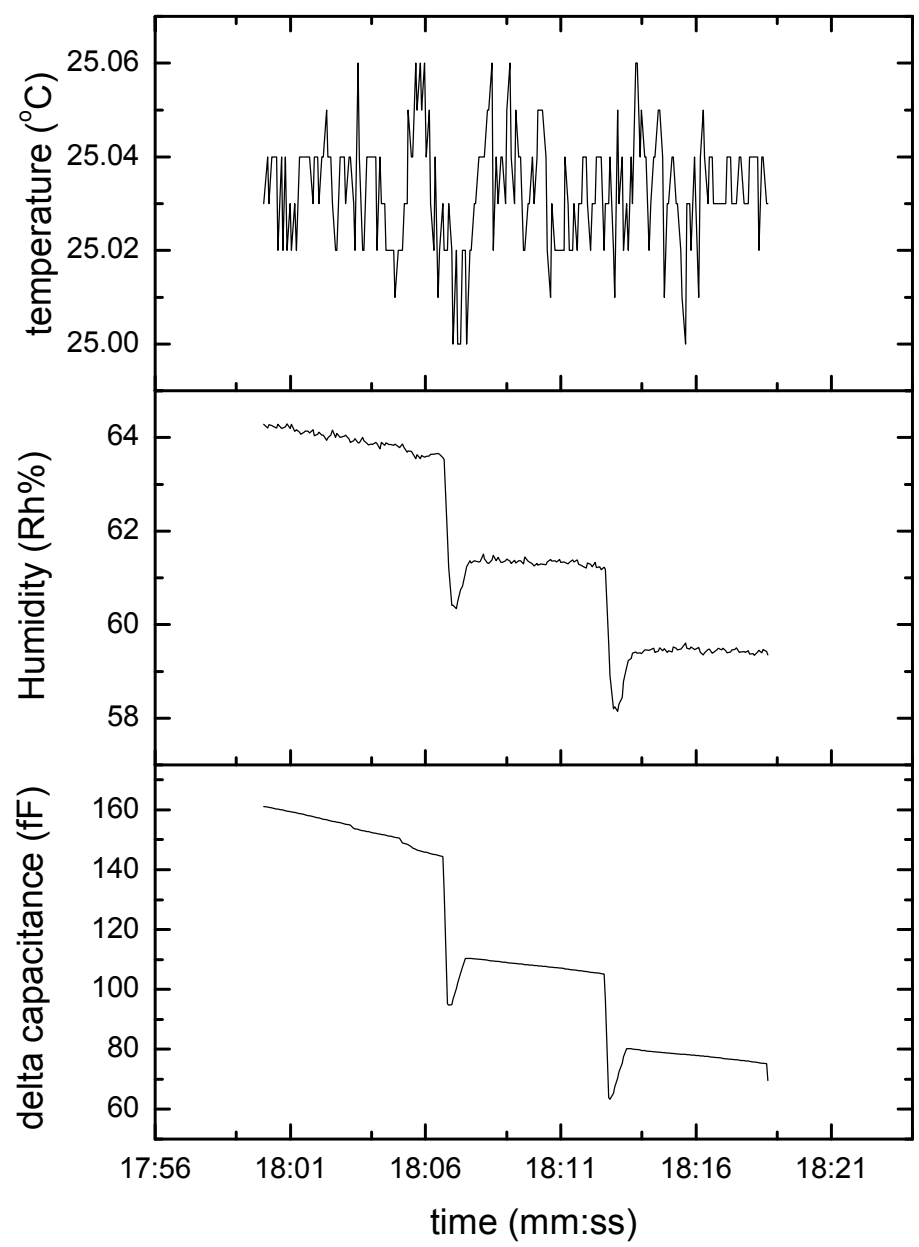

Figure 10. High resolution detail of the measured capacitance in Figure 9

As we see in Figure 9, the response of the graphene oxide to humidity as a dielectricum is nonlinear. This effect was further explored by a nine-fold repetition of the previous experiment. The results from these measurements are shown in Figure 11 with the measured humidity of the reference plotted on the horizontal axis and the capacitance change on the vertical axis. Figure 11 also shows the data fitted to an exponential function with error bars, indicating a 0.97 standard deviation. 


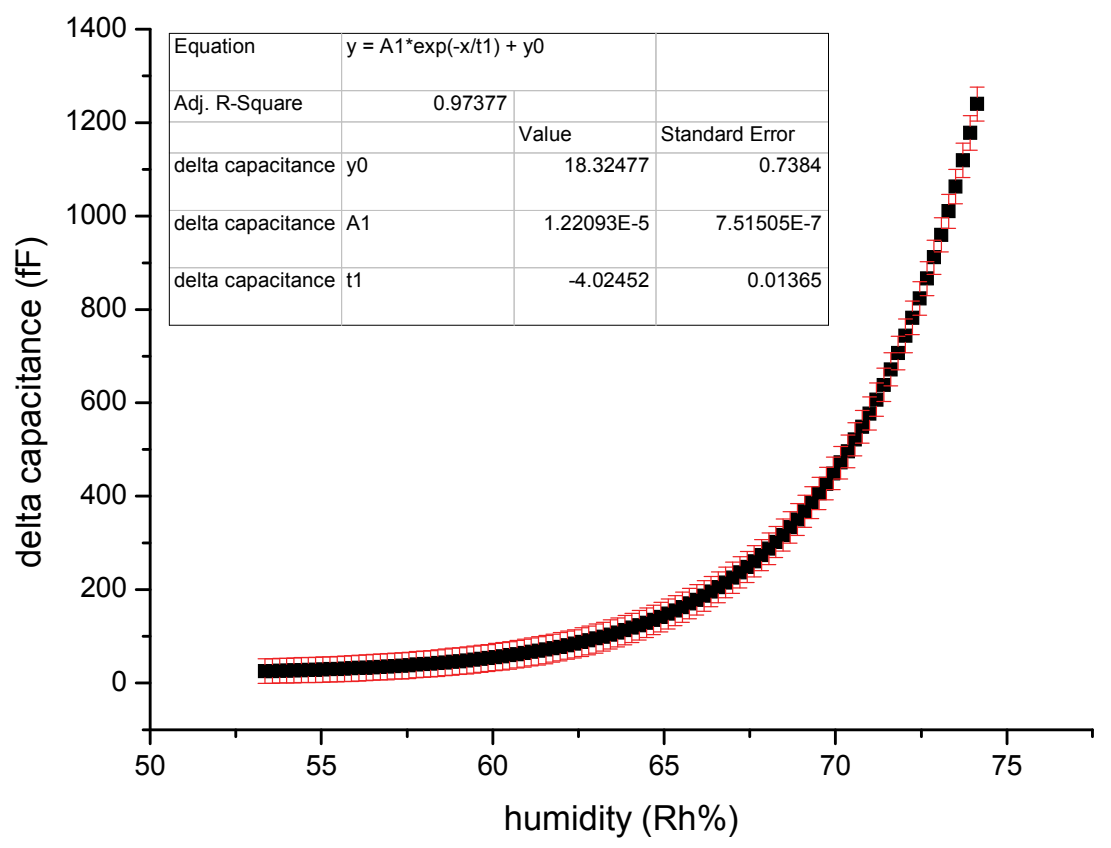

Figure 11. Fitted exponential behaviour of the graphene oxide layer with error bars

The GO measurement shows responses of the material to be on the order of $1.2 \mathrm{pF}$ within the proposed humidity change. When the dielectric change of the sensing layer becomes less and the signal to noise ratio becomes insignificant, while the limit of detection measured with the capacitance interface has also reached the limits of the hardware, improvements can be found in nano-scale features, thereby yielding an increased bare IDE capacitance. In the next experiment, a highly selective polymer layer with a relative low dielectric change was coated on a micro- and nano-scale IDE to gain insight into the detection limit with respect to changing capacitance.

\subsection{Experiments with the Acetone Polymer Layer on the IDE}

In order to control the acetone amount in the signal measurement system, the system described in Chapter 3 was upgraded to accommodate these tests. An additional bubbler with a diluted acetone concentration (1 part acetone to 100 parts demi water) was added to the inlet system, controlled by a high-speed valve as indicated in Figure 12. The concentration in the system was measured using a calibrated PID-AH sensor from Alphasense, capable of sensing 0-6000ppm of acetone. The output of this sensor was fed 
into a calibrated DMM to be read by the control PC. The data from this reference sensor was used in a software feedback loop of the signal measurement system. The script file, necessary for setting values in the system, was enhanced with a line stating the desired concentration of acetone in ppb.

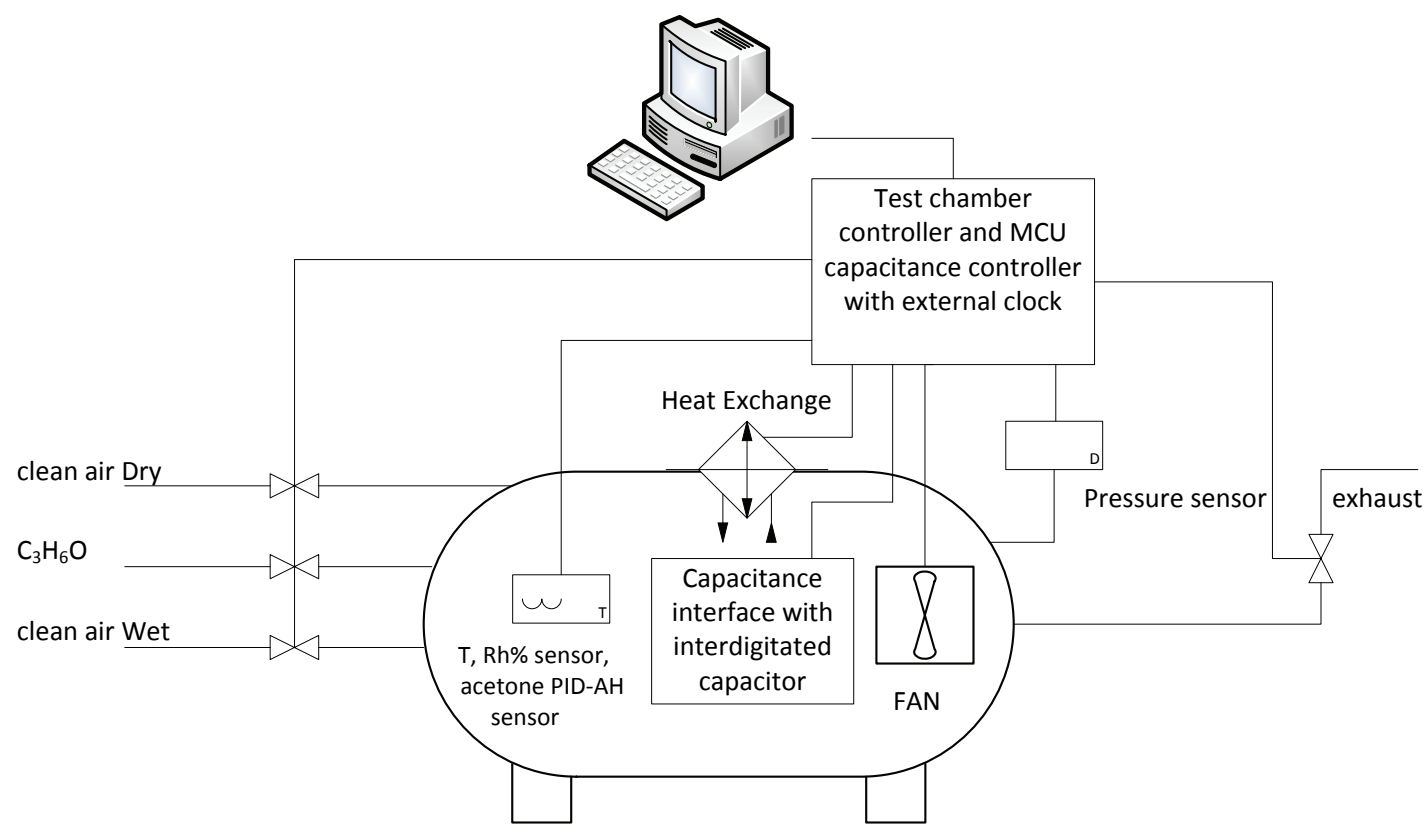

Figure 12. Setup of the signal measurement system for acetone

\subsection{Fabrication of micro-gap interdigitated electrode (IDE) chemo- capacitors}

The micro-gap interdigitated electrodes (IDEs) were fabricated by vapour deposition of $110-120 \mathrm{~nm}$ of gold onto an electrically insulating boron glass substrate. A conventional comb structure with 124 fingers was designed and fabricated on this substrate, with an electrode finger length of $300 \mu \mathrm{m}$, a finger width of $10 \mu \mathrm{m}$ and a gap width of $6 \mu \mathrm{m}$. This design allows the formation of a homogenous polymeric active layer onto the IDE.

Poly(4-vinylphenol) (PVPH) Mw 11,000, and absolute ethanol (98\%) were purchased from Sigma Aldrich. All reagents were used as received. PVPH was dissolved in ethanol by sonication for several minutes until a clear polymer solution was obtained. Microand nano-gap IDE chips were coated with polymer by dipping them slowly into the polymer solution, with the electrodes in a vertical position, until the liquid reached the 
bottom of the electrodes. The chip was held in this position until the gaps between the electrodes were filled with polymer solution by capillary action. This took less than one minute and the filling of the gaps was confirmed by the changing reflection of light. When the gaps had been completely filled, the chip was submerged in the polymer

solution completely, after which the chip was spin coated at $3000 \mathrm{rpm}$ for 2 minutes to form a thin layer. Both nano- and micro-gap IDEs were coated by covering them completely with the polymer solution, followed by the same spin coating procedure. Finally, the samples were annealed in an oven at $60^{\circ} \mathrm{C}$ overnight.

\subsection{Measurements with PVPH Micro- and Nano-gap IDE}

Two different chips were prepared with the same PVPH polymer layer in order to detect acetone with concentration levels up to $1000 \mathrm{ppm}$. Together with the polymer layer, a total bare IDE capacitance of $8.1 \mathrm{pF}$ was measured. Figure 13 shows the response of the sensor to an acetone input in increments of 100-200-400-700-1000ppm over a total time interval of 2 hours. Here we see a response to the acetone input and also, since the polymer is also sensitive to humidity changes, a response to fluctuations in the humidity set at $15.2 \% \mathrm{Rh}$. The temperature was kept stable at $25^{\circ} \mathrm{C} \pm 0.1^{\circ} \mathrm{C}$ during the measurement. The peak value of this micro gap IDE at $1000 \mathrm{ppm}$ acetone is $22 \mathrm{fF}$ while the response to humidity changes of $0.5 \% \mathrm{Rh}$ is no more than $2.5 \mathrm{fF}$. 


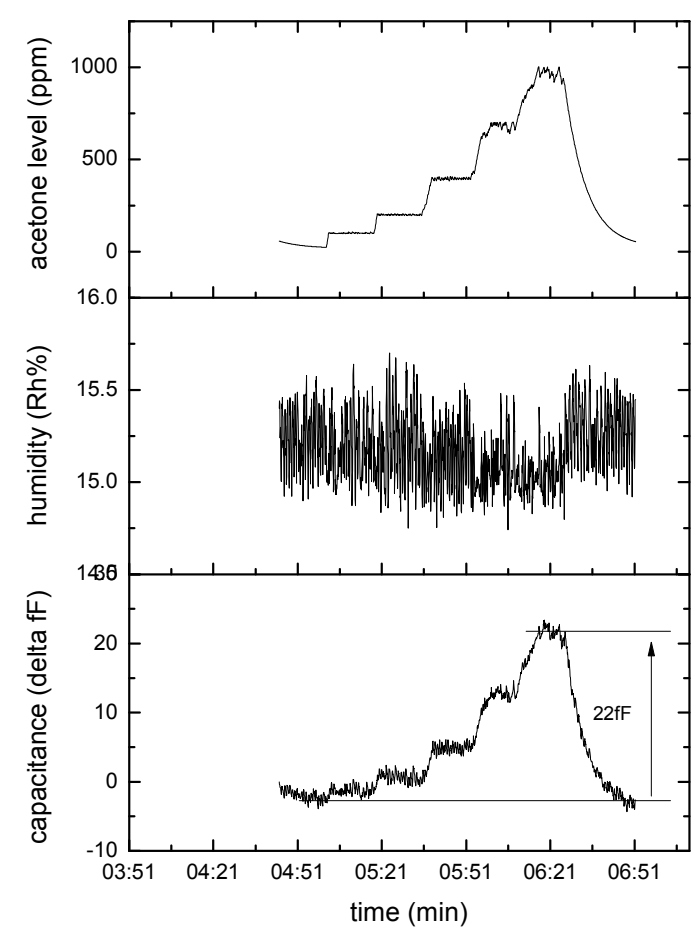

Figure 13. Response of the $8 \mathrm{pF}$ micro-gap IDE with 10\% PVPH polymer to acetone input In order to improve the dynamic range of the measurement, or to improve the output sensor, the same experiment was conducted using a nano-gap IDE. Because of the increased density of the IDE, the capacitance increased to $135 \mathrm{pF}$, including the PVPH sensing layer. The same script pattern used for Figure 13 was executed at the same humidity and temperature. Figure 14 shows the response of the nano-gap IDE. 


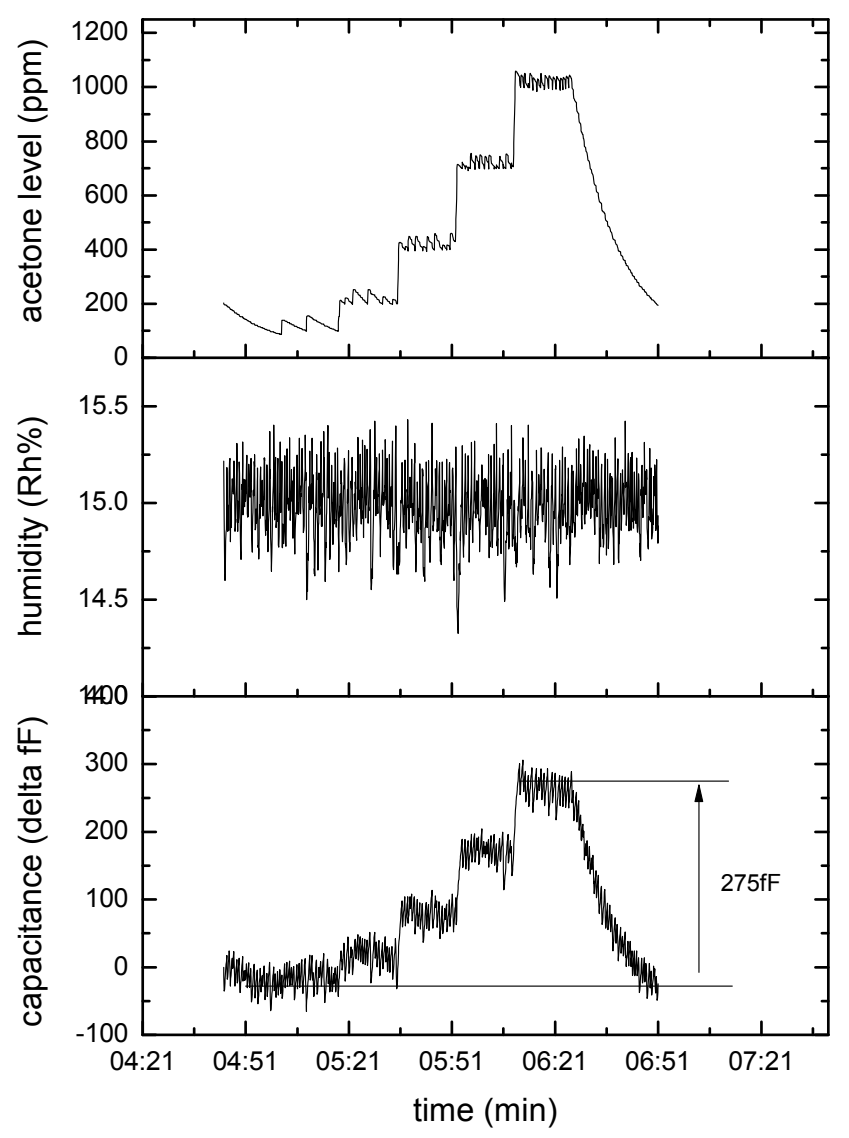

Figure 14. Response of the $135 \mathrm{pF}$ nano-gap IDE with 10\% PVPH polymer to acetone input

Although the response of the sensor to the acetone input and the fluctuation of the humidity is similar to the micro-gap IDE, the signal amplitude increased by a factor of 12 compared to the micro gap sensor, to $275 \mathrm{fF}$ at $1000 \mathrm{ppm}$ acetone, while maintaining the dynamic range of the capacitance interface. Both the micro- and nano-structure showed a $0.2 \%$ change of the capacitance in response to $1000 \mathrm{ppm}$ of acetone. The micro-gap IDE gave a significant response of $22 \mathrm{fF}$ (on $8 \mathrm{pF}$ capacitance) to $1000 \mathrm{ppm}$ acetone while the nano-gap IDE showed a response of $275 \mathrm{fF}$ (on $135 \mathrm{pF}$ capacitance) to 1000ppm acetone. 


\subsection{Discussion}

Recent developments in new semiconductors have made it possible to measure capacitance in the femtofarad range with a single chip semiconductor. The response of the graphene oxide nano-gap sensor is based on the change of the dielectric properties of the sensing layer as it ad/absorbs water vapour as described in various previous studies (Bi et al., 2013; Collignon, Hoang, Picaud, \& Rayez, 2005; Yao, Chen, Guo, Wu, \& $\mathrm{Li}, 2012)$. The non-linear behaviour corresponds with the findings of these groups, although the central frequency of $3.1 \mathrm{MHz}$ used in these experiments higher by a factor of 300 than that reported by other groups (Bi et al., 2013). Previous reported data also shows a bare IDE capacitance between $3 \mathrm{pF}$ and $10 \mathrm{nF}$ with $\mathrm{pF}$ resolution and these measurements were performed with a comparable capacitance, while the limit of detection presented in this paper is smaller by a factor of 1000 , in the femtofarad range, while maintaining reproducibility of the measurement. Furthermore, the limit of detection has improved, enabling the use of comparatively small detection areas. The response time of the GO sample is in the same range as the reference humidity sensor, as shown in Figure 10. Here a change of $100 \mathrm{fF}$ is plotted on the lower graph as a result of a humidity change of $3 \% \mathrm{Rh}$. The response of the sensor is exponential, as Figure 11 shows, and the repeatability is excellent in the lower and higher regions. Calibration of the sensor is possible when fitted to the exponential function. The standard deviation here is calculated at 0.97 .

Furthermore, the increased density of the IDE structure from a micro-gap to a nano-gap results in a larger signal amplitude from the perspective of the increased bare IDE capacitance. This was measured in a second experiment with the same sensing layer properties using Poly (4-vinylphenol) selective for acetone.

The response to temperature changes caused by the resonance circuit components is present in the system, although it is small due to the stability properties of the signal measurement system. If the temperature dependence of a sensor is to be measured, a compensation method is needed. Further improvements of this system may involve a differential measurement approach. In this case a second sensor may be added to the front end that is passivated for the sensing agent but responds to humidity and/or temperature changes at the same level as the active sensor itself. By subtracting both 
signals using digital signal processing, variations caused by temperature or humidity can be minimized (van der Bent et al., 2015).

\subsection{Conclusion}

It is shown that the percentage change of both micro- and nano-gap sensors to an input of $1000 \mathrm{ppm}$ acetone is $0.2 \%$. When the available sensing surface area of the IDE is reduced from micro- to nano-scale, thereby increasing the bare IDE capacitance and thus the absolute value of the $\Delta \mathrm{C}$, a larger part of the available dynamic range of the capacitance measurement device can be used, thus increasing the signal to noise ratio. Next, in order to reduce the change of parasitic capacitance of cabling due to temperature changes and mechanical stress on cables, the measurement should be taken as close to the IDE sensor as possible. The limits of detection in capacitive IDE sensors at room temperature depend heavily on the thermal and mechanical stability of the cabling responsible for parasitic capacitance change and the detection limit or resolution of the measurement device. This work shows that measurements can be conducted in the femtofarad range with high resolution $(0.3 \mathrm{fF})$, while maintaining reproducibility. With this method, the noise floor was measured at $3 \mathrm{fF}$ with $0.3 \mathrm{fF}$ resolution, with a $100 \mathrm{pF}$ value of the IDE including the graphene oxide sensing layer. When the IDE feature size is reduced from the micro- to the nano-scale, the capacitance and corresponding signal amplitude greatly increased. Combining the improved capacitance measurement system and the nano-scale feature sizes of the high-density IDE structures resulted in more than a 10 -old improvement of the limit of detection. This work enables research on highly selective polymers with low dielectric changes. 


\section{Chapter 6}

\section{General discussion}

\subsection{Introduction}

Ideally, sensors should be selective, that is only sensitive to a single parameter. In practice, however, sensors tend to be sensitive to many kinds of interference, also known as cross-sensitivity. If a sensor's selectivity is considered acceptable, it means that the response to the parameter of interest is significantly higher than the response to other factors. However, sensor performance can be hampered even by cross-sensitivity close to the lower detection limit, where the cross-sensitivity easily exceeds the desired response. The detection limit is normally defined as the smallest signal that still can be measured in order to obtain a significant reading, and is often associated with the signalto-noise ratio $(\mathrm{S} / \mathrm{N})$. In addition to the noise floor of the signal, the stability of the sensor over a longer period of time is also a relevant parameter. If the selectivity and detection limit must be optimized, a good environment is necessary to minimize the influence of interference and to maximize the relevant properties of the sensor.

Before any countermeasures can be taken to improve the sensor, all relevant parameters of the sensor in question must be obtained. For example, if a sensor is to be used outdoors, it must be studied with the relevant humidity and temperature ranges. This means that a signal measurement system needs to be designed, constructed and verified that is capable of recreating these conditions in a controlled manner. With such a system, the properties of the experimental prototype sensor being tested can be measured and, with the sensors parameters at hand, countermeasures can be designed and tested. The design of these countermeasures can then be put to the test in the same signal measurement system and before and after data can be analysed and used to improve the sensor system through a process of iteration. The desired outcome of this method is a sensor that can withstand all kinds of environmental variations while still 
producing interpretable data. In the case of a sensor application in a wireless sensor network, data may be reduced in the sensor node itself, thereby reducing the total energy needed to collect, modify and transfer across the ether. From a data perspective this means that the data from the sensor must be interpretable by an algorithm running on a low-power microcontroller or MCU, creating a smart sensor.

\subsection{Limits of Signal Detection and How To Improve it}

This thesis focusses specifically on the improvement of detection limits for different sensor principles. Three separate cases with limiting detection factors are presented and different approach is taken in each case to find the limiting factor and improve the limits of detection. The sensors selected cover a large portion of experimental sensor principles that are currently subject to study, including:

- Resistivity-based nanowire sensors

- Potentiometric sensors generating an electromotive force

- Capacitive sensors with an oscillator operated readout

The introductory Chapter 1 presents an overview of different techniques that can be used and combined to improve the limits of detection. The chapter addresses the current status of signal processing and working towards a smart sensor, including power saving techniques in order to use sensors in an WSN.

It was concluded that an adequate signal measurement system is an important measure for improvement. For each case a custom signal measurement system must be designed in order to obtain relevant parameters from experimental sensors with high precision. The choice is then between a volume controlled environment or a flow controlled environment. The advantage of a volume controlled system over a flow controlled system is that humidity and temperature can both be accurately controlled. In the constructed signal measurement system, the relative humidity can be controlled over a range of 10 to $90 \% \mathrm{Rh}$ with an accuracy of $1 \% \mathrm{Rh}$, and temperature can be controlled over a range of $20^{\circ} \mathrm{C}$ with an accuracy of $0.05^{\circ} \mathrm{C}$. The concentration in the signal measurement system of gasses such as $\mathrm{CO}_{2}, \mathrm{H}_{2}$ and/or $\mathrm{N}_{2}$ can be controlled well by a computer script. The script is composed of a number of program lines indicating the desired values in the signal measurement system. This script is executed by a control PC 
running on a high-level graphical language that controls and reads all valves, pumps, control electronics and calibrated reference sensors in the system. A script file can be run once or any number of times in order to improve the significance of measurement values. All readout values and set points are collected by the control PC and written to an Excel file for further analysis.

Equipped with these resources, an initial attempt was made in Chapter 3 to improve the limits of detection for a resistivity based nanowire sensor able to detect hydrogen. Here the sensor itself consists of a mirrored array of two palladium nanowires. When the palladium nanowires are exposed to hydrogen, they absorb the gas and the wires expand. The swelling of the nanowires in turn produces a $\Delta \mathrm{R}$ change in the resistance. Although the $\Delta \mathrm{R}$ change is a fraction of the bulk value $\left(0.96 \sigma / \sigma_{\mathrm{o}}\right.$ at $\left.12,000 \mathrm{ppm} \mathrm{H}_{2}\right)$, the limit of detection proved to be determined by the cross-sensitivity of the sensor to temperature. This effect was analysed using a volume controlled signal measurement system. Because the temperature in the measurement system is known, an initial compensation method is presented in which the chamber temperature is used for compensation, lowering the limit of detection. Although this method is promising, it fails when the system is to be used for an early warning rapid response system because of the inequality in the time constants $(\tau)$ of the temperature sensor and the nanowires. $A$ near perfect compensation would only be possible if the temperature constants matched, and this is demonstrated by using two nanowire sensors. One of the nanowires is open to ad/absorb hydrogen while the other is passivated for hydrogen but not for temperature changes. These signals were both digitized and fed to a digital signal processing algorithm that both subtracted the signal and lowered the effect of hydrogen penetrating the passivation layer. The outcome of this experiment was a high-speed (500-600ms) response to relatively small changes in the amount of hydrogen (2000ppm), enabling the system to be used in a wireless sensor network for early detection purposes and for measuring the absolute concentration, after calibration, within 15 seconds.

The second example (Chapter 4) attempted to improve the limits of detection in potentiometric sensors with a focus on the detection of carbon dioxide. For this experiment, a commercially available sensor was used with a custom designed lowpower amplifier and analogue-to-digital converter that could facilitate four differential 
sensor readouts at the same time. A volume controlled signal measurement system was used to characterize the sensors. Since the temperature is part of the Nernst equation, this parameter plays an important role in the output voltage of the sensor. Although the Nernst equation does not include time, it was shown that time plays a significant role in the baseline stability of the sensor. It is shown that this baseline drift is somewhat erratic and varies from sensor to sensor. When two sensors are matched, both sensors can be used simultaneously to reduce the baseline drift as a result of temperature variations. When one of the sensors is passivated while the other remains open to carbon dioxide, they show the same response but differ in amplitude and baseline voltage. With an algorithm that matches and combines the output of the sensors, a $4000 \mathrm{ppm}$ input of $\mathrm{CO}_{2}$ was measured over a temperature variation of $22^{\circ} \mathrm{C}$.

Because measurements could be repeated over time using scripts, the long-term properties in depletion of the sensor were also studied, providing insight into the wear of the sensor over time. This resulted in a depletion parameter that can be used to correct the output of the sensor, making it more accurate over a long period of time.

A third example (Chapter 5) again investigates lowering the limits of detection, this time using a capacitive measuring system to cover another wide range of sensors. First the measurement of the capacitance itself is analysed in detail, and three possible aspects within the measurement were then isolated that could be used to improve the detection limit. First, the two-dimensional interdigitated electrode (IDE) was examined. The bulk value of this capacitor is determined by the scale and size of the surface structure and the sensing layer serving as a dielectricum and by changing the value of the bulk by a $\Delta$ factor as the dielectricum swells or shrinks after selectively ab/adsorbing vapour. Downscaling to nano-scale has the advantage of increasing the $\Delta$ change in capacitance caused by ab/adsorption. This means that if a change of $5 \%$ is to be expected and the bulk value of the capacitor is $1 \mathrm{pF}$, the total $\Delta$ change will be $5 \mathrm{fF}$. When a structure is reduced to a nano-scale of $440 \mathrm{~nm}$, the bulk capacitance increases to $50 \mathrm{pF}$, thus increasing the $\Delta$ change to $50 \mathrm{fF}$. This reasoning led to the development of a capacitor with a bulk value that is fairly high and a structure that is fairly small. After connecting the IDE to the measuring device, the test was begun. It was found that, due to temperature fluctuation and possible cable artefacts, additional capacitive noise was added to the signal during the measurement. It was also found that a standard RG58 
cable between the sensing capacitor and the measuring device added $31.25 \mathrm{fF} /{ }^{\circ} \mathrm{C} / \mathrm{m}$; the cable therefore influenced the measuring range. In order to reduce this effect as much as possible, the cable length should be reduced as much as possible (approaching zero). This led to the design of a capacitance interface near the sensor with a stable parasitic capacitance. A capacitance interface was designed, constructed and verified with a $0.3 \mathrm{fF}$ resolution and capable of handling bulk capacitance values up to $250 \mathrm{nF}$. A combination of the nano-scale IDE with a graphene oxide sensing layer and the capacitance interface led to measurement results that were comparable with other work performed in the picofarad range, with the exception that these measurements were taken in a femtofarad range while maintaining high resolution. The experiment with acetone shows that a reduction of the surface IDE structure to a nano-scale combined with taking measurements in close proximity to the experimental chip produces the most promising results. When the bulk value of the capacitor increases due to nano-scaling while the sensing area remains the same, the $\Delta$ change also increases due the absorption of acetone in the sensing layer. The difference in response, or signal amplitude, between micro- (8 pf bulk $\mathrm{C}$ ) and nano-scaling (135 pF bulk $\mathrm{C}$ ) is demonstrated. Here, the microand nano-IDE chips were prepared with an equal layer of PVPH 10\% and were subjected to the same environment and the same acetone levels. The enlarged $\Delta \mathrm{C}$ signal of the IDE because of the downscaling of the IDE structure to nano-structures makes it possible to experiment with selective polymers when combined with a high-resolution capacitance measurement.

Both the micro- and nano-structure showed a $0.2 \%$ change in the bulk capacitance at $1000 \mathrm{ppm}$ of acetone. The micro-gap IDE gave a significant response of $22 \mathrm{fF}$ to $1000 \mathrm{ppm}$ acetone while the nano-gap IDE produced a response of $275 \mathrm{fF}$ to $1000 \mathrm{ppm}$ acetone.

\subsection{Conclusions}

In general, it can be concluded that the importance of a well-defined signal measurement system and the time required to find the optimal measuring solution mean that these aspects require a large portion of the total research time available. Although the signal measurement system described in Chapter 2 could be further improved in future research, it is, in its present state, capable of interfacing with a large spectrum of sensor principles, each involving minor changes in the structure of the 
signal measurement system. Improvements of the system could be made by controlling the input flow into the chamber. If this flow were to be set more accurately, the response time of the chamber could be improved when setting the flow to a higher value, combined with a low flow when the set point is nearly reached to improve the accuracy of the chamber. Furthermore, in this study a Peltier element of $100 \mathrm{~W}$ was used to control the temperature in the chamber. It would be advisable to increase the power of this element in order to operate in a larger range and to decrease the time needed to reach the desired set point.

A temperature balanced, fast and selective early hydrogen detection method is presented in Chapter 3. Two identical palladium nanowires were used in order to match time constants. The work showed that although the temperature had a large influence on the measurement signal, the absolute temperature need not be measured. It was found that when sensors are constructed and the measurement method is clear, two identical sensors can be used simultaneously to suppress unwanted cross-sensitivity. This is done in a more precise way than with absolute temperature compensation, because the time constants of the identical palladium sensors matched perfectly. Chapter 4 demonstrated that matching potentiometric based sensors that produce a small voltage can be used to predict the drift of the baseline signal. It was shown that this method worked in a broad temperature range. Furthermore, it was found that when a proper signal measurement system is present, the relevant data can be collected regarding the depletion process of the sensor, leading to a more accurate readout value. Future work could involve capturing the influence of temperature fluctuations in order to predict the amount and the direction of the baseline drift.

Downscaling the interdigitated electrode substrate structure to a nano-level, combined with a direct measurement and using state-of-the-art capacitance measuring led to high resolution $(0.3 \mathrm{fF})$ measurements in the femtofarad range (Chapter 5$)$. This technique enables researchers to work with thin highly selective polymer layers while maintaining a high resolution readout. Future work may include combining the techniques described in chapters 3 and 4, using a reference capacitor in order to decrease cross-sensitivity due to temperature variations. For this, a new single silicon chip must be developed with multiple IDE structures with updated differential readout front-end electronics. 


\section{References}

\section{References}

Abdullah, Q. N., Yam, F. K., Hassan, J. J., Chin, C. W., Hassan, Z., \& Bououdina, M. (2013). High performance room temperature GaN-nanowires hydrogen gas sensor fabricated by chemical vapor deposition (CVD) technique. International Journal of Hydrogen Energy, 38(32), 14085-14101. doi: 10.1016/j.ijhydene.2013.08.014

Abdullah, Q. N., Yam, F. K., Hassan, Z., \& Bououdina, M. (2014). Hydrogen gas sensing performance of GaN nanowires-based sensor at low operating temperature. Sensors and Actuators B: Chemical, 204(0), 497-506. doi: http://dx.doi.org/10.1016/j.snb.2014.07.112

Alley, G. D. (1970). Interdigital Capacitors and Their Application to Lumped-Element Microwave Integrated Circuits. IEEE Transactions on Microwave Theory and Techniques, 18(12), 1028-1033. doi: 10.1109/TMTT.1970.1127407

Atashbar, M. Z., Banerji, D., \& Singamaneni, S. (2005). Room-temperature hydrogen sensor based on palladium nanowires. Sensors Journal, IEEE, 5(5), 792-797.

Bakker, Eric, \& Pretsch, Ernö. (2005). Potentiometric sensors for trace-level analysis. TrAC Trends in Analytical Chemistry, 24(3), 199-207. doi: http://dx.doi.org/10.1016/j.trac.2005.01.003

Barreca, Davide, Bekermann, Daniela, Comini, Elisabetta, Devi, Anjana, Fischer, Roland A., Gasparotto, Alberto, ... Tondello, Eugenio. (2010). 1D ZnO nano-assemblies by Plasma-CVD as chemical sensors for flammable and toxic gases. Sensors and Actuators B: Chemical, 149(1), 1-7. doi: http://dx.doi.org/10.1016/j.snb.2010.06.048

Bi, Hengchang, Yin, Kuibo, Xie, Xiao, Ji, Jing, Wan, Shu, Sun, Litao, ... Dresselhaus, Mildred S. (2013). Ultrahigh humidity sensitivity of graphene oxide. Scientific Reports, 3 , 2714. doi: 10.1038/srep02714 http://www.nature.com/articles/srep02714\#supplementary-information 
CHEMSAFE. (2004). A database of evaluated safety characteristics. Frankfurt/M.: CHEMSAFE.

Cheng, Yang-Tse, Li, Yang, Lisi, Dan, \& Wang, W. M. (1996). Preparation and characterization of $\mathrm{Pd} / \mathrm{Ni}$ thin films for hydrogen sensing. Sensors and Actuators B: Chemical, 30(1), 11-16.

Collignon, B, Hoang, PNM, Picaud, S, \& Rayez, JC. (2005). Ab initio study of the water adsorption on hydroxylated graphite surfaces. Chemical physics letters, 406(4), 430-435.

Doan, Tin C. D., Ramaneti, Rajesh, Baggerman, Jacob, van der Bent, J. Franc, Marcelis, Antonius T. M., Tong, Hien D., \& van Rijn, Cees J. M. (2012). Carbon dioxide sensing with sulfonated polyaniline. Sensors and Actuators B: Chemical, 168(0), 123-130. doi: http://dx.doi.org/10.1016/j.snb.2012.03.065

Evgeny, Fomin. (2013). LDC1000 Temperature Compensation. SNAA212, 7. http://www.ti.com/lit/an/snaa212/snaa212.pdf

Fadel, Etimad, Gungor, V. C., Nassef, Laila, Akkari, Nadine, Malik, M. G. Abbas, Almasri, Suleiman, \& Akyildiz, Ian F. (2015). A survey on wireless sensor networks for smart grid. Computer Communications, 71, 22-33. doi: http://dx.doi.org/10.1016/j.comcom.2015.09.006

Farahani, Hamid, Wagiran, Rahman, \& Hamidon, Mohd. (2014). Humidity Sensors Principle, Mechanism, and Fabrication Technologies: A Comprehensive Review. Sensors, 14(5), 7881.

Favier, Frédéric, Walter, Erich C., Zach, Michael P., Benter, Thorsten, \& Penner, Reginald M. (2001). Hydrogen Sensors and Switches from Electrodeposited Palladium Mesowire Arrays. Science, 293(5538), 2227-2231. doi: 10.1126/science.1063189

Figaro USA, INC. (2005). TGS 4161 - for the detection of Carbon Dioxide. https://www.sos.sk/productdata/53/10/3/53103/tgs4161.pdf

Fleischer, M., Ostrick, B., Pohle, R., Simon, E., Meixner, H., Bilger, C., \& Daeche, F. (2001). Low-power gas sensors based on work-function measurement in low-cost hybrid flip-chip technology. Sensors and Actuators B: Chemical, 80(3), 169-173.

Hagleitner, C., Hierlemann, A., Lange, D., Kummer, A., Kerness, N., Brand, O., \& Baltes, H. (2001). Smart single-chip gas sensor microsystem. Nature, 414(6861), 293-296. Harbeck, S., Szatvanyi, A., Barsan, N., Weimar, U., \& Hoffmann, V. (2003). DRIFT studies of thick film un-doped and Pd-doped SnO2 sensors: temperature changes effect 
and $\mathrm{CO}$ detection mechanism in the presence of water vapour. Thin Solid Films, 436(1), 76-83.

Harraz, Farid A., Ismail, Adel A., Bouzid, Houcine, Al-Sayari, S. A., Al-Hajry, A., \& Al-Assiri, M. S. (2014). A capacitive chemical sensor based on porous silicon for detection of polar and non-polar organic solvents. Applied Surface Science, 307, 704-711. doi: http://dx.doi.org/10.1016/j.apsusc.2014.04.106

Hobdell, J. L. (1979). Optimization of Interdigital Capacitors. IEEE Transactions on Microwave Theory and Techniques, 27(9), 788-791. doi: 10.1109/TMTT.1979.1129730

Horowitz, P, \& Hill, W. (2015). The art of electronics. [England]: Cambridge University Press.

Huang, Bohr-Ran, \& Lin, Jun-Cheng. (2012). Core-shell structure of zinc oxide/indium oxide nanorod based hydrogen sensors. Sensors and Actuators B: Chemical, 174(0), 389-393. doi: http://dx.doi.org/10.1016/j.snb.2012.08.065

Igreja, Rui, \& Dias, C. J. (2004). Analytical evaluation of the interdigital electrodes capacitance for a multi-layered structure. Sensors and Actuators A: Physical, 112(2-3), 291-301. doi: http://dx.doi.org/10.1016/j.sna.2004.01.040

Im, Yeonho, Lee, Choonsup, Vasquez, Richard P, Bangar, Mangesh A, Myung, Nosang V, Menke, Eric J, ... Yun, Minhee. (2006). Investigation of a Single Pd Nanowire for Use as a Hydrogen Sensor. Small, 2(3), 356-358. doi: 10.1002/smll.200500365

Joshi, Rakesh, Krishnan, Subramanian, Yoshimura, Mashamichi, \& Kumar, Ashok. (2009). Pd Nanoparticles and Thin Films for Room Temperature Hydrogen Sensor. Nanoscale Research Letters, 4(10), 1191-1196.

Kahn. (july 2012). Use of Optical Hygrometers as Reference Instruments in Calibration Laboratories Retrieved March 04, 2016, from http://www.kahn.com/hygrometers/ReferenceInstruments.pdf

Kim, Kyung S., \& Paik, Woon-Kie. (1975). Effects of temperature and pressure on conductance of solid electrolyte, rubidium silver iodide. Journal of Chemical \& Engineering Data, 20(4), 356-359. doi: 10.1021/je60067a021

Kim, Youngdeuk, Jung, Bongbu, Lee, Hunkee, Kim, Hyejin, Lee, Kunhong, \& Park, Hyunchul. (2009). Capacitive humidity sensor design based on anodic aluminum oxide. Sensors and Actuators B: Chemical, 141(2), 441-446. doi: http://dx.doi.org/10.1016/j.snb.2009.07.007 
Kyun Tae, Kim, Jun, Sim, \& Sung Min, Cho. (2006). Hydrogen gas sensor using Pd nanowires electro-deposited into anodized alumina template. Sensors Journal, IEEE, 6(3), 509-513.

Marsal, A., Centeno, M. A., Odriozola, J. A., Cornet, A., \& Morante, J. R. (2005). DRIFTS analysis of the $\mathrm{CO} 2$ detection mechanisms using $\mathrm{LaOCl}$ sensing material. Sensors and Actuators B: Chemical, 108(1-2), 484-489.

Maruyama, Toshio, Sasaki, Seihiro, \& Saito, Yasutoshi. (1987). Potentiometric gas sensor for carbon dioxide using solid electrolytes. Solid State Ionics, 23(1-2), 107-112.

Metting van Rijn, A., Peper, A., \& Grimbergen, C. (1990). High-quality recording of bioelectric events. Medical and Biological Engineering and Computing, 28(5), 389-397. doi: 10.1007/bf02441961

Mladen, Berekovic. (2007). Ulta-Low-Power Wireless Sensor Node Design on 100 uW Scavenging Energy for Applications In Biomedical Monitoring.

Obata, Kenji, Kumazawa, Shizuko, Matsushima, Shigenori, Shimanoe, Kengo, \& Yamazoe, Noboru. (2005). NASICON-based potentiometric CO2 sensor combined with new materials operative at room temperature. Sensors and Actuators B: Chemical, 108(1-2), 352-358.

Offermans, P., Tong, H. D., Rijn, C. J. M. van, Merken, P., Brongersma, S. H., \& CregoCalama, M. (2009). Ultralow-power hydrogen sensing with single palladium nanowires. Applied Physics Letters, 94(22), 223110.

Roth, M., Hartinger, R., Faul, R., \& Endres, H. E. (1996). Drift reduction of organic coated gas-sensors by temperature modulation. Sensors and Actuators B: Chemical, 36(1-3), 358-362.

Satyanarayana, L., Noh, Whyo Sup, Lee, Woon Young, Jin, Gwang Hu, \& Park, Jin Seong. (2009). A high temperature potentiometric CO2 sensor mixed with binary carbonate and glassy ceramic oxide. Materials Chemistry and Physics, 114(2-3), 827-831. doi: http://dx.doi.org/10.1016/j.matchemphys.2008.10.065

Scharfetter, H., Hartinger, P., Hinghofer-Szalkay, H., \& Hutten, H. (1998). A model of artefacts produced by stray capacitance during whole body or segmental bioimpedance spectroscopy. Physiological Measurement, 19(2), 247.

Sensors_online. (2001). Choosing a Humidity Sensor: A Review of Three Technologies. Retrieved March 01, 2016, from 
http://www.sensorsmag.com/sensors/humidity-moisture/choosing-a-humiditysensor-a-review-three-technologies-840

Shrivastava, Alankar, \& Gupta, Vipin. (2011). Methods for the determination of limit of detection and limit of quantitation of the analytical methods. Chronicles of Young Scientists, 2(1), 21-25. doi: 10.4103/2229-5186.79345

Smith, Steven W. (1997). The scientist and engineer's guide to digital signal processing: California Technical Publishing

Texas_instruments. (2015). FDC2x1x EMI-Resistant 28-Bit,12-Bit Capacitance-to-Digital Converter for Proximity and Level Sensing Applications. 60. http://www.ti.com/lit/ds/symlink/fdc2114.pdf

Tong, Duy Hien, Chen, Songyue, Wiel van der, Wilfred G., Carlen, Edwin T., \& Berg van den, Albert. (2009). Novel Top-Down Wafer-Scale Fabrication of Single Crystal Silicon Nanowires. Nano Letters, 9(3), 1015-1022.

van der Bent, J. F., Puik, E., Tong, H. D., \& van Rijn, C. J. M. (2015). Temperature balanced hydrogen sensor system with coupled palladium nanowires. Sensors and Actuators A: Physical, 226(0), 98-106. doi: http://dx.doi.org/10.1016/j.sna.2015.01.037

van der Bent, J. F., van Rijn, C. J. M., \& Puik, E. (2010). Ultra low power temperature compensation method for palladium nanowire grid. Procedia Engineering, 5, 184187.

Wang, Changhong, Lu, Wei, Narayanan, Michael R., Redmond, Stephen J., \& Lovell, Nigel H. (2015). Low-power technologies for wearable telecare and telehealth systems: A review. Biomedical Engineering Letters, 5(1), 1-9. doi: 10.1007/s13534-0150174-2

Wang, Jun, Jiu, Jinting, Nogi, Masaya, Sugahara, Tohru, Nagao, Shijo, Koga, Hirotaka, .. . Suganuma, Katsuaki. (2015). A highly sensitive and flexible pressure sensor with electrodes and elastomeric interlayer containing silver nanowires. Nanoscale, 7(7), 2926-2932. doi: 10.1039/C4NR06494A

Wolfe, Daniel B., Love, J. Christopher, Paul, Kateri E., Chabinyc, Michael L., \& Whitesides, George M. (2002). Fabrication of palladium-based microelectronic devices by microcontact printing. Applied Physics Letters, 80(12), 2222-2224.

Yamazoe, N., Hisamoto, J., Miura, N., \& Kuwata, S. (1987). Potentiometric solid-state oxygen sensor using lanthanum fluoride operative at room temperature. Sensors 
and Actuators, 12(4), 415-423. doi: http://dx.doi.org/10.1016/0250-

6874(87)80060-4

Yang, San Xu, \& Yang, Wu Qiang. (2002). A portable stray-immune capacitance meter. Review of Scientific Instruments, 73(4), 1958-1961. doi: doi:http://dx.doi.org/10.1063/1.1453504

Yao, Yao, Chen, Xiangdong, Guo, Huihui, Wu, Zuquan, \& Li, Xiaoyu. (2012). Humidity sensing behaviors of graphene oxide-silicon bi-layer flexible structure. Sensors and Actuators B: Chemical, 161(1), 1053-1058. doi:

http://dx.doi.org/10.1016/j.snb.2011.12.007

Zaal, T. (2009). Intergrated Design and Engineering - as a Business Improvement Process. Utrecht: Maj Engineering Publishing.

Zaretsky, M. C., Melcher, J. R., \& Cooke, C. M. (1988, 5-8 June 1988). Moisture sensing in transformer oil using thin film microdielectrometry. Paper presented at the Electrical Insulation, 1988., Conference Record of the 1988 IEEE International Symposium on.

Zhou, R., Hierlemann, A., Weimar, U., \& Göpel, W. (1996). Gravimetric, dielectric and calorimetric methods for the detection of organic solvent vapours using poly(ether urethane) coatings. Sensors and Actuators B: Chemical, 34(1), 356360. doi: http://dx.doi.org/10.1016/S0925-4005(96)01912-0 


\section{Appendix A}

\section{Capacitance Interface Design ${ }^{4}$}

\section{A1. Introduction}

The measurements in Chapter 5 of acetone concentrations and, separately, humidity, were taken with a custom-built capacitance measurement interface. This appendix presents the background information for the design and verification of the interface itself, since the device is experimental and the guidance in existing literature is very limited. It should be noted that all verifications were performed with calibrated measuring equipment and that all ESD guidelines were followed. All measurement values collected were in line with the expected values, or better.

\section{A1.1. Selecting the Silicon Chip}

Capacitance can be measured in numerous ways with a wide variety of systems. Some digital multimeters (DMMs) can perform a capacitance measurement but have a limited resolution in the $1 \mathrm{pF}$ range. In this system the goal is on the order of $10 \mathrm{fF}$ resolution, roughly 100 times higher. Recent developments in semiconductors with respect to capacitance sensing has produced a range of chips that can be used for level control. These chips have a digital interface for the readout and can measure in the range of femtofarads and below. Internet research shows that there are two large semiconductor companies producing devices that can measure in this range. Table 1 shows the chips currently available (Q4, 2015).

Table 1, Selection table for cap sense devices

\begin{tabular}{|l|l|r|l|l|l|l|l|}
\hline company & type & resolution bits & setp resolution & common mode cap & diff mode & single ended & remark \\
\hline analog devices & AD7152 & 12 & $0.25 \mathrm{fF}$ & $<5 \mathrm{pF}$ & $0.25 \mathrm{pF}$ to $2 \mathrm{pF}$ & $0.5 \mathrm{pF}$ to $4 \mathrm{pF}$ & $1 \mathrm{channel}$ \\
\hline analog devices & AD7747 & 24 & $20 \mathrm{aF}$ & $<8 \mathrm{pF}$ & $8 \mathrm{pF}$ & $17 \mathrm{pF}$ & $1 \mathrm{channel}$ \\
\hline analog devices & AD7142 & 16 & $1 \mathrm{fF}$ & & & $20 \mathrm{pF}$ & slider interface \\
\hline analog devices & AD7745 & 24 & $4 \mathrm{aF}$ & $17 \mathrm{pF}$ & $4 \mathrm{pF}$ & $1 \mathrm{channel}$ \\
\hline freescale Semi & MPR121 & 8 & $?$ & $>?$ & $2 \mathrm{pF}$ & $100 \mathrm{pF}$ stray & 12 channel not calibrated \\
\hline texas instruments & FDC2212 & 28 & $0.3 \mathrm{fF}$ & $250 \mathrm{nF}$ & $\mathrm{nF}$ & 2 channels \\
\hline
\end{tabular}

\footnotetext{
${ }^{4}$ Adapted from the report "Test Chamber Setup for an Experimental Interdigitated Electrode Micro Gap
} Based Sensor", 2016, J.F. van der Bent 
Although resolution is an excellent criterion here and helps narrow down and select the most suitable device accordingly, the common mode capacitor value indicates the maximum bare IDE capacitance that can be handled. Our reference IDE has a bare IDE capacity between 30 and $50 \mathrm{pF}$, at time of selection, guiding the selection away from choosing analogue devices device towards the Texas Instruments chip that can handle $250 \mathrm{nF}$ bulk capacitance. The FDC2x1x proximity and level sensing series is available in several types, differing in resolution and number of available channels

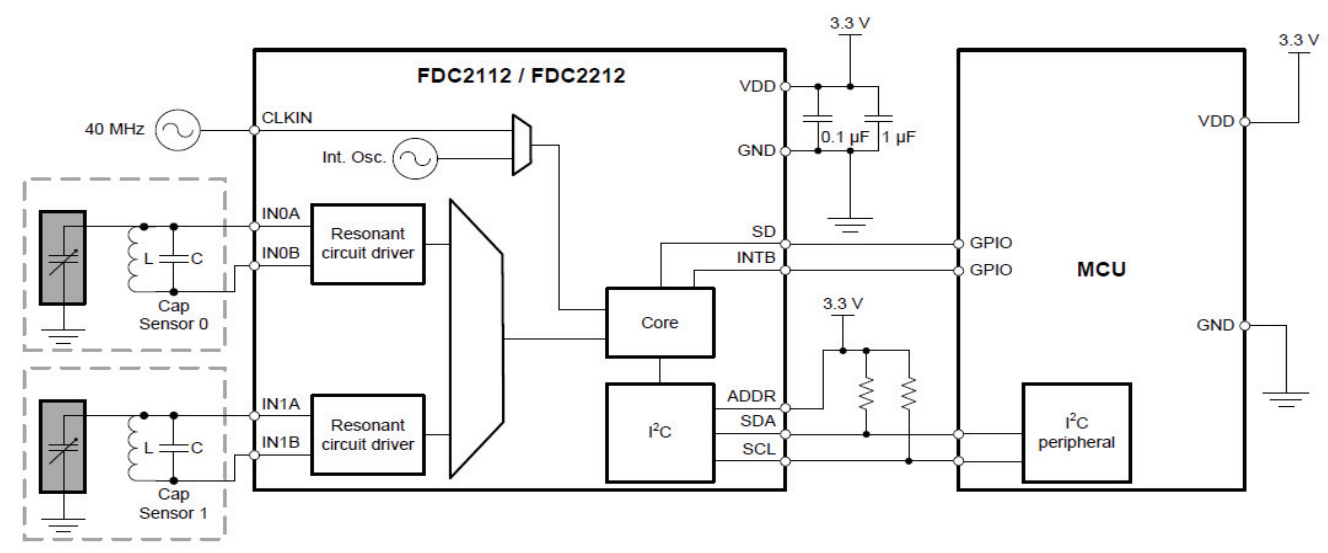

Figure 1. Block diagram of the FDC2212 (source FDC2x2x datasheet)

(Texas_instruments, 2015). The FDC2212 is a chip in a WSON package with two-channel cap sensing with a $0.3 \mathrm{fF}$ resolution $168 \mathrm{~dB}$ dynamic range, equipped with the industry standard I²C (Inter IC Communication) MCU interface (see Figure 1).

The working principle of the sensor is to bring the input, with the help of a programmable oscillator, to its resonant frequency with an added static coil (L) and capacitor (C). A deviation from the internal oscillator can be measured after a multiplexer with a 28 bit, $168 \mathrm{~dB}$ dynamic range converter core. The resonant driver circuit autonomously feeds the core with the $\mathrm{F}_{0}$ frequency of the tank circuit. Since the micro-gap sensor itself is part of the tank circuit, the deviation in frequency is measured by comparison with a reference oscillator. The MCU hosting the $\mathrm{I}^{2} \mathrm{C}$ peripheral can be a generic type. 


\section{A2. Designing the Interface}

The MCU controller board

The chip interface, as introduced above, must be connected to the base controller board. This controller PCB, or ARMboard, was designed for educational purposes by the author on the basis of the NXP LPC2148 ARM processor.

\section{A2.2. Interfacing the FDC2212}

The ARMboard is capable of hosting the $\mathrm{I}^{2} \mathrm{C}$ connection as a bus master for interfacing with the FDC2212, but software needs to be developed for the task of configuring the chip and reading the data registers in order to obtain an output value. The $\mathrm{I}^{2} \mathrm{C}$ is an industry standard protocol developed by Philips and dating back to the early 1980s. The interface consists of two wires, clock and data. The $\mathrm{I}^{2} \mathrm{C}$ is a multi-chip interface and can accommodate up to 127 different devices in one chain as long as the line capacitance is kept under $400 \mathrm{pF}$. The interface is a half-duplex and every chip in the chain must have a unique address. There is one bus-master, in this case the LPC2148 MCU, although the protocol supports multi-master addressing. In this chain only one device is inserted, namely the FDC. Expansion port SV1 of the ARMboard holds the $\mathrm{I}^{2} \mathrm{C}$ interface at pins P3 (SDa, Serial Data) and P2 (SCl Serial Clock). The maximum data rate of the chip is 400 $\mathrm{kHz}$ but it also supports static data transfer, meaning that lower data rates are acceptable. In addition to the data connections and power connections, the chip is equipped with an interrupt pin, an output and a shutdown (SD) pin. The shutdown is not used in this design, as this functionality is commonly used in battery powered mobile equipment where power usage is relevant. The INTB pin may be used to inform the controller of a change in the system status. This pin is connected to one of the external interrupt pins of the ARMboard and reserved for future use. Since only one FDC2212 is used, the ADDR pin has a static connection with either ground or VCC. The chip is equipped with two channels for conversion of capacitance, although only one channel is used in the design. 


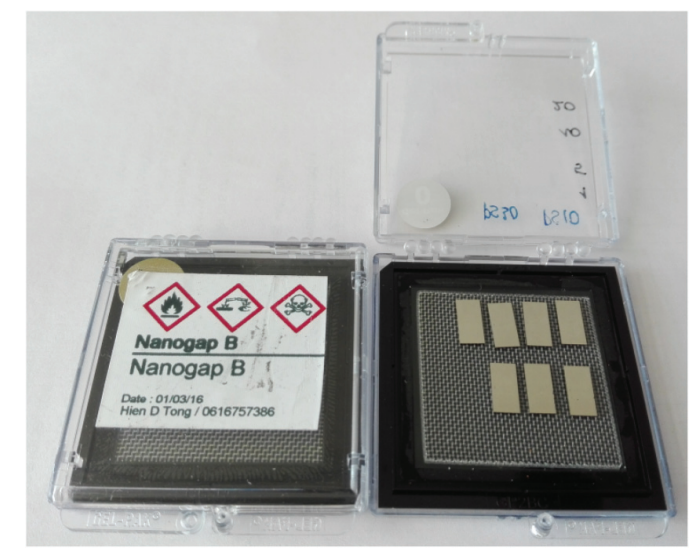

Figure 2. Experimental nano-gap chips $5 \times 10 \mathrm{~mm}$

The target device under test (DUT) or sensor chip has two terminals and should be easily replaceable by another while testing the functionality. The contacts should be firm enough to ensure low contact resistance $<0.1 \Omega$ in order to keep instability of the contacts as low as possible while maintaining a short changeover time. Normally, experimental chips are shipped in a small container, as seen in Figure 2, and are removed from the sticky conduction layer of the box with plastic tweezers for further handling.

The contact pads of the current chip versions are $3.4 \mathrm{~mm}$ by $4 \mathrm{~mm}$ but are subject to change. The contact surface of the interdigitated finger and sensing material, e.g. selective polymer, has the largest change in capacitance when the capacitance is as high as possible, which may lead to small contact pads in need of contact point precision.

\section{A2.3. Designing the Capacitance Interface}

The schematic was designed using Eagle schematic and PCB design suite. Information about the pins and packages was provided by the datasheet, as was information about decoupling. Since only one input of the FDC2212 was used, the other input was given SMD test pads for debug usage during development. The external clock input was also designed with an SMA (sub-miniature version A) connector, although the FDC2212 also has an internal reference oscillator. Information about capacitor values was adapted from the datasheet of Texas Instruments, leading to the schematic design in figure 3. 


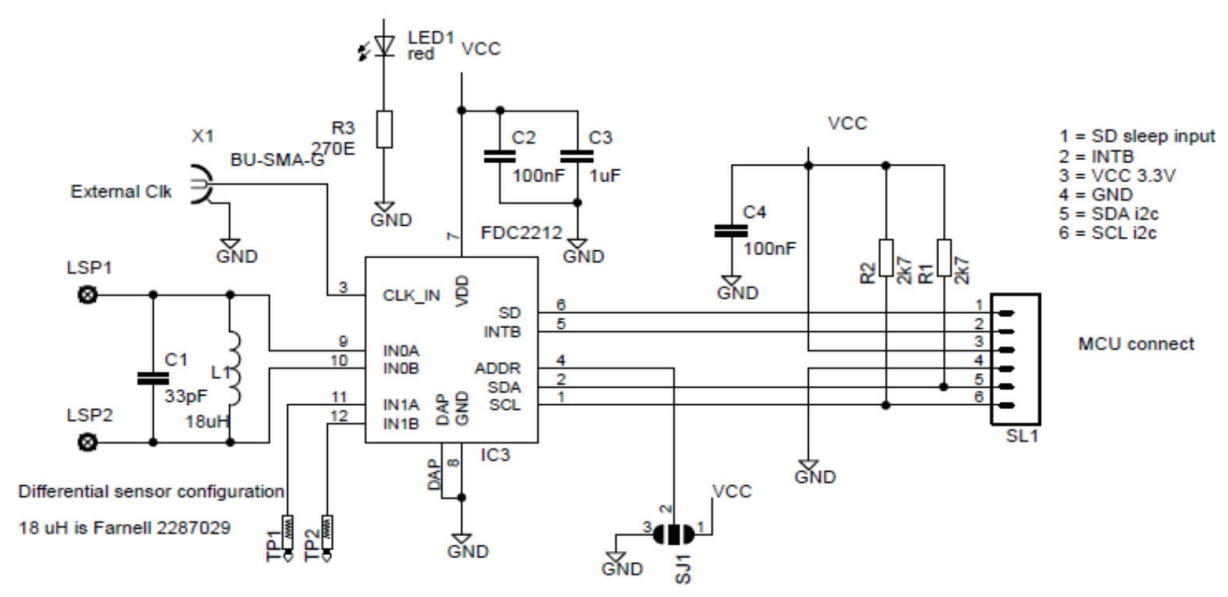

Figure 3. Schematic of the FDC2212 chip with interfacing to the micro-gap chip connected to the LSP1 and LSP 2 connections

The double-sided printed circuit board was designed using the Texas Instruments reference design. This means, among other design rules, that the polygon layer of the ground does not extend towards the sensing boundary of the design. Furthermore, the PCB has two parts separated by milling for easy separation. Input A was used and leads are symmetrically placed towards the pogo pins and the reference inductor and reference capacitor. The final value of the reference capacitor depends on the measured bare IDE capacity of the micro-gap sensor and needs to be adjusted accordingly.

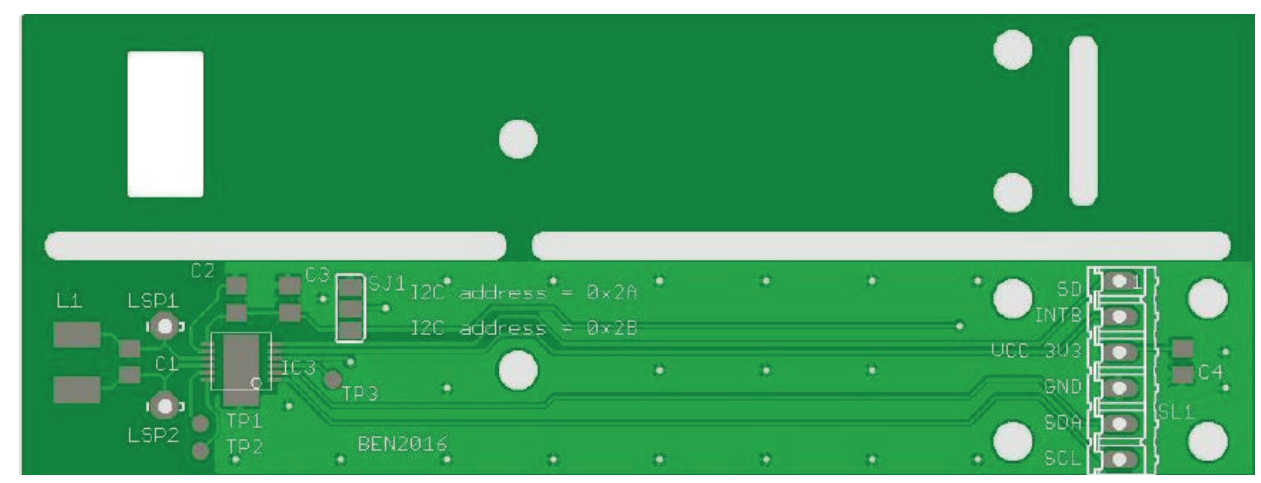

Figure 4. The V1.0 design of the PCB after analysis of the files by the PCB manufacturer 


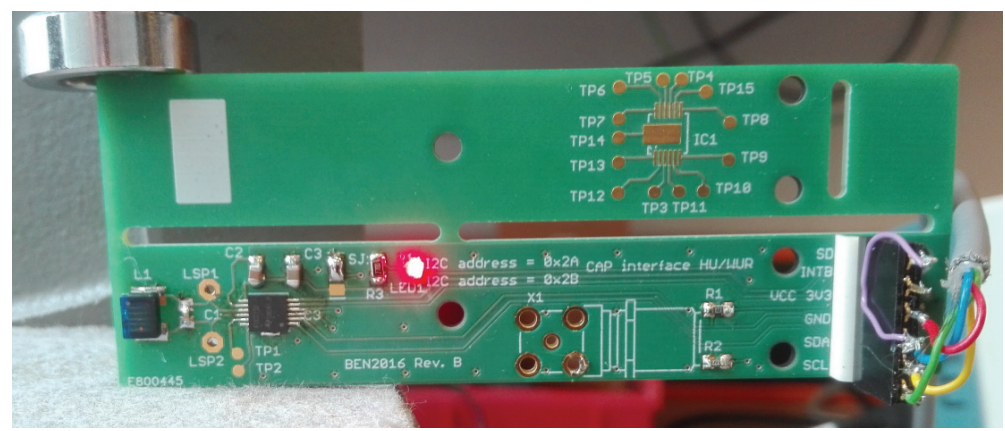

Figure 5. Assembled V2.1 PCB in test setup

Figure 5 shows the assembled PCB in uncut form running test software. Connector X1 can be used to accommodate an SMA connector, enabling the setup to run from an external clock as opposed to the use of the internal oscillator. The top section of this figure reveals the optional second test layout for interfacing the FDC. This circuit is optional and only of use during debugging and verification of the PCB floor plan.

\section{A2.4. Chip Holder Design}

The chip holder, placed at the white square in the top section of the PCB, was designed with Solid Works and 3D printed (see Figure 6). The chip holder is a squared box with space left out, referred to as the sensor bed, for the micro-gap chip. The top edges of the box have a filet of $1 \mathrm{~mm}$ and the bottom filet is $0.5 \mathrm{~mm}$ for easy access to the chip. The cylindrical extrusions were used to ensure that no material was deposited in the corners. PLA (PolyLActide) material was used with no fill degree to ensure flatness of

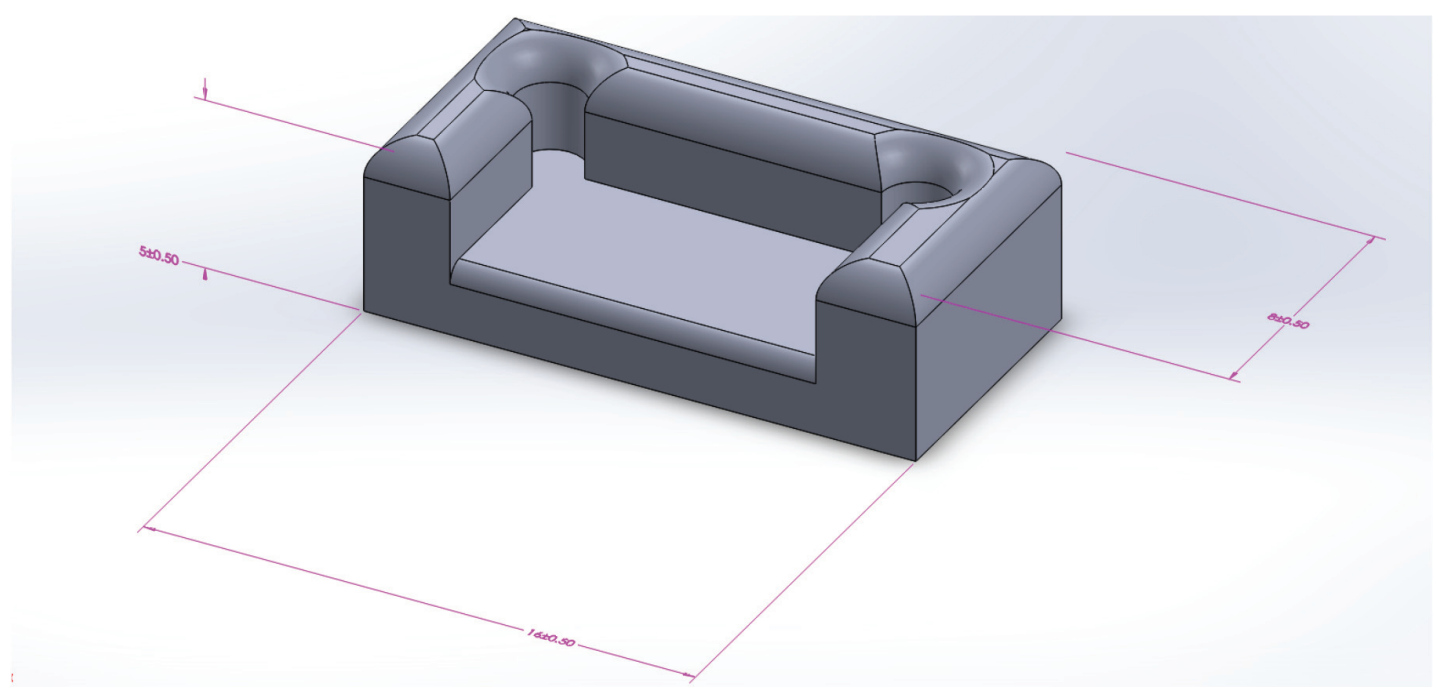

Figure 6. Micro Gap sensor bed as printed in 3D 
the sensor bed. An advantage of the micro-nano-gap capacitor is that the surface area is enhanced by miniature structures $(<440 \mathrm{~nm})$. Because the DUT sensor has small structures, it is sensitive to friction caused by handling. The micro-structure is fragile to strain and touching of the sensor area because the structure is etched under angles, making it possible for the polymer sensing layer to reach all areas. The delicacy of the structure leads to a risk of short circuits, when handled with too much force, rendering them useless. The force, on the other hand, is needed to get the chip out of the carrier container. When the chip is removed from the container it must be handled with curved tweezers. With this solution the sample can be moved from the container to treatment, and then be fed with a minimum of force into the container. After the experiment, the chip can be removed, after the pogo pins are retracted, by lifting it out of the chip holder with tweezers. 


\section{A2.5. Software Design}

The FDC2212 can be configured using the $\mathrm{I}^{2} \mathrm{C}$ interface described above. After construction of the PCB, the first step required is the configuration of the interface and the design of the function call for writing and reading the register set. First, the $\mathrm{I}^{2} \mathrm{C}$ interface, the serial interface and the processor must be initialized after the power is turned on. In order to read and write to the register set of the FDC, two $\mathrm{I}^{2} \mathrm{C}$ software routines were designed.
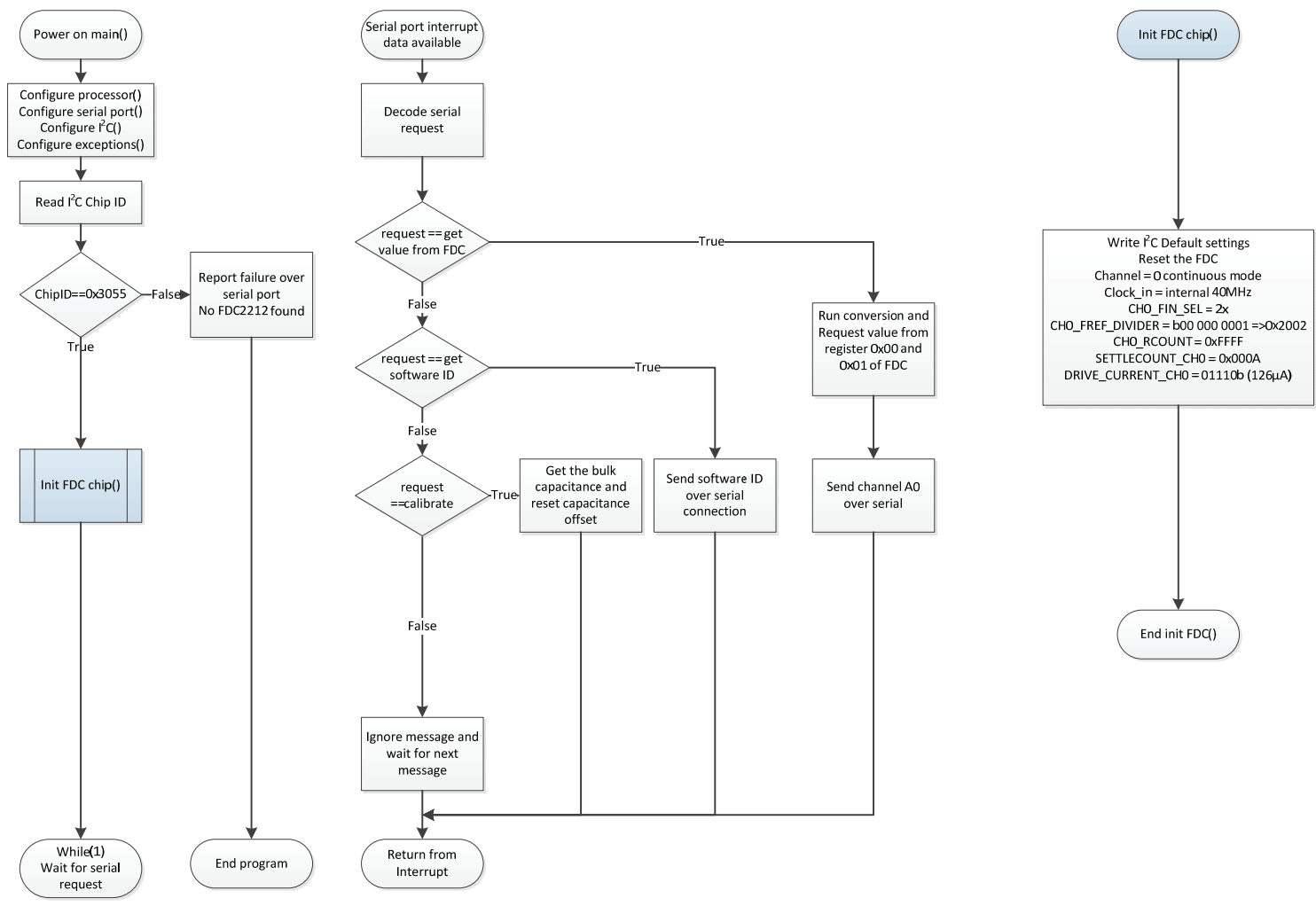

Figure 7. Software flow design for handling the FDC2212 register set

With this software the device can be read and written and error messages that are part of the FDC's architecture can be checked. Figure 7 shows the software design. On the left is the starting software loop where the host MCU, the LPC2148, is initialized. After this the blue block points to the initialization of the FDC chip on the right. Several registers must be supplied with a correct value since this chip can be used for various purposes (see the datasheet of the FDC2212: (Texas_instruments, 2015). 


\section{A3. Measurement Results of the Capacitance Interface}

A series of software acceptance test was designed in order to test the capacitance interface. These tests consisted of:

- Determining the resolution of the sensor (goal $\leq 10 \mathrm{fF}$ )

- Determining the speed of the conversion (goal $\geq 4$ samples per second)

- Determining the noise floor of the sensor (goal <10 fF)

- Determining the linearity of the converter (goal: to be able to linearize the output)

In order to measure this data, test software was developed to obtain a stream of data.

This RS232 output data was logged and fed to Excel for further analysis. The output data of the register of channel A0 is represented in a 28 bit word. This data can be used to calculate the added capacitance or capacitance change. Figure 3 shows the tank circuit of the FDC, consisting of a known capacitance (C) together with the parasitic capacitance of the PCB and inductance (L). Together these components will resonate at certain frequency given by:

$F_{\text {res }}=\frac{1}{2 * \pi * \sqrt{(L * C)}}$

Where $\mathrm{L}$ is the inductance and $\mathrm{C}$ is the combined capacitance. The datasheet suggests an operating frequency between $10 \mathrm{kHz}$ and $10 \mathrm{MHz}$ and used an inductor of $18 \mu \mathrm{H}$ and a capacitance of $33 \mathrm{pF}$ with additional $20 \mathrm{pF}$ as a result of PCB wiring, resulting in a resonating frequency of $5.15 \mathrm{MHz}$. After initialization of the FDC this frequency was verified using an oscilloscope, keeping in mind that the probe adds capacitance and therefore influences the tank frequency. The $40 \mathrm{MHz}$ internal master clock was used for comparison of the result of the output buffer. The output register of channel A0 can now be used to verify this.

$F_{\text {SensorA } 0}=\frac{f_{\text {reference }} * D A T A_{-} R E G A 0}{2^{28}}$

Where $f_{\text {reference }}$ is the internal oscillator frequency of $40 \mathrm{MHz}$ and DATA_REGA0 is the value from the output register. This value provides the current frequency of the tank. 
This frequency can be used to calculate the additional capacitance of the tank when Equation (1) is rewritten to:

$C_{\text {sensor }}=\frac{1}{L *\left(2 * \pi * F_{\text {sensor } A 0}\right)^{2}}-C$

Where $\mathrm{L}$ is the inductance, $\mathrm{F}_{\text {sensor }} \mathrm{A} 0$ is the calculated value of Equation 2 and $\mathrm{C}$ is the calculated combined bare IDE capacitance. When a delta value of the capacitance is required, first the $\mathrm{L}$ and combined $\mathrm{C}$ are measured, and this value is subtracted from any new measurement. Note that because a delta capacitance $(\Delta C)$ value is used, the capacitance variance can also be negative.

The interface is used to measure acetone concentrations, meaning that the absolute capacitance is not an issue but the linearity is and must therefore be measured. This was done by adding a known capacitance to the LC tank and calculating the results. Five capacitors were selected in the range of 1 to $10 \mathrm{pF}$ and measured with a Hameg H8018 LRC meter. The output data of the FDC was fed through a five-tab moving average filter (MAF) to smooth the output. Figure 8 shows the result of these measurements plotting the Hameg results against the FDC.

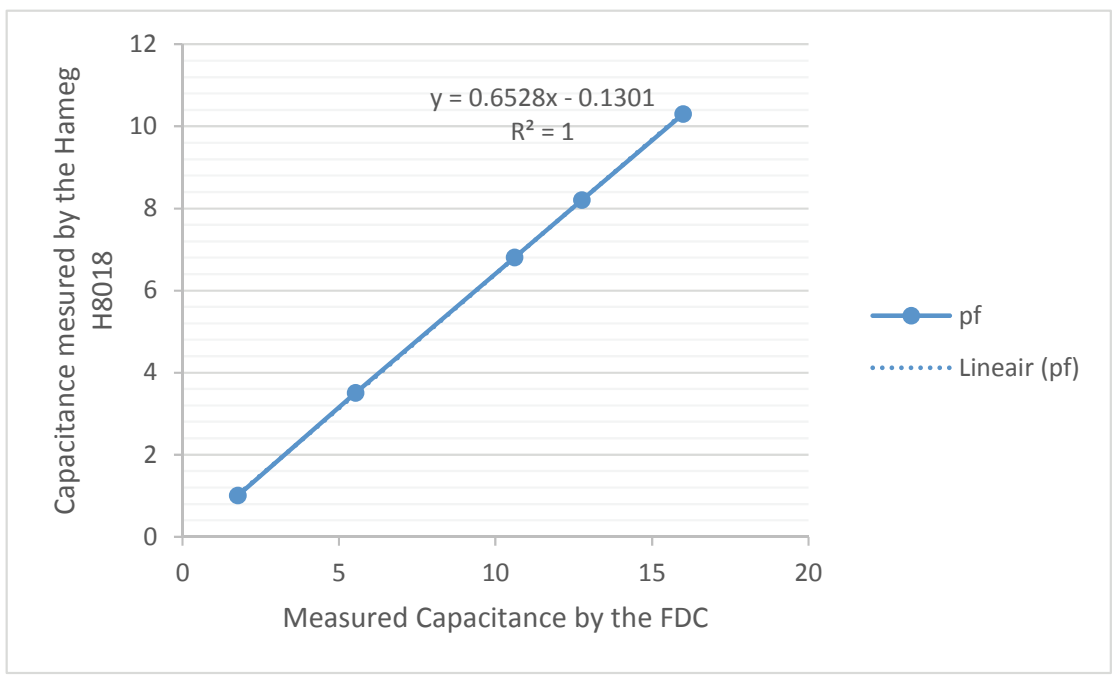

Figure 8. Linearization graph of the FDC2212 against the Hameg H8018 
Here we observe that the standard deviation is equal to one, suggesting that the system does not need linearization. After this, the code was modified to constantly output values on the terminal with a frequency of $4 \mathrm{~Hz}$ while logging the data for further analysis. The data shows that the interval frequency of $4 \mathrm{~Hz}$, well within the minimum conversion speed of $26 \mathrm{~ms}$ per sample, is stable. The FDC can handle faster speeds, up to 100 samples per second (Sps) at the cost of resolution. The FDC is set to maximum resolution in this application and kept at this setting for the rest of the measurements. Then, the FDC2212 was placed in open space (see Figure 5) and samples were collected over a period of 30 seconds. This data was modified using the method described above in order to obtain a value for $\Delta C$. This data was plotted in Figure 9 to estimate the noise figure in open space.

It was observed that during this interval the $\Delta \mathrm{C}$ was a little under $1.3 \mathrm{fF}$, a factor 7.5

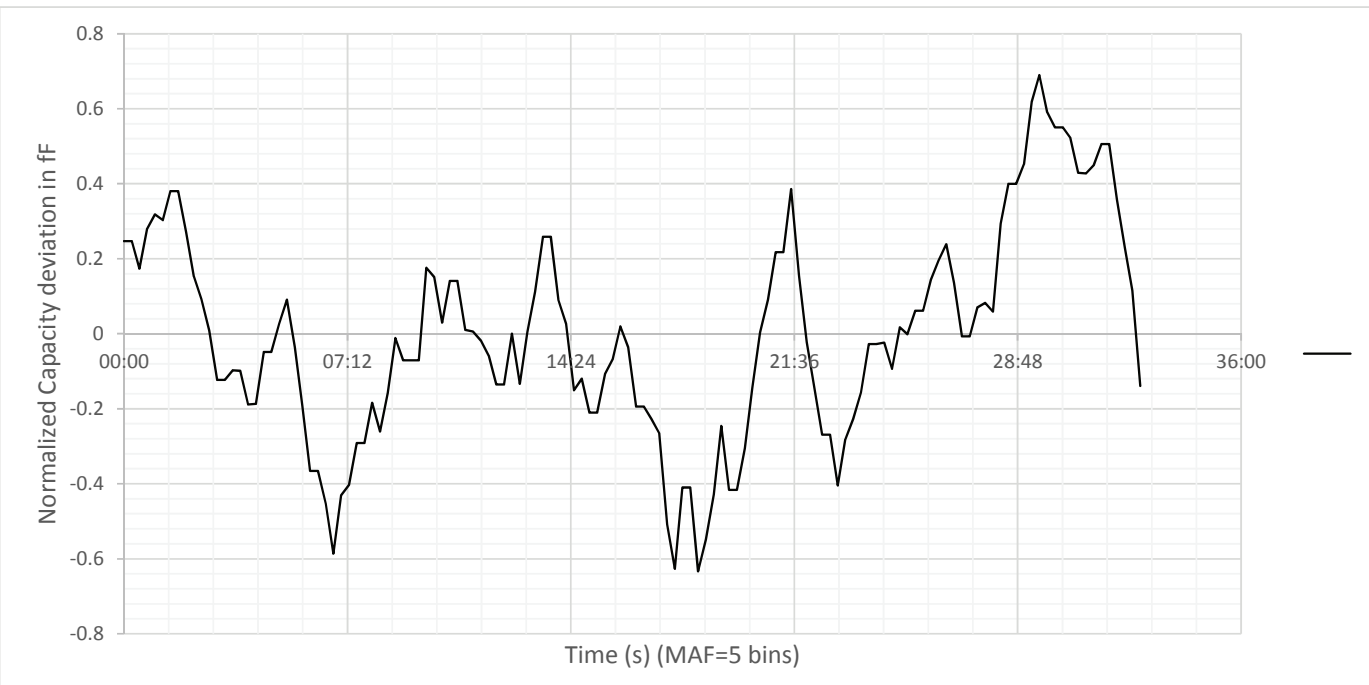

Figure 9. Noise figure of the FDC 2212 in femtofarads

better than the minimum goal of $10 \mathrm{fF}$. The smallest resolution step at the highest tank frequency ( $48 \mathrm{MHz}$ ) is $0.3 \mathrm{fF}$. This minimum resolution would improve with a lower LC oscillator value.

This concludes the measurements on the capacitance interface. At this time this noise figure and its resolution and noise floor are acceptable and this configuration was deemed acceptable for use in the SMS. 


\section{Appendix B}

\section{Calculation of the Differential and common mode voltages}

$$
\begin{aligned}
& \text { Vout }=\operatorname{IC} 5(\mathrm{~min})-\mathrm{IC} 5 \text { (plus) } \quad \text { with R4=R5 } \\
& \mathrm{IC} 5(\min )=\frac{-\mathrm{Vout}+\mathrm{Va}}{2} \text { (1) } \quad \mathrm{IC} 5(\text { plus })=\frac{1}{2} \cdot \mathrm{Vb} \quad \text { (2) } \\
& \mathrm{V} 1=\mathrm{Vcm}+\frac{1}{2} \cdot \mathrm{Vdiff}(3) \quad \mathrm{V} 2=\mathrm{Vcm}-\frac{1}{2} \cdot \mathrm{Vdiff} \\
& \mathrm{Va}=\frac{\mathrm{V} 1-\mathrm{Vm}}{\frac{1}{2} \mathrm{R} 1} \cdot \mathrm{R} 2+\mathrm{V} 1(5) \quad \mathrm{Vb}=\frac{\mathrm{V} 2-\mathrm{Vm}}{\frac{1}{2} \mathrm{R} 1} \cdot \mathrm{R} 3+\mathrm{V} 2 \\
& \mathrm{Vm}=\frac{\mathrm{V} 1-\mathrm{V} 2}{\mathrm{R} 1} \cdot \frac{1}{2} \mathrm{R} 1+\mathrm{V} 2 \\
& \mathrm{Vout}=\mathrm{Vb}-\mathrm{Va} \\
& \text { Vout }=-\frac{\mathrm{R} 1 \cdot \mathrm{V} 1+2 \cdot \mathrm{R} 2 \cdot \mathrm{V} 1-\mathrm{R} 1 \cdot \mathrm{Vb}-2 \cdot \mathrm{R} 2 \cdot \mathrm{Vm}}{\mathrm{R} 1} \text { substitute } \mathrm{Vb} \text { with (5) and Va with (6) } \\
& \text { Vout }=-\frac{\mathrm{R} 1 \cdot \mathrm{V} 1-\mathrm{R} 1 \cdot \mathrm{V} 2+2 \cdot \mathrm{R} 2 \cdot \mathrm{V} 1-2 \cdot \mathrm{R} 3 \cdot \mathrm{V} 2-2 \cdot \mathrm{R} 2 \cdot \mathrm{Vm}+2 \cdot \mathrm{R} 3 \cdot \mathrm{Vm}}{\mathrm{R} 1} \quad \begin{array}{l}
\text { Substitute } \mathrm{Vm} \\
\text { with }(7)
\end{array} \\
& \text { Vout }=-\frac{(\mathrm{V} 1-\mathrm{V} 2) \cdot(\mathrm{R} 1+\mathrm{R} 2+\mathrm{R} 3)}{\mathrm{R} 1} \quad \text { Substitute V1 with (3) } \\
& \text { Vout }=-\frac{\left(\mathrm{Vcm}-\mathrm{V} 2+\frac{\mathrm{Vdiff}}{2}\right) \cdot(\mathrm{R} 1+\mathrm{R} 2+\mathrm{R} 3)}{\mathrm{R} 1} \quad \text { Substitute and V2 with (4) } \\
& \text { Vout }=\frac{\mathrm{Vdiff} \cdot(\mathrm{R} 1+\mathrm{R} 2+\mathrm{R} 3)}{\mathrm{R} 1} \quad \text { Common mode voltage } \mathrm{Vcm} \text { is no longer present }
\end{aligned}
$$


Appendix B 


\section{Summary}

Nanotech-based sensors have been designed to consume less power and to decrease their size, making them suitable for application in low-power wireless sensor networks (WSN) systems. The challenge is not to compromise on the sensor's sensitivity and selectivity towards the target. This thesis studies various options to improve the limits of detection. Usually, sensors are limited in detection range and resolution; they are also restricted by cross sensitivity towards other target agents and other parameters. This cross sensitivity, towards humidity and temperature, for instance, reduces the sensor's detection limit and, therefore, its usability. A Signal Measurement System (SMS) is required to study and measure sensor parameters, such as sensitivity, selectivity and cross sensitivity, and to parameterise the properties of the experimental sensor. This work demonstrates that the design of the SMS is a key element in improving the limits of detection and that a second identical sensor can be used to reduce cross-sensitivity effects.

This work discusses three examples where the limit of detection has been investigated, localised and improved with nanotech-based sensors.

The first example is a nanowire-based hydrogen sensor, in which a second identical sensor is used to compensate for cross sensitivity towards temperature. Paired palladium nanowires were deposited on a silicon chip using an innovative process called deposition and etching under an angle. This sensor presented an excellent response with a large dynamic range of hydrogen concentrations (27 to 12,000 ppm) while being biased with only $50 \mu \mathrm{A}$. The detection-limiting factor in the usage of this type of sensor is the cross sensitivity towards temperature. The properties of the sensor chip were parameterised with two paired nanowires in the SMS that was modified for closed-loop control of a desired hydrogen concentration. Normal cross sensitivity with respect to temperature was found to be $600 \mathrm{ppm} /{ }^{\circ} \mathrm{C}$, limiting this sensor's detection and, therefore, its usage. After analysing the data, a digital-signal process was designed to compensate for the temperature error using an ordinary temperature sensor. This decreased cross sensitivity, however, during rapid temperature changes, the mismatch 
in time constants of these different sensors, temperature and nanowire negatively influenced the limit of detection. This problem was overcome by introducing a second matched nanowire on the same silicon, pacified for hydrogen, to compensate for temperature changes by perfectly matching the time constants. This method also made it possible to measure an early relative response of a hydrogen change within $600 \mathrm{~ms}$, whereas an absolute hydrogen level can be measured within 15 seconds.

The second example was based on a potentiometric-sensor principle, capable of sensing $\mathrm{CO}_{2}$ at room temperature. The absence of a heating element makes is possible to integrate these sensors in a WSN. The rubidium-silver-iodine-based sensor also presented a severe cross sensitivity to temperature. Usually, this type of sensor is combined with a heater to measure at relatively high $\left(>150^{\circ} \mathrm{C}\right)$ and stable temperatures because when the measurement is conducted at room temperature, this temperature dependence negatively influences the limit of detection. To understand the responsible parameters, the SMS was modified to account for the closed-loop control of a targeted $\mathrm{CO}_{2}$ concentration. A custom amplifier and analogue-to-digital converter, which could simultaneously interface with four potentiometric sensors, was designed and integrated into the SMS. The Nernst equation, which relates the voltage output (Electro Motive Force) to the temperature, was used to improve the limits of detection. It is shown that an external temperature sensor can be utilised to compensate for the cross sensitivity of the potentiometric sensor's output signal. However, when the output of two sensors is combined, the improvement in the limit of detection increases. One of the sensors was passivated for $\mathrm{CO}_{2}$, while the others were open for $\mathrm{CO}_{2}$ detection. During a temperature variation of $22^{\circ} \mathrm{C}$, the open and passivated sensors were exposed to an inlet of 4,000 ppm $\mathrm{CO}_{2}$. The limit of sensitivity detection increased by a factor of 4.5 measured over a time period of 22 hours. With potentiometric sensors operating at room temperature, another factor that must be considered is their reduction in ion activity, which reduces the sensors' output voltage over time. A six-month measurement was taken, which proved that a relatively simple exponential equation, derived from fitted data and combined with the mathematical integration of measurement data during the sensors' operational life, could be used to further improve the limits of detection.

The third example is an Inter-Digitated Electrode (IDE) sensor, capable of selective sensing through the coating of an active layer, which serves as a dielectric. Although this method is widely used, it is restricted by the amount of change in the dielectric relative 
to the bare IDE capacity of the IDE. This restriction could mean that a highly selective synthesized coating with a low impact on the dielectric change cannot be significantly measured because of a poor signal-to-noise ratio. This study indicates that restrictions are caused by three factors. The first factor is the measurement setup combined with the capacitance measurement system. Measurements indicate that 1 metre of cabling in the measurement setup restricts the measurement resolution to $31 \mathrm{f} \mathrm{F} /{ }^{\circ} \mathrm{C}$. This leads to the conclusion that measurements must be taken as close as possible to the experimental IDE to reduce the influence of cabling to zero. The second factor is the resolution restriction of the measurement device itself. The outcome of the first factor led to the design, build and verification of a custom measurement-capacitance interface, based on a semiconductor from Texas Instruments, which offers a $0.3 \mathrm{fF}$ resolution with $168 \mathrm{~dB}$ dynamic range and would fit inside the SMS. This capacitance-measurement interface was integrated into the SMS and connected to a Micro Controller Unit (MCU), which was programmed to deliver new readout values on host PC command. The interface was verified using graphene oxide (GO), which has been well documented, as an active coating on a (36 pF) IDE. The results illustrate an improvement in measurement resolution from picofarads to femtofarads (a factor of 1,000) compared to other work with equivalent results. The third factor is the area density of usable IDE space on the silicon. Increasing the IDE surface density can also improve the signal-to-noise ratio since a small bare IDE capacity and, therefore, the measurement of an even smaller $\Delta \mathrm{C}$ change determines the detection limit. This positive effect was demonstrated by experiments on two polymer-based IDE sensors with micro- (8 pF) and nano- (135 pF) scale features resulting in a higher IDE capacitance. Both IDE sensors were coated with an acetone-sensitive layer of poly (4-vinylphenol) (PVPH 10\%) and, after appropriate SMS modifications, were tested with acetone concentrations up to 1,000ppm. Both the micro- and nano-density sensors showed a relative change of $0.2 \%$ with respect to the bare IDE capacitance, although the signal-to-noise factor and, therefore, the limit of detection increased by a factor of 12 with higher surface density.

This threefold improvement on capacitive-based IDE sensors enables the application of more selective active layers with a lesser sensitivity towards a dielectric change herewith further improving the limits of detection in sensor systems. 


\section{Acknowledgements}

It was foretold that contributing to science and society in doing research is not easy. Time is, sure enough, always of the essence. Fortunately, I have enjoyed many supporters, shared ideas and took advice, and for that I am grateful. During this PhD project I have had countless discussions with many peers about how to gather data that was needed, how to interpret the findings and how to improve the concept or, even more important, the decision to abandon a chosen solution and start all over.

First of all, I would like to thank my wife Gerdien Dijkstra for never doubting me and supporting me all the way, and my daughters, Ling and Yun, for always making me smile. In general, I am thankful for my friends, family and colleagues for believing in me and in this ambitious project.

I would like to thank my promotor and supervisor Cees van Rijn for his guidance and time, the many pointers he gave in scientific writing, and the many discussions I have enjoyed during the PhD project. I would like to thank the department Laboratory of Organic Chemistry for support and advice. Furthermore I would like to thank Erik Puik for his many efforts of improving the writing and discussing the measurement setup and results. I would especially would like to thank him for his advice and support on the journal articles and, of course for his role as paranymph. I would like to thank Hien Tong for providing me with experimental chips and for the many discussions we have had on how to perform measurements on the often fragile and ESD sensitive devices. I would like to thank Michiel Scager for moral support over the past years and for honouring me in the role of paranymph. I would like to thank Albert van Duin and Klaas Dijkstra from SRON for letting me use their wedge bonder and other equipment and advising me on the palladium chips. Furthermore, my thanks goes to Elly Geurtsen for administrative work, Tin Doan, Ai Nguyen, Anke Kuijk, Han Zuilhof, Bart Bozon, Ton Marcellis, and Jacob Baggerman for interesting discussions and support, and Janny Bakker - de Leeuw for her warm presence and assistance. Also, please accept my humble apology in case I have forgotten to mention anyone.

Franc 


\section{About the author}

Franc van der Bent was born on April 28, 1971 in Voorhout, the Netherlands. He received the B.S. degree from the Department of Electronics and Information Engineering, University of applied Science and Technology, and the MSc degree in the field of IPD. From 1996 until 2000, he worked at the University Medical Centre in Utrecht as an electronics and embedded software engineer. From 2000 on accepted a teaching position at HU University of applied science in Utrecht. From 2009 he started as a part time Ph.D student at the WUR Wageningen University. He is now a senior lecturer at the HU University of applied science, Utrecht at the electrical engineering and design group. 


\section{List of publications}

\section{Conference Papers}

van der Bent, J. F., Puik, E. \& van Rijn, C. J. M. (2010, July6-9). An approach to utilize a grid of EMF potentiometric CO2 sensors in a low power wireless sensor network. Paper presented at the APCOT Australia-CINN, Perth,p288.

van der Bent, J. F., van Rijn, C. J. M. \& Puik, E. (2010). Ultra low power temperature compensation method for palladium nanowire grid. Procedia Engineering, 5, 184187.

Journal Papers

Bent, J. F. van der, Puik, E. C. N., Tong, H. D. \& Rijn, C. J. M. van. (2015). Improving the limits of detection in potentiometric sensors. Measurement Science and Technology, 26(12), 125104.

Doan, Tin C. D., Ramaneti, Rajesh, Baggerman, Jacob, van der Bent, J. Franc, Marcelis, Antonius T. M., Tong, Hien D. \& van Rijn, Cees J. M. (2012). Carbon dioxide sensing with sulfonated polyaniline. Sensors and Actuators B: Chemical, 168(0), 123-130.

doi: http://dx.doi.org/10.1016/j.snb.2012.03.065

van der Bent, J. F., Puik, E., Tong, H. D. \& van Rijn, C. J. M. (2015). Temperature balanced hydrogen sensor system with coupled palladium nanowires. Sensors and Actuators A: Physical, 226(0), 98-106.

doi: http://dx.doi.org/10.1016/j.sna.2015.01.037

Submitted Journal Papers

Franc J.F. van der Bent, Quyen Nguyen Minh, Hien D. Tong, and Cees J.M. van Rijn. Improving the Limits of Detection in capacitive sensors systems. Measurement Science and Technology

Quyen Nguyen Minh, Anke Kuijk, Sidharam P. Pujari, Franc van der Bent, Hien D. Tong, Han Zuilhof, and Cees J.M. van Rijn. Preparation and Gas Sensing Properties of Nanocomposite Polymers on Micro-Interdigitated Electrodes for Detection of Volatile Organic Compounds at Room Temperature. Sensors and Actuators B: Chemical, Elsevier

Journal Papers in preparation

Quyen Nguyen Minh, Anke Kuijk, Hien D. Tong, Franc van de Bent, Pepijn Beekman, Han Zuilhof, and Cees J.M. van Rijn. Excellent Gas Sensing Performance at Room 
Temperature of Two-dimensional Nanogap Interdigitated Electrodes for the Detection of Acetone at Low Concentration. ACS Sensors 


\section{Overview of completed training activities}

Courses

2016 Scrum Master, Xebia

2014 Presenting to the point

2014 Cursus Didactisch Handelen

2013 Scientific Writing, Inhuis cursus voor promovendi, TU delft

2009 Research methods and Techniques HU

Conferences

Eurosensors, Lausanne 2009

Technologie stichting STW, 2009

APCOT. Perth 2010

MINACNED conference, Eindhoven 2010

Eurosensors, Linz Austria 2010

DEVLAB Development laboratories, 2010

Colloquium presentation, Wageningen 2010

Nanosens Nanosensors for Industrial Applications, Austria 2010

DEVLAB Development laboratories, Eindhoven 2011

User Interface Design seminair Den Bosch 2014

Sense of contact seminair Den Bosch 2015 


\section{Optionals}

Active participation (meetings and presentations in EU- projects)

Technologie stichting STW Eindhoven 2009-2011

Artemis SMART and the European Collaborative Project SiNAPS 2009-2015 
The research described in this thesis was financially supported by the HU University of Applied Sciences Utrecht.

Thesis layout: $\quad$ J.F. van der Bent

Cover page: SEM image of a dual $60 \mathrm{~nm}$ width palladium nano wire

Printed by Digiforce print and logistics, Vianen, The Netherlands 